\title{
Vascular Flora and Endemism of Ormanüstü Planning Unit (Maçka-Trabzon), Turkey
}

\author{
Alper UZUN ${ }^{1 *} \mathbb{E}$, Salih TERZİĞLU ${ }^{2} \bullet$ \\ ${ }^{1}$ Kahramanmaraş Sütçü İmam University, Faculty of Forestry, Department of Forest Botany, \\ Kahramanmaraş, TURKEY \\ ${ }^{2}$ Karadeniz Technical University, Faculty of Forestry, Department of Forest Botany, Trabzon, TURKEY \\ *Corresponding Author: auzun@ksu.edu.tr
}

Received Date: 04.11.2020

Accepted Date: 22.03.2021

\section{Abstract}

Aim of study: Aim was to determine the vascular flora of the Ormanüstü Planning Unit (Karadeniz Technical University Education-Research and Application Forest).

Area of study: The research area is under the influence of the Euro-Siberian phytogeographical region and is represented with a high rate $(44 \%, 288$ taxa). Irano-Turanian and Mediterranean elements are represented by $26(4 \%)$ and 22 taxa (3\%), respectively. Totally 320 taxa (49\%) are multi-regional or of unknown origin. In addition, 33 endemic (5\%), 12 rare (1.8\%), 5 alien $(0.8 \%)$ and 17 cosmopolitan $(2.6 \%)$ taxa were identified.

Material and methods: The entire study area was visited periodically and plant samples were collected from 166 sample plots $\left(1000 \mathrm{~m}^{2}\right)$, and then they were pressed with wooden frames. Approximately 1800 vascular plant specimens were collected between the years 2005 and 2008 to form the floristic list.

Main results: Studies revealed the presence of 656 taxa (585 species, 62 subspecies and 9 varieties) belonging to 95 families and 354 genera. Of these, 22 taxa (3.4\%) are ferns and $634(96.6 \%)$ are seed plants. The best-represented families are as follows; Asteraceae (75), Poaceae (48) and Fabaceae (41). According to richest genera; Campanula (14), Carex (10), Alchemilla (9) and Geranium (9) have the greatest number of taxa.

Highlights: It was concluded that the study area is important in terms of plant diversity. The discovery of a new species from this area, described by us and published in 2009 (Astragalus ansinii Uzun, Terzioğlu \& Pal.-Uzun), supports this fact.

Keywords: Vascular flora, Endemism, Colchis, Maçka, Turkey

\section{Ormanüstü Planlama Birimi Vasküler Florası (Maçka-Trabzon), Türkiye}

Öz

Çalışmanin amact: Bu çalı̧̧mada Ormanüstü Planlama Birimi'nin (Karadeniz Teknik Üniversitesi Eğitim-Araştırma ve Uygulama Ormanı) vasküler florasını belirlemek amaçlanmıştır.

Çalışma alanı: Avrupa-Sibirya fitocoğrafik bölgesinin etkisi altındadır ve yüksek bir oranla (\% 44, 288 takson) temsil edilmektedir. İran-Turan ve Akdeniz elementleri sirasıla 26 (\% 3.96) ve 22 takson (\% 3.35) ile temsil edilmektedir. Toplam 320 takson (\% 49) çok bölgeli veya bilinmeyen kökenlidir. Ayrıca 33 endemik (\% 5), 12 nadir (\% 1.8), 5 yabancı yurtlu (\% 0.8) ve 17 kozmopolit (\% 2.6) takson saptanmıştır.

Materyal ve yöntem: Çalışma alanının tamamı periyodik olarak ziyaret edilmiş ve 166 örnek parselden $\left(1000 \mathrm{~m}^{2}\right)$ bitki örnekleri toplanmış ve ardından preslenmiştir. 2005-2008 yılları arasında yaklaşı 1800 damarlı bitki örneği toplanarak floristik liste oluşturulmuştur.

Temel sonuçlar: Çalışmalar sonucunda, 95 familyaya ve 354 cinse ait 656 vasküler bitki taksonu (585 tür, 62 alttür ve 9 varyete) tespit edilmiştir. Bunlardan 22 takson (\% 3.4) eğrelti otu ve 634 (\% 96.6) takson ise tohumlu bitkidir. En iyi temsil edilen familyalar şunlardır; Asteraceae (75), Poaceae (48) ve Fabaceae (41). En fazla takson sayısina sahip cinsler ise; Campanula (14), Carex (10), Alchemilla (9) ve Geranium (9)'dur.

Araştırma vurguları: Çalışma alanının bitki çeşitliliği açısından önemli olduğu sonucuna varılmıştır. $\mathrm{Bu}$ alandan bizim tarafımızdan tanımlanan ve 2009 yılında yayımlanan yeni bir türün keşfedilmesi (Astragalus ansinii Uzun, Terzioğlu ve Pal.-Uzun) bu gerçeği desteklemektedir.

Anahtar Kelimeler: Vasküler flora, Endemizm, Kolşik, Maçka, Türkiye 


\section{Introduction}

Flora studies constitute the main framework of biodiversity studies. Detailed flora studies in different habitat types are needed in revealing the plant biodiversity existing in the world on a global scale (Funk, 2006).

This study was carried out to determine the vascular flora and endemism of the Ormanüstü Forest Planning Unit located within the borders of Maçka Forest Enterprise of Trabzon Forestry Regional Directorate (Trabzon / Turkey) (Figure 1). This unit was defined as the education-research area for the scientific studies of the Karadeniz Technical University Faculty of Forestry (KTU) in 1985 and has been used as the education-research and application forest for the last forty years. The forest planning and management operations of this area are carried out by Maçka Forest Enterprise and KTU together (Yolasığmaz, 1998).

In addition to being an education-research area, the following factors were also taken into account in the selection of this area: (1) no detailed floristic study was carried out in the study area except for the limited individual plant collection directed at some species (Anşin, 1982; Hayırlığlu-Ayaz, 1997; Coşkunçelebi, 2001) and bryophyte flora (Erata et al., 2020), (2) the idea that the area could host different plant richness due to its diversity of forest stand types, elevation zones and four aspects of the terrain (OGM, 2006), (3) possibility of integrating floristic data into existing studies (Yolasığmaz, 1998; Altun et al., 2002) (especially for forest management and silviculture) or combining with future studies for other disciplines.

\section{Material and Method \\ Study Area}

The study area lies within the boundaries of the Eastern Black Sea Region, Maçka district in Trabzon province, and is located

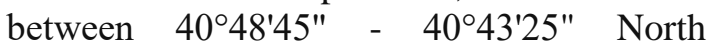

latitudes and 39 36'41" - 39²8'39" East longitudes (Figure 1). This area is totally 5990 ha and surrounded by Yeşiltepe village to the north, Maçka district centre to the east, Çatak village to the west, and Hamsiköy village to the south. Kuzu Korusu Hill is the highest point of the area with $2280 \mathrm{~m}$ a.s.l. The eastern border of the study area is surrounded by Naldöken Hill (1179 m), Kale Tepe (1096 m) and Maçka district centre, and the western border is surrounded by Paparza, Çamlıdüz and Maçka streams. The study area, which has an average elevation of $1330 \mathrm{~m}$ (380-2280 m), has the characteristics of a mountainous terrain in terms of geomorphology. The northwest and the southeast are the main aspects of the study area. Vazelon monastery, built in $270 \mathrm{AD}$ (Çavuş, 2016), is located within the study area (Figure 2D). This region attracts tourists from the world, as it is a spiritual tourism point. Because of its ecological integrity, the study area is located in the Caucasus Ecoregion where the biodiversity is under many threats (Olson et al., 2001; Olson \& Dinerstein, 2002). The main threats in the region are the fragmentation and transformation of habitats due to agricultural activities (mostly hazelnut and tea plantations) (Palabaş-Uzun et al., 2012).

This region is one of the 200 ecological regions in the world that are designated as conservation targets by the World Wildlife Fund (WWF, 2009). The study area is also inside the Caucasus Biodiversity Hotspot. This hotspot region was defined by the International Conservation Organization (CI) and considered to be one of the 34 richest and the most threatened hotspot regions of the world (Myers et al., 2000). In addition, the study area is located within one of the 305 important nature area of Turkey named as Eastern Black Sea Mountains (coded DK005) (Eken et al., 2006). 

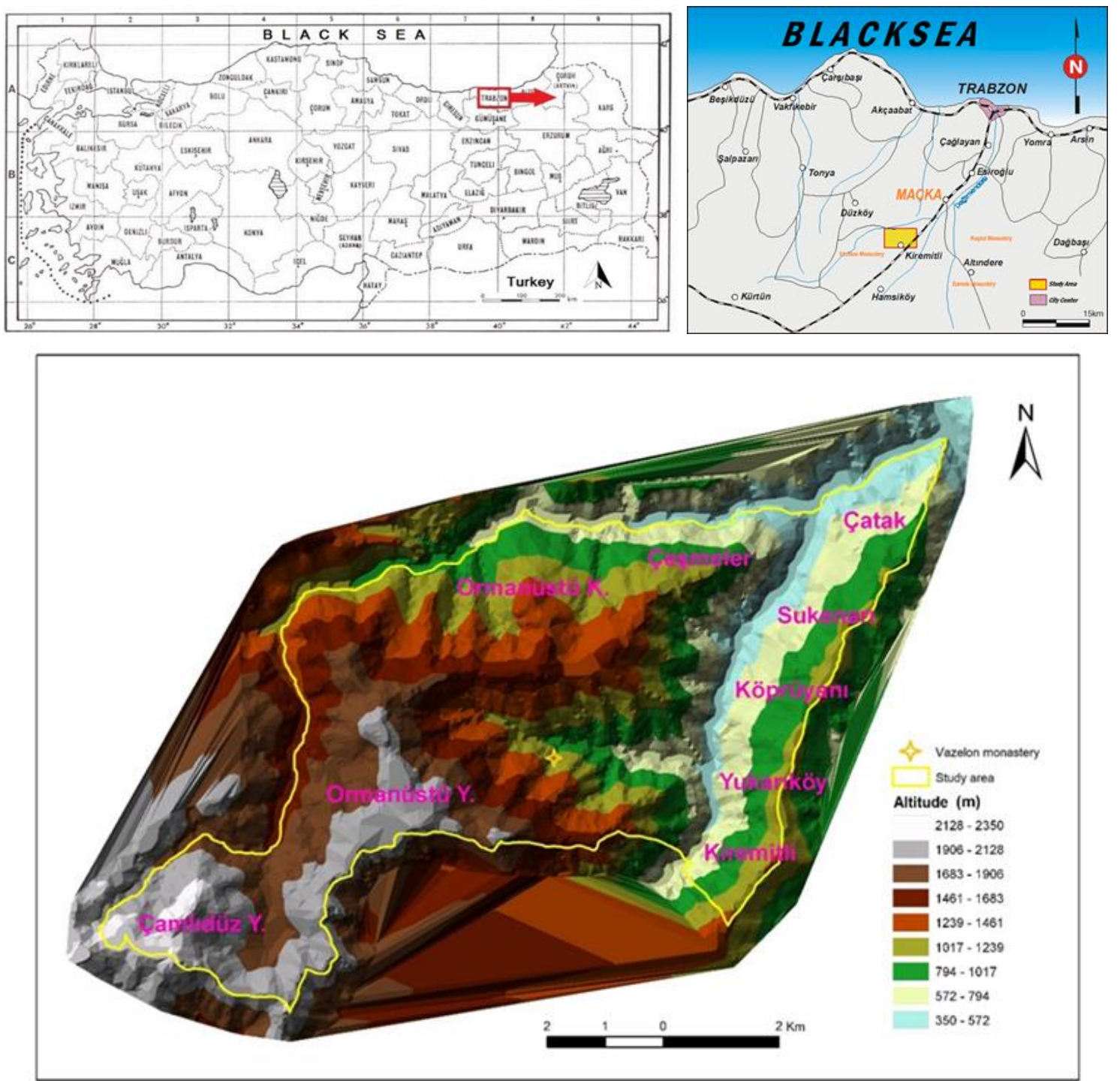

Figure 1. Map and digital elevation model of the study area

\section{Vegetation}

Of the study area; $71 \%$ is state forest and $29 \%$ is pasture, agricultural fields and settlements. Of the forest area, $48 \%$ (2883 ha) productive forest, $15 \%$ ( $868 \mathrm{ha}$ ) degraded forest, $6 \%$ (402 ha) soil suitable for forest and
2\% (126 ha) afforestation area (Figure 2). (OGM, 2006). The study area is particularly diverse in terms of habitats (coniferous and deciduous forest types, forest openings, stony/rocky areas, wet habitats on the edge of the streams and also meadows and pastures). 


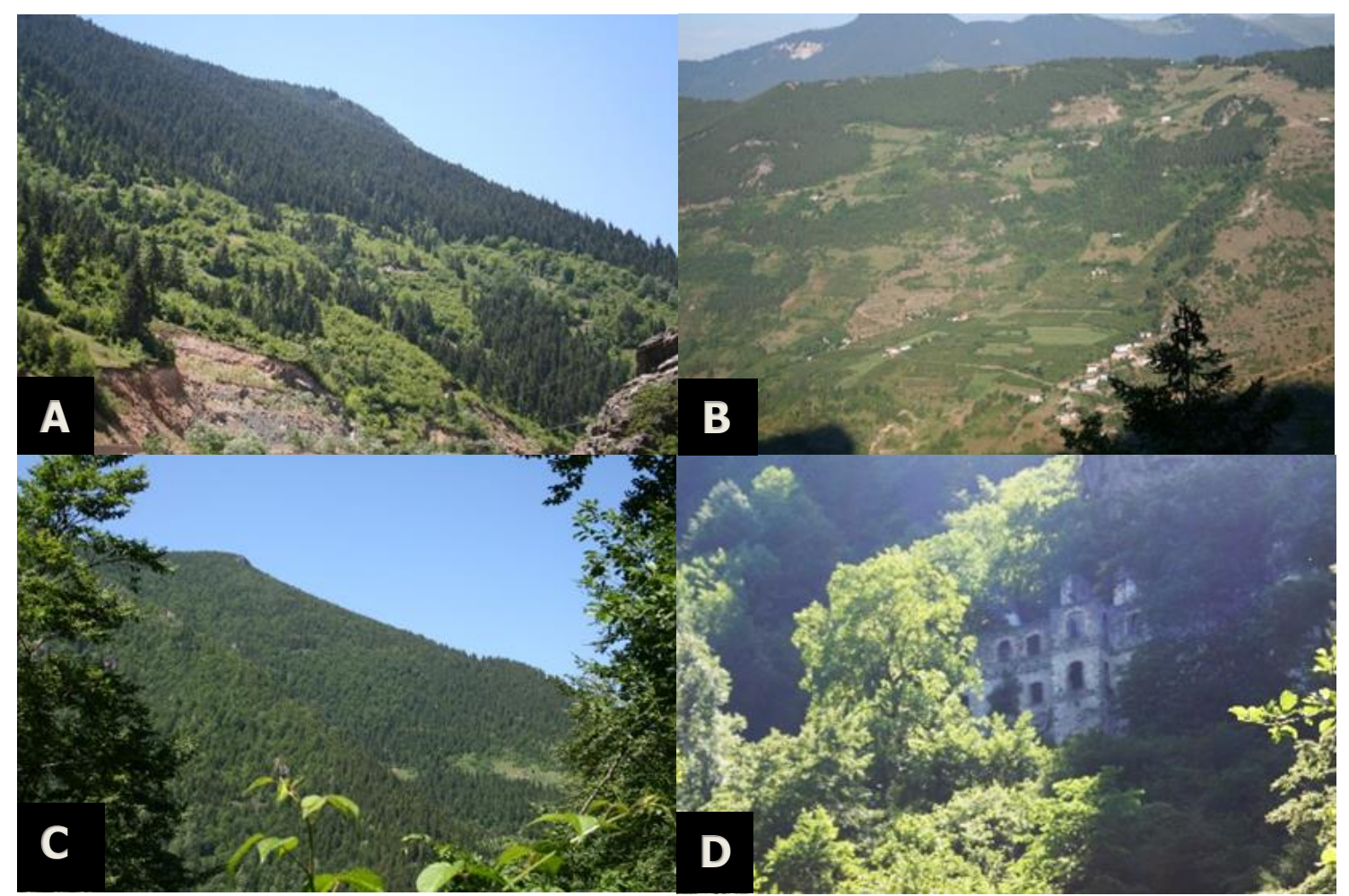

Figure 2. General views from the study area. A: Ormanüstü village with pure coniferous and mixed broadleaved forest. B: Çatak village with degraded forests and agricultural fields. C: Köprüyanı village with pure Oriental spruce (Picea orientalis) forest. D: Vazelon monastery ruins and its surroundings with deciduous broad-leaved Oriental beech (Fagus orientalis) forest.

\section{Geology}

The study area is located in the northern zone of the Eastern Pontides and four different geological formations are exposed in the field. These are from older to younger; Berdiga, Çatak, Kızılkaya and Çağlayan formations (Pelin, 1977). The units are mostly composed of alkaline volcanic, volcanoclastic and sedimentary rocks. Among which; Çatak formation is surfaced in very large areas in Çatak locality along Değirmendere Valley in the southeast of Maçka District. The formation consists of tuff, basaltic and andesitic volcanic rocks, pyroclasts and intermediate additives as, sandstone, claystone, marl (clayey limestone) and the burgundy-colored limestones (Güven, 1993).

\section{Climate Characteristics}

According to Erinç's (1969) climate classification, the study area enters mainly to the Black Sea climate region among four main climate types. However, the "very high precipitation", which is the climatic feature of this climate zone, is not available in all parts of the study area, especially in the southern parts. In this context, the study area shows a characteristic between the Eastern Black Sea and the Central Black Sea climate types. In these areas; mild winters, hot and very rainy summer season are the characteristics of the climate (Akman, 1990). The average annual temperature was $14.7^{\circ} \mathrm{C}$ according to the longterm meteorological data observations (in a period of 1927-2019) by Trabzon Meteorological Station (GDM, 2021). The highest monthly average temperature was observed in August $\left(26.5^{\circ} \mathrm{C}\right)$ and the lowest average temperature was observed in February $\left(4.3^{\circ} \mathrm{C}\right)$. The total average annual rainfall was $829.6 \mathrm{~mm}$, monthly highest in Sept. $(115.4 \mathrm{~mm})$, while the lowest in July (35.6 $\mathrm{mm})$. According to the evaluations made, it is relatively dry from mid-June to the end of August, while other months are quite rainy. Therefore, it can be said that it is under the influence of Subméditerranéen according to Bagnouls \& Gaussen (1957) method (Figure 3). 


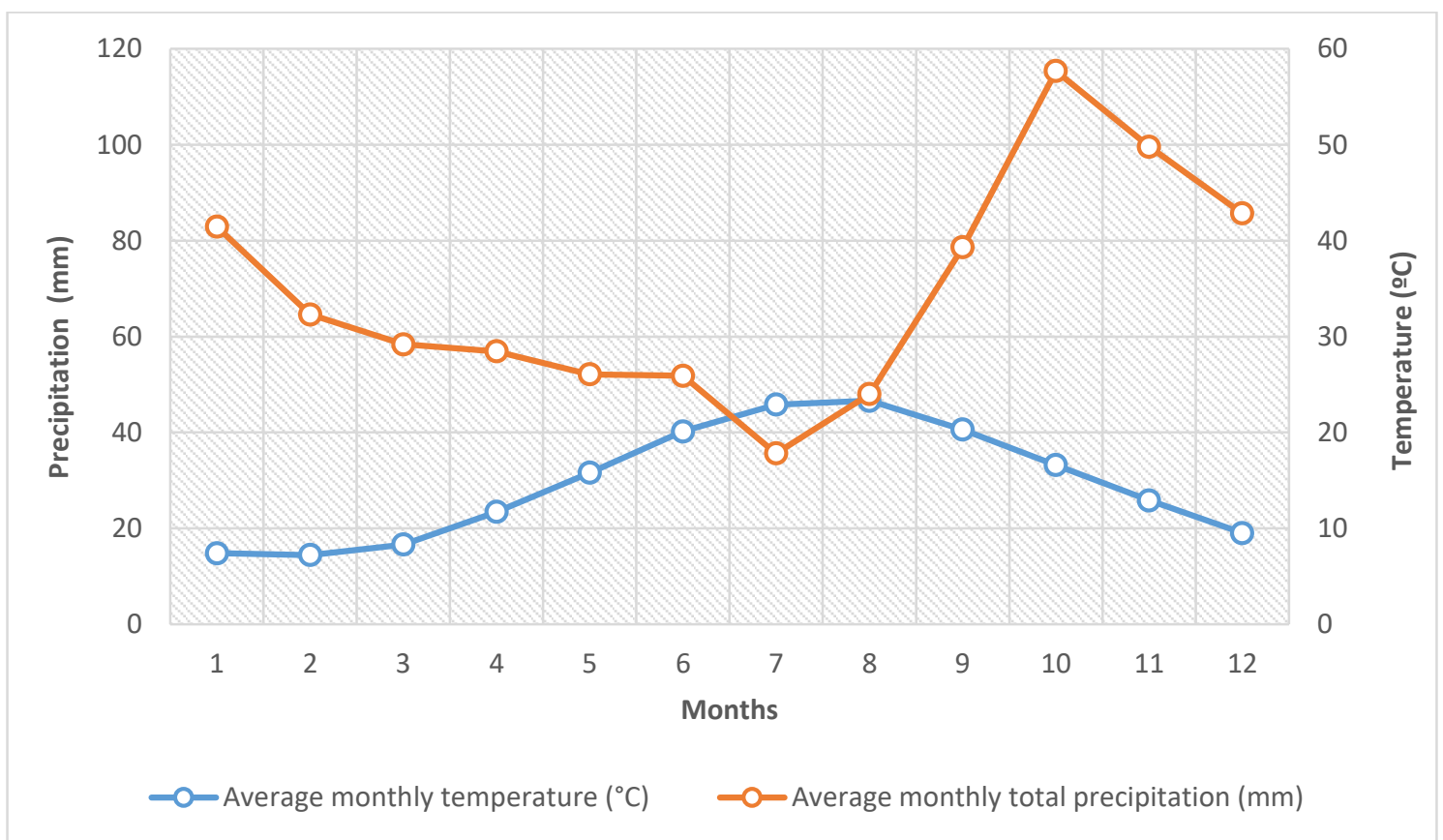

Figure 3. Climatic diagram according to Bagnouls and Gaussen (1957) (Trabzon Meteorological Station, period of 1927-2019)

\section{Soil Characteristics}

The lands of the Eastern Black Sea Region, including the study area, are in the "Podzolic Soil" group among the eight major soil groups in the region (Topraksu Genel Müdürlüğü, 1981). Öztan (1980) explains the soil change in Değirmendere basin of the Eastern Black Sea Region as follows; dominant red-yellow Podzolic soils between sea level and $750 \mathrm{~m}$ altitude, dominant gray-brown Podzolic soils at altitudes of 750-1750 m, and dominant high mountain-meadow soils above $1750 \mathrm{~m}$. According to another study carried out in Trabzon region, neutral and basic magmatic bedrock or on the limestone pseudomacchie soils develop a mull type of humus, these soils are usually in clay loam and slime texture, the reaction of limited acidic, soil washing degree is limited, cation exchange capacity is moderate or high, as reported by Irmak (1974).

\section{Flora Studies}

Materials of the study consisted of collected vascular plant specimens, standard topographic maps with a scale of $1 / 25000$ and stand types map of the region's Forest Management Plan. These stands were used to obtain the digital terrain model of the study area.
Approximately 1800 vascular plant specimens were collected with their roots, stems, leaves, flowers and/or fruits between the years 2005 and 2008 to form the floristic list. A satisfactory material is a sample from a large population with normal habitus with fully matured flowers and ripe fruits. These characteristics are important in terms of full life conditions and changing characteristics of the taxa (Yaltırık \& Efe, 1996; Özer et al., 1998). These plant specimens were dried in accordance with standard herbarium techniques and made ready for identification. After identifications, plant samples were placed at KATO Herbarium (Karadeniz Technical University, Faculty of Forestry) with at least one duplicate sample (Thiers, 2020).

The entire study area was visited periodically and plant samples were collected from 166 sample plots $\left(1000 \mathrm{~m}^{2}\right)$, and then they were pressed with wooden frames. Plants collected around the sample plots were also added to the floristic list. Many plant samples from different habitat types and elevation levels were collected even if they belong to the same species. As it is known, although plant species have the same phenotype, they 
may show systematically important variations according to elevation or habitat types. The identification of these plants with these characteristics is important in terms of completing the floristic study, revealing the variations and determining the taxonomic richness. A label indicating the place where the plant was collected (name of the province, district, neighbourhood, village or plateau), habitat type (forest, rocky place, meadow, swamp etc.), collection date, altitude and collector ID were written on a label for each plant samples.

\section{Floristic List}

Main flora reference of the country "Flora of Turkey and The East Aegean Islands", was used for identification of plant specimens (Davis 1965-1985; Davis et al., 1988; Güner et al., 2000). Following references were also consulted: Flora Orientalis (Boissier, 186788), Flora USSR (Komarov, 1934-78), Flora Europaea (Tutin et al., 1964-80 \& 1993) and Flora Iranica (Rechinger, 1965-1977), illustrated plant atlases such as Bonnier (1912-34), Phillips (1994), Fitter et al. (2000), Hegi et al. (1977); a glossary of terminology (Baytop, 1998), taxonomic revisions, updated check-lists and typifications (HayırlığluAyaz, 1997; Speta, 1998; Coşkunçelebi, 2001; Coşkunçelebi \& Beyazoğlu, 2003; Güner, 2006; Thulin et al., 2009; Zidorn 2012; Frajman et al., 2013; Wang, 2014; Banasiak et al., 2016; Moore \& Dillenberger, 2017; Bartolucci et al., 2018; Farjon, 2018; Gutiérrez-Larruscain et al., 2019; Akçiçek 2020; WCSP, 2020), and recent molecular phylogenetic studies (PPG I, 2016; Wei et al., 2017, Roma-Marzio et al., 2017). We also investigated pertinent flora studies such as Palabaş-Uzun \& Anşin (2006), Palabaş-Uzun \& Terzioğlu (2019).

Some unidentified plant specimens were compared with those in the herbaria KATO, ANK and GAZI. In addition to the main flora works, many other studies on current taxonomic measurements or arrangements related to some genera, species, subspecies and varieties have been examined and evaluated (Kaya, 2003; Coşkunçelebi \& Beyazoğlu， 2001; Hayırlığlu-Ayaz \&
Beyazoğlu, 2002; Hayırlığlu-Ayaz \& Kalheber, 2002; Coşkunçelebi, 2003; Kučera et al., 2006; Al-Shehbaz et al., 2007; Bräutigam \& Greuter, 2007; Karaer \& Celep, 2008).

As stated by Anşin (1980, 1983), making a list containing many features of taxa determined to reveal flora is useful for any research related to conservation, technical forestry and ecology in the region. For this purpose, the categories of taxa identified are given in a systematic list, while taxa belonging to ferns and fern allies are given according to PPG I (2016), taxa belonging to Gymnosperms are classified according to Farjon (1998; 2nd ed. 2001) and Angiosperms (subsections, classes, orders and families) are listed according to APG IV 2016 system (Chase et al., 2016) with the exception of Dipsacales (Reveal, 2011), Caryophyllales (Hernandez-Ledesma et al., 2015) and Boraginales (Luebert et al., 2016). The genera and taxa order in Turkish flora was applied for the present flora list. The author citations of plant names were standardized following the Rec. 46A Note 1 of the ICN (McNeill et al., 2012), i.e. according to IPNI (2012 onwards). Furthermore, the common Turkish or local names of the taxa were given according to Baytop (1994). The floristic list was updated using the recent checklist of the Turkish flora (Güner et al., 2012) and the taxonomic databases such as Euro+Med. Plant Base (2006 onwards) and The Plant List (2013 onwards). The accepted names were given with their synonyms or basionyms that in bracket.

In the floristic list, scientific names were given together with taxonomic categories. Family, genus, species, subspecies and varieties were numbered in order for convenience. In addition, phytogeographical regions of known ones and KATO numbers were added to the floristic list. Also, cosmopolitan, alien (exotic) and parasitic taxa were specified as the characteristics of the study area.

Since the study area stayed within the A7 square according to Davis's (1965) grid system and in the Maçka district of Trabzon province, these expressions were not included in the floristic list in order to avoid repetition. 
To shed light on nature conservation studies, endemic and rare taxa are listed together with IUCN threat categories (Ekim et al., 2000; IUCN, 2001). In addition, the species in the BERN and CITES annexes are specified for the study area (Inskipp \& Gillett, 2005; BERN Convention, 1979). It is also very important to note the field observations and experiences in order to form a basis for conservation studies (Lambeck, 1997). For this purpose, the field observations supported by the density information of the endemic and rare taxa were given in accordance with the scale given below (Table 1).

Table 1 . Scale of estimated population density

\begin{tabular}{cl}
\hline Dense & \\
\hline DW: & Dense-widespread population \\
\hline DP: & Dense-partially distributed population \\
\hline DL: & Dense-localized population \\
\hline Sparse & \\
\hline SW: & Sparse-widespread population \\
\hline SP: & Sparse-partially distributed population \\
\hline SL: & Sparse-localized population \\
\hline
\end{tabular}

UTM coordinates (based on the WGS84 Datum) of the endemic and rare taxa were gathered using GPS device (Geographic positioning system) in the field. The obtained location points were transferred to ArcView module of Arc / Info software and shown in the digital terrain model created by ArcView (Figure 7). In this way, it is aimed to have more information about the structure of the terrain where endemic taxa are located. As well, it was predicted that this information could constitute a basis for the creation of species maps.

\section{Results}

Numerical Analysis of the Flora

A total of 656 vascular plant taxa (585 species, 62 subspecies and 9 varieties) belonging to 95 families and 354 genera in Pteridophyta and Spermatophyta sections were identified in the study area. Of these, Pteridophyta has 22 taxa (3.4\%) belonging to 10 families and 12 genera, while Spermatophyta has 634 taxa (96.6\%) belonging to 85 families and 337 genera. The distribution of these taxa to taxonomic units is shown in Table 2.

Table 2. The distribution of taxa into the large taxonomical groups

\begin{tabular}{lcccccccc}
\hline & Families & Genera & Species & Subsp. & Var. & Taxa* & Endemic & Rare \\
\hline Pteridophyta (a) & 10 & 12 & 22 & - & - & 22 & - & - \\
Spermatophyta (b) & 85 & 342 & 563 & 62 & 9 & 634 & 33 & 12 \\
Gymnosperms & 3 & 5 & 5 & - & 1 & 6 & - & - \\
Angiosperms & 82 & 337 & 558 & 62 & 8 & 628 & 33 & 12 \\
Magnoliids & 1 & 1 & 1 & - & - & 1 & - & - \\
Monocots & 14 & 61 & 93 & 11 & - & 104 & 3 & 3 \\
Eudicots & 67 & 275 & 464 & 51 & 8 & 523 & 30 & 9 \\
\hline Total (a+b) & 95 & 354 & 585 & 62 & 9 & 656 & 33 & 12 \\
*Including the lower taxa of the genera & & & & & & &
\end{tabular}

Of the 628 taxa of Angiospermae, one of which $(0.16 \%)$ belongs to Magnoliids, 104 (16.56\%) Monocots and 523 (83.28\%) Eudicots. Proportional distribution of the taxa belonging to these systematic groups are given below at the ordo level (Figures 4 and $5)$. 


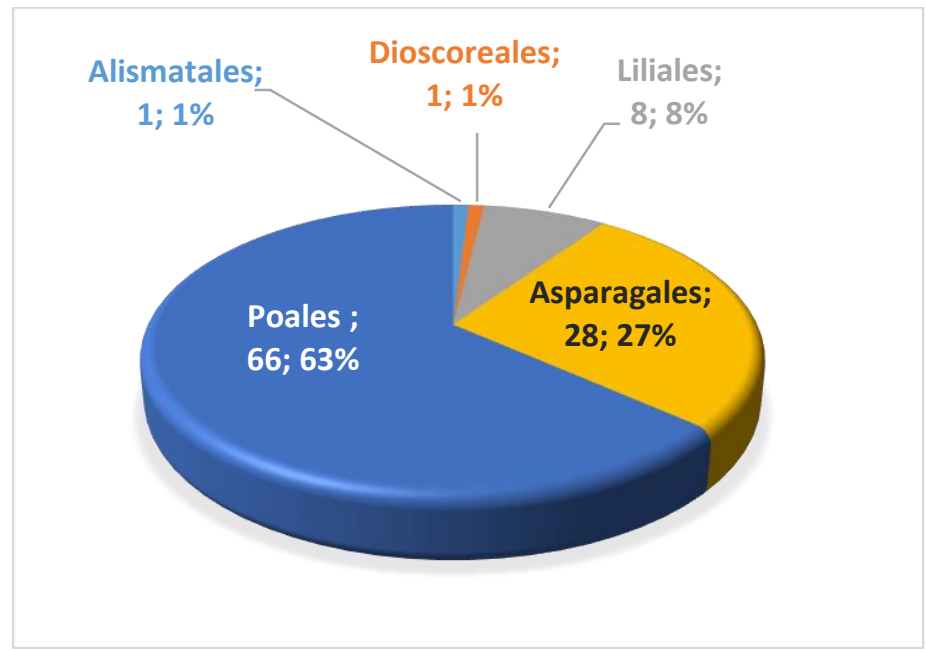

Figure 4. Proportional distribution of Monocots

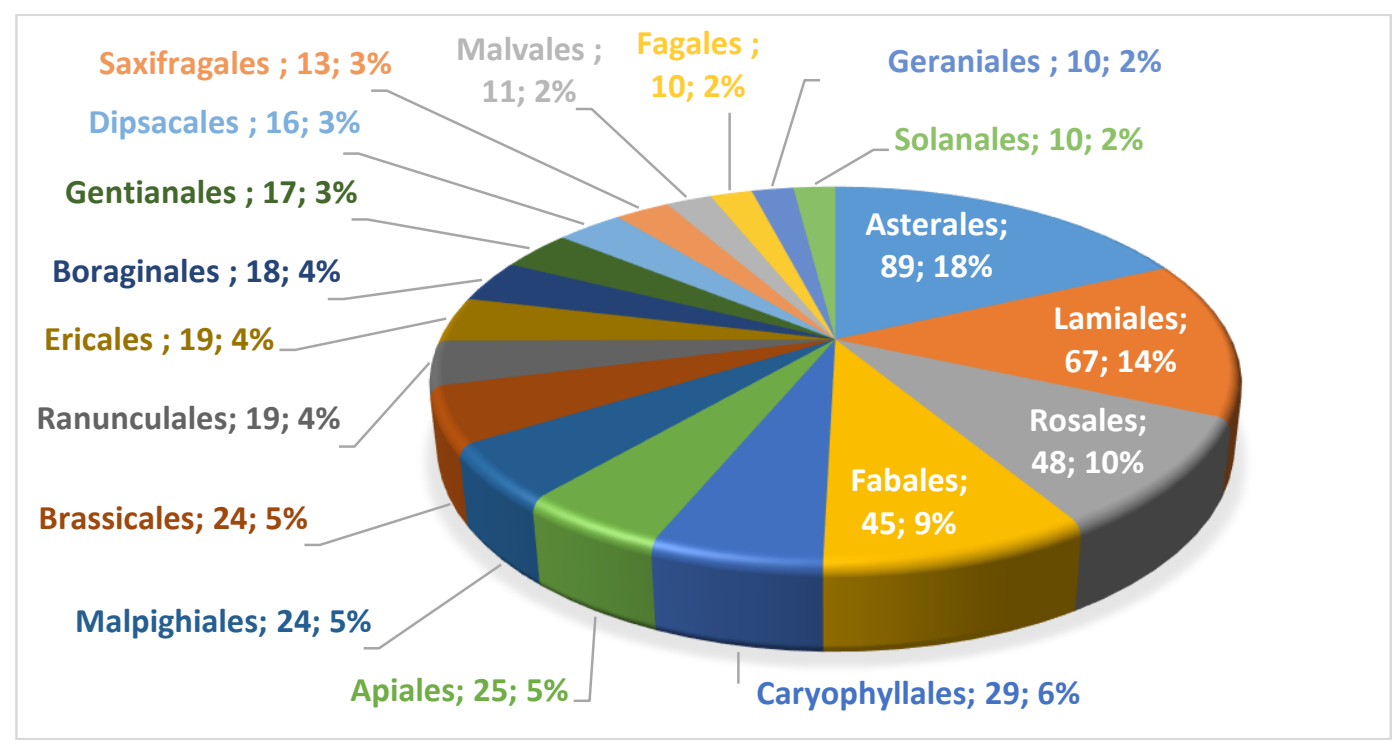

Figure 5. Proportional distribution of Eudicots

The most represented families and genera among the 95 families in terms of taxa richness are given in Table 3. Proportional comparison of leading families with the flora of Turkey is given in Figure 6.
Further analysis demonstrates the presence of 354 genera. Of these, Campanula (14), Carex (10), Alchemilla (9) and Geranium (9) have the greatest number of taxa (Table 3 ).

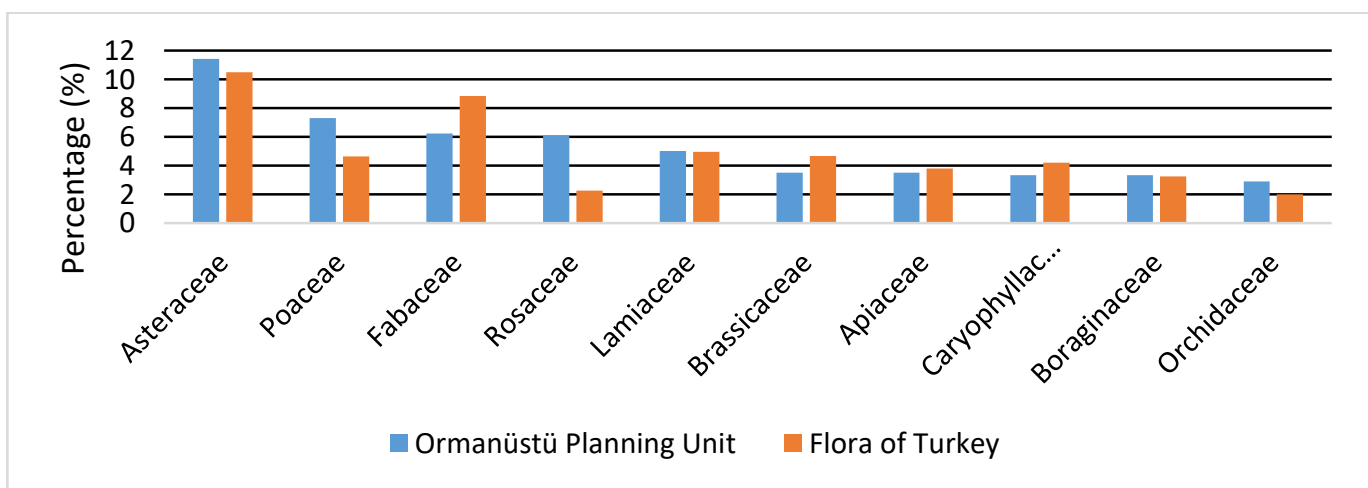

Figure 6. Comparison of plant families of the present study with flora of Turkey 
Table 3. Most represented families ( $>10$ taxa) and genera ( $\geq 5$ taxa) of the native vascular flora

\begin{tabular}{lclc}
\hline Families & Taxa & Genera & Taxa \\
\hline Asteraceae & 75 & Campanula L. & 14 \\
\hline Poaceae & 48 & Carex L. & 10 \\
\hline Fabaceae & 41 & Alchemilla L. & 9 \\
\hline Rosaceae & 40 & Geranium L. & 9 \\
\hline Lamiaceae & 33 & Veronica L. & 8 \\
\hline Brassicaceae & 23 & Trifolium L. & 7 \\
\hline Apiaceae & 23 & Euphorbia L. & 7 \\
\hline Caryophyllaceae & 22 & Myosotis L. & 7 \\
\hline Boraginaceae & 18 & Pilosella Hill. & 7 \\
\hline Orchidaceae & 15 & Medicago L. & 6 \\
\hline Campanulaceae & 14 & Clinopodium L. & 6 \\
\hline Ranunculaceae & 13 & Dryopteris Adans. & 5 \\
\hline Plantaginaceae & 13 & Poa L. & 5 \\
\hline Caprifoliaceae & 12 & Ranunculus L. & 5 \\
\hline Primulaceae & 11 & Sedum L. & 5 \\
\hline Orobanchaceae & 11 & Astragalus L. & 5 \\
\hline Total & 412 & Total & 115 \\
\hline
\end{tabular}

The phytogeographical regions of 336 taxa $(51.22 \%)$ were able to determine. Of these; 288 taxa (43.90\%) are Euro-Siberian element, 26 taxa $(3.96 \%)$ are Irano-Turanian element and 22 taxa $(3.36 \%)$ are Mediterranean element (Table 4). Mediterranean phytogeographical region is poorly represented in the study area.

Table 4. Numerical and proportional distributions of determined taxa according to the phytogeographical regions

\begin{tabular}{|c|c|c|c|c|c|c|}
\hline \multirow{2}{*}{ Phytogeographical regions } & \multirow{2}{*}{$\begin{array}{c}\text { Taxa } \\
\text { number }\end{array}$} & \multicolumn{2}{|c|}{ Ratio (\%) } & \multirow{2}{*}{$\begin{array}{c}\text { Taxa } \\
\text { number }\end{array}$} & \multicolumn{2}{|c|}{ Ratio (\%) } \\
\hline & & Flora* & $\mathrm{PhR}^{* *}$ & & Flora* & $\mathrm{PhR}^{* *}$ \\
\hline Euro Siberian & 153 & 23.32 & 45.54 & 288 & 43.90 & 85.71 \\
\hline Euro Siberian (mt.) element & 2 & 0.30 & 0.60 & & & \\
\hline Euxine element & 92 & 14.02 & 27.38 & & & \\
\hline Euxine (mt.) element & 22 & 3.35 & 6.55 & & & \\
\hline Hyrcano-Euxine element & 13 & 1.98 & 3.87 & & & \\
\hline Hyrcano-Euxine (mt.) element & 6 & 0.91 & 1.79 & & & \\
\hline Irano-Turanian & 26 & 3.96 & 7.73 & 26 & 3.96 & 7.74 \\
\hline Mediterranean & 21 & 3.20 & 6.25 & 22 & 3.36 & 6.55 \\
\hline Mediterranean (mt.) element & 1 & 0.16 & 0.30 & & & \\
\hline Sub-total (Determined) & 336 & 51.22 & 100 & - & - & \\
\hline Multi regional or unknown origin & 320 & 48.78 & & 320 & 48.78 & \\
\hline General Total & 656 & 100 & & 656 & 100 & 100 \\
\hline
\end{tabular}

Five alien species $(0.76 \%)$ were collected in the study area (Uludag et al., 2017). In addition, 17 cosmopolitan taxa (2.59\%) were determined in the study area.

\section{Endemic and Rare Plants}

Thirty-three endemic plants were found in the study area and the ratio of endemism is $5 \%$ (Table 5). In addition, 12 rare taxa (1.82\%) were identified and IUCN (2001) threat categories were indicated (Table 6). 
Table 5. IUCN threat categories and observed population densities of endemic taxa

\begin{tabular}{|c|c|c|c|}
\hline $\begin{array}{c}\text { IUCN } \\
\text { Categories }\end{array}$ & Taxa & Family & $\begin{array}{c}\text { Observed } \\
\text { Population Density }\end{array}$ \\
\hline CR & Astragalus ansinii & Fabaceae & SL \\
\hline EN & Alchemilla ikizdereensis & Rosaceae & SL \\
\hline EN & Astragalus ovatus & Fabaceae & DL \\
\hline VU & Hieracium gentiliforme & Asteraceae & SP \\
\hline NT & Symphytum sylvaticum subsp. sepulcrale & Boraginaceae & SW \\
\hline NT & Cirsium trachylepis & Asteraceae & DP \\
\hline NT & Lilium ciliatum & Liliaceae & SL \\
\hline NT & $\begin{array}{l}\text { Festuca amethystina subsp. orientalis var. } \\
\text { turcica }\end{array}$ & Poaceae & DP \\
\hline NT & Cyclamen parviflorum var. subalpinum & Primulaceae & DP \\
\hline NT & Melampyrum arvense var. elatius & Scrophulariaceae & DL \\
\hline NT & Sempervivum armenum var. armenum & Crassulaceae & SL \\
\hline NT & Centaurea helenioides & Asteraceae & SL \\
\hline $\mathrm{LC}$ & Veronica multifida & Scrophulariaceae & DL \\
\hline $\mathrm{LC}$ & Galium fissurense & Rubiaceae & DW \\
\hline $\mathrm{LC}$ & Epipactis turcica & Orchidaceae & SL \\
\hline $\mathrm{LC}$ & Cyclamen parviflorum var. parviflorum & Primulaceae & DP \\
\hline $\mathrm{LC}$ & Phlomis russeliana & Lamiaceae & SP \\
\hline $\mathrm{LC}$ & Onobrychis armena & Fabaceae & SL \\
\hline $\mathrm{LC}$ & Astragalus viridissimus & Fabaceae & SP \\
\hline $\mathrm{LC}$ & Geranium ibericum subsp. jubatum & Geraniaceae & SP \\
\hline $\mathrm{LC}$ & Geranium sintenisii & Geraniaceae & SP \\
\hline $\mathrm{LC}$ & Cerastium armeniacum & Caryophyllaceae & SL \\
\hline $\mathrm{LC}$ & Dianthus carmelitarum & Caryophyllaceae & DW \\
\hline $\mathrm{LC}$ & Arenaria kotschyana subsp. kotschyana & Caryophyllaceae & SL \\
\hline $\mathrm{LC}$ & Lonicera orientalis & Caprifoliaceae & DW \\
\hline $\mathrm{LC}$ & Campanula betulifolia & Campanulaceae & SL \\
\hline $\mathrm{LC}$ & Onosma bornmuelleri & Boraginaceae & SL \\
\hline $\mathrm{LC}$ & Hieracium karagoellense & Asteraceae & SL \\
\hline $\mathrm{LC}$ & Centaurea urvillei subsp. stepposa & Asteraceae & SL \\
\hline $\mathrm{LC}$ & Cirsium sommieri & Asteraceae & SP \\
\hline $\mathrm{LC}$ & Astrantia maxima subsp. haradjianii & Apiaceae & SL \\
\hline $\mathrm{LC}$ & Heracleum platytaenium & Apiaceae & DP \\
\hline $\mathrm{DD}$ & Hieracium subsilvularum & Asteraceae & SL \\
\hline
\end{tabular}

Table 6. Rare taxa and IUCN threat categories

\begin{tabular}{cllc}
\hline $\begin{array}{c}\text { IUCN } \\
\text { Categories }\end{array}$ & \multicolumn{1}{c}{ Taxa } & Family & $\begin{array}{c}\text { Observed } \\
\text { Population Density }\end{array}$ \\
\hline EN & Lilium monadelphum var. armenum & Liliaceae & SW \\
\hline VU & Ranunculus bulbosus subsp. aleae & Ranunculaceae & SP \\
\hline VU & Alchemilla speciosa & Rosaceae & DL \\
\hline VU & Alchemilla orthotricha & Rosaceae & DP \\
\hline VU & Alchemilla holotricha & Rosaceae & SP \\
\hline VU & Myosotis lazica & Boraginaceae & SP \\
\hline VU & Ruscus colchicus & Liliaceae & DP \\
\hline VU & Galanthus rizehensis & Liliaceae & DP \\
\hline VU & Osmanthus decorus & Oleaceae & SP \\
\hline VU & Anemone caucasica & Ranunculaceae & DP \\
\hline VU & Seseli petraeum & Apiaceae & SL \\
\hline DD & Laserpitium affine & Apiaceae & SP \\
\hline
\end{tabular}




\section{Taxa in BERN and CITES Lists}

With these international agreements, it is aimed to protect the species that are traded in the world or that may be under threat if protection measures are not taken. For this purpose, separate lists have been prepared for both treaties. The main goal is to protect the habitats with taxa in these lists and to prevent their international trade (Inskipp \& Gillett, 2005; WWF, 2009). Plant taxa determined in the study area according to the BERN (the shape formed with the supplements made in 1996 and 1997) and CITES lists are given in Table 7.

Table 7. Plant taxa in appendix of BERN convention and CITES list

\begin{tabular}{llccc}
\hline Family & \multicolumn{1}{c}{ Taxa } & $\begin{array}{c}\text { BERN } \\
\text { flora list }\end{array}$ & $\begin{array}{c}\text { CITES } \\
\text { flora list }\end{array}$ & $\begin{array}{c}\text { Observed } \\
\text { Population Density }\end{array}$ \\
\hline Ericaceae & Vaccinium arctostaphylos & + & - & DP \\
\hline Primulaceae & Cyclamen coum subsp. coum & + & + & DP \\
\hline Primulaceae & Cyclamen coum subsp. caucasicum & + & + & DP \\
\hline Primulaceae & Cyclamen parviflorum var. parviflorum & - & + & DP \\
\hline Primulaceae & Cyclamen parviflorum var. subalpinum & - & + & DP \\
\hline Liliaceae & Galanthus rizehensis & - & + & DP \\
\hline Orchidaceae & Ophrys oestrifera & + & - & SL \\
\hline Orchidaceae & Cephalanthera damasonium & - & + & SP \\
\hline Orchidaceae & Orchis simia & - & + & SL \\
\hline Orchidaceae & Orchis tridentata & - & + & DP \\
\hline Orchidaceae & Anacamptis pyramidalis & - & + & DP \\
\hline
\end{tabular}

(+) presence, (-) absence

\section{Discussion}

Invasions of alien species have negative impacts across several sectors such as agriculture, tourism, forestry, fishery, human health, water, and irrigation including artificial and natural habitats (Kariyawasam, 2019; Terzioğlu, 2020). These species have invasive tendency to naturalize and destructive effects on the natural food chain. Early growth is a predominant trait due to their early seed formation properties. They hamper the vitality of the natural species in the region and decrease biodiversity (Terzioğlu \& Anşin, 2001). They can change the entire habitat and put the ecosystem at risk. They are also considered as the driving force of recent extinctions (Bellard et al., 2016).

The ratio of alien plants in the study area is very low $(0.76 \%)$, that is compatible with the ratio $(0.8 \%)$ of the nearby study (Uzun \& Terzioğlu, 2008). The study area mostly consists of closed shady forest areas, offering relatively less suitable areas for alien plants, also has unsuitable habitat conditions at high elevations, and low light intensity due to the closed forest cover causes native plants to limit the entry of other alien plants into the forests (Mavimbela et al., 2018). Compared with the total alien flora of Turkey $(2.9 \% ; 340$ alien plants against 11,707 total flora) there was also no significant difference (Güner et al., 2012; Uludağ et al., 2017).

Robinia pseudoacacia and Ailanthus altissima are the most worrisome woody taxa found in the study area. Since they are tree species, they can remain in the area where they are established for a long time and act as a bank of seed (Gioria et al., 2012). Less worrying taxa in the area are Erigeron canadensis and Erigeron annuus. These taxa are less important because they are annual and high light demanding plants and they cannot get into the forest ecosystems easily due to low light availability. The last of the alien species identified in the study area is Opuntia ficus-indica. It was observed in the area where this taxon was first brought for garden design but then spread to surrounding areas. This taxon is spread by its tubers. For this reason, it is considered that it may pose a threat to the native plant species in the region. Compared to other alien plants in Turkey, the abovementioned tree species seem to be the most common species in forest ecosystems in the Black Sea Region (Terzioğlu \& Anşin, 2001). This is because Robinia pseudoacacia has 
been planted on forest roadsides for many years as part of forestry (Kalay, 1987).

Cosmopolitan taxa show wide ecological tolerance and can produce new individuals that can even spread long distances. Of those worth to mention; Tanacetum parthenium, Cichorium intybus, Capsella bursa-pastoris, Convolvulus arvensis, Verbena officinalis, Datura stramonium, Solanum nigrum, located at road sides and openings, while Trifolium pratense, Vicia sativa, Cerastium fontanum, Silene gallica, at meadow areas, Veronica anagallis-aquatica, Polygonum aviculare, Juncus effusus, Juncus bufonius, spread in humid-wet habitats. Galium aparine is found as a climber on other species.
Güner et al. (2012) states that some taxa which we included in our study are no longer endemic to the country, based on recent research and new taxonomic information from neighbouring countries. However, we found it appropriate to include these taxa in the endemic list due to their narrow global population. These taxa are as follows; Veronica multifida, Centaurea urvillei subsp. stepposa, Heracleum platytaenium, Astragalus ovatus, Lamium ponticum. Actually, it would be more meaningful to evaluate these taxa, which are omitted from the endemic list of Turkey, in the rare category if they are not synonymous.

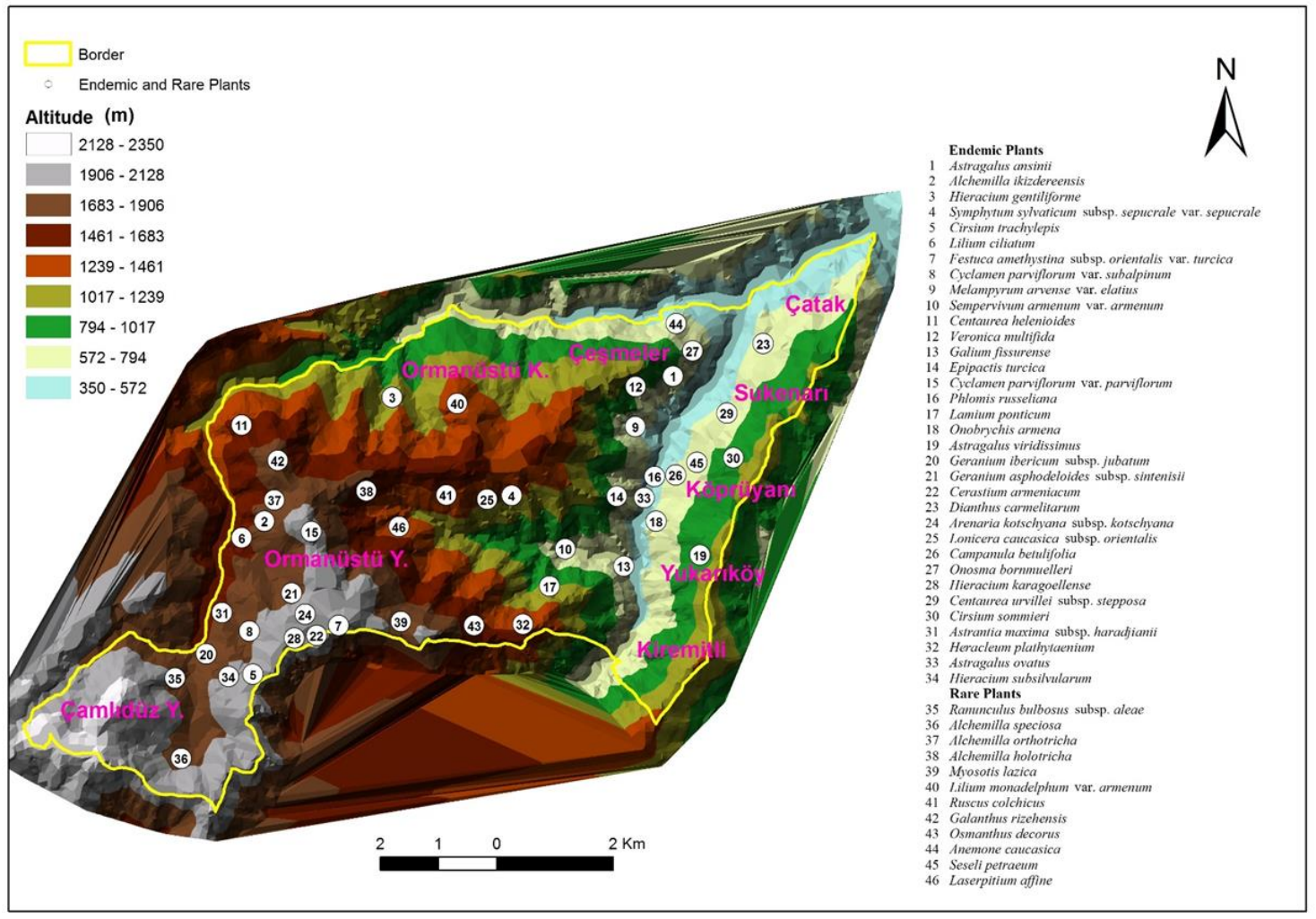

Figure 7. Localities of endemic and rare taxa

Plant endemism in the present study was found at a very low ratio (5\%). As the main reasons, high rainfall and dense closed forests limit the endemism in the Black Sea Region, especially in the eastern side "Colchis as a term" (Anşin, 1983). Floristic studies conducted in the Eastern Black Sea Region reveal that the endemism ratio in the coastal areas of the Eastern Black Sea Mountains varies between roughly $3-9 \%$. E.g; It is $3.2 \%$ in Palabaş-Uzun \& Terzioğlu (2019), 4.2\% in Uzun \& Terzioğlu (2008), 5.3\% in Demir \& Atamov (2019) and 8.3\% in Palabaş-Uzun \& Anşin (2006). In the terrestrial parts of the mountains, the endemism ratio exceeds $10 \%$. E.g; $10.03 \%$ in Karaer \& K1lınç (2001). It is estimated that there are approximately 500 endemic taxa in the Black Sea region (Anşin, 1982). Türe \& Böcük (2010) also reported that 
the ratio of endemism is 5\% in the entire EuroSiberian area of Turkey.

The majority of endemic plants in the present study are in the Least Concern (LC) threat category (IUCN, 2001). Besides, eight (8) taxa were determined in the Near Threatened (NT) category. Populations of Lilium ciliatum and Centaurea helenioides were determined from the only one locality considering the distribution of these taxa in the study area. The threat category of Lilium ciliatum, which is in NT according to Ekim et al. (2000), was later suggested as EN in a revision study (İkinci, 2005). Our field observations support the last amendment. Melampyrum arvense var. elatius, Cyclamen parviflorum var. subalpinum and Cirsium trachylepis are the densest taxa in the study area for NT category.

Hieracium gentiliforme is in the Vulnerable (VU) threat category and its narrow populations are fragmented in forest patches. It was found in three sub-localities dominated by Oriental spruce (Picea orientalis) between the elevations of 1614$1940 \mathrm{~m}$. Alchemilla ikizdereensis is in the Endangered (EN) threat category and populations of this species are at risk of extinction in the near future. The newly discovered Astragalus ansinii is in the Critically Endangered (CR) threat category (CR: B2b (ii, iv)), which at risk of extinction in a very close future. It is still known from two close subpopulations in the degraded pseudomacchie vegetation of the study area, with Medit.-Euxine shrubs and short trees (Uzun et al., 2009). Habitat loss and fragmentation are two biggest threat that will cause this taxon to disappear in near future. Tourism intensity is another threat to many species because the area has high religious / cultural sites such as the Vazelon monastery.

Two taxa, which are in the Data Deficient (DD) category according to Ekim et al. (2000), were re-collected in this study and new information was obtained (IUCN 2001). The first one; Astragalus ovatus was recollected more than 100 years later after Sintenis, in a low-elevation stony-meadow areas in only of pseudomacchie vegetation in the study area. Its population is dense but local throughout the study area. The threat category of this species was (DD) firstly, then changed to (EN) by Ekici (2010). We also approve this amendment. The second one was Hieracium subsilvularum. This taxon was determined at $1710 \mathrm{~m}$ in mixed old forest stands consisting of Oriental spruce (Picea orientalis (L.) Link.) and Oriental beech (Fagus orientalis Lipsky) trees. VU category is appropriate for this taxon, which is thought to be affected by intensive forestry activities.

Most of the rare taxa identified in this study are in the Vulnerable (VU) threat category. Rare plants, which is also known from neighbouring countries, are the taxa whose populations are thought to be threatened in the future (Table 6). However, evaluations in Red Data Book of Turkish Plant (Ekim et al., 2000) was mostly made according to the distribution and population status in Turkey. Therefore, it is necessary to re-evaluate the existing threat categories according to IUCN (2001) criteria both on a spatial and numerical scale. In this regard, İkinci (2005) had previously proposed a change of the threat category of Lilium monadelphum var. armenum from VU to EN. However, this taxon now is accepted as Lilium armenum, which distribute in the Transcaucasian countries (Azerbaijan + Armenia + Georgia) and in Turkey.

Eleven taxa that are included in the annexes of BERN and CITES conventions have been identified in the study area. Four of them (Vaccinium arctostaphylos, Cyclamen coum subsp. coum, Cyclamen coum subsp. caucasicum, Ophrys oestrifera) are in the appendix of BERN convention and the protection activities regarding the critical habitats of these species are guaranteed by this agreement. Nine taxa from the study area are listed in the CITES annexes (Cyclamen coum subsp. coum, Cyclamen coum subsp. caucasicum, Cyclamen parviflorum var. parviflorum, Cyclamen parviflorum var. subalpinum, Galanthus rizehensis, Cephalanthera damasonium, Orchis simia, Orchis tridentata, Anacamptis pyramidalis). For these taxa, illegal uprooting and collection activities, if any, should be prevented. Forest engineer carrying out conservation activities in the field should be informed in this regard. For this, primarily the materials such as brochures and posters should be provided to the interest groups to recognize these taxa and 
necessary warnings should be made especially for local and touristic people for the protection of these species.

\section{Conclusions}

This study contributes to understanding the floristic structure of the Colchis sector (east of the Euxine phytogeographical region) and it has been concluded that the study area is also important in terms of plant diversity within the Colchic region. The discovery of a new species (Astragalus ansinii Uzun, Terzioğlu $\&$ Pal.-Uzun) from the study area supports this fact. This also shows that detailed flora studies are still needed for the protection of world plant biodiversity.

Determining the plant richness and diversity in an area is primarily necessary for transferring the assets of natural plant taxa to future generations, protecting and surviving target plants that require conservation measures, functional forest planning and management, silvicultural practices and even for beekeeping efforts.

Degraded broad-leaved forest stands in rocky and stony places provided a suitable growing condition for many endemic species in the study area. For this reason, such areas should be considered as plant refugia and destructive activities such as clear-cutting, vegetation cleaning and afforestation using foreign-origin trees should be avoided.

In all kinds of technical activities in the study area, target plant species such as Astragalus ansinii, A. ovatus, Lilium ciliatum, Cyclamen parviflorum, Hieracium gentiliforme, Hieracium subsilvularum, Osmanthus decorus (relict) and Epipactis turcica should be protected.

\section{Acknowledgement}

This study is a part of the first author's $\mathrm{PhD}$ dissertation. In this process, valuable help was received from Dr. Zeki Aytaç and Dr. Murat Ekici for the expertise of the genus Astragalus, from Dr. Kamil Coşkunçelebi for the genus Hieracium and from Dr. Fergan Karaer for the genus Sempervivum in Turkey.

\section{Ethics Committee Approval N/A}

\section{Peer-review \\ Externally peer-reviewed.}

\section{Author Contributions}

Conceptualization: A.U.; Investigation: A.U.; Material and Methodology: A.U.; Supervision: S.T.; Visualization: A.U., S.T.; Writing-Original Draft: A.U.; Writing-review \& Editing: A.U., S.T.; Other: All authors have read and agreed to the published version of manuscript.

\section{Conflict of Interest}

The authors have no conflicts of interest to declare.

\section{Funding}

The authors declared that this study has received financial support from Karadeniz Technical University Scientific Research Commission (KTU-BAP-2003-113-001-6).

\section{References}

Akçiçek, E. (2020). Taxonomic revision of Stachys sect. Olisia (Lamiaceae: Lamioideae) in Turkey. Phytotaxa, 449, 2, 109-148.

Akman, Y. (1990). Iklim ve Biyoiklim, Palme Yayınları, Mühendislik Serisi: 103, Ankara, 319s.

Al-Shehbaz, I.A., Mutlu, B. \& Dönmez, A.A. (2007). The Brassicaceae (Cruciferae) of Turkey, updated. Turkish Journal of Botany, 31, 327-336.

Altun, L., Başkent, E.Z., Yılmaz, M., Kalay, H.Z. \& Turna, İ. (2002). KTÜ Ormanüstü araştırma ormanında yetişme ortamı birimlerinin coğrafi bilgi sistemleri yardımıyla haritalanması. Ü.O.F. Dergisi, A, 52, 2, 51-72.

Anşin, R. (1980). Doğu Karadeniz Bölgesi florası ve asal vejetasyon tiplerinin floristik içerikleri. Thesis, Karadeniz Technical University, Trabzon, Turkey.

Anșin, R. (1982). Endemizm ve Doğu Karadeniz Bölgesinde yetișen endemik bitki taksonları, (Endemism and endemic plants of the East Black Sea region), KTÜ Orman Fakültesi Dergisi, 5, 2, 311-326.

Anşin, R. (1983). Türkiye'nin flora bölgeleri ve bu bölgelerde yayılan asal vejetasyon tipleri (The Floristic regions and the major vegetation types of Turkey). KTÜ Orman Fakültesi Dergisi, 6, 2, 318-339.

Bagnouls, F. \& Gaussen, H. (1957). Les climats biologiques et leur classification. Ann Georgr 66, 193-220. 
Banasiak, L., Wojewódzka, A., Baczyński, J., Reduron, J.-P., Piwczyński, M., KurzynaMłynik, R., Gutaker, R., Czarnocka-Cieciura, A., Kosmala-Grzechnik, S. \& Spalik, K. (2016). Phylogeny of Apiaceae subtribe Daucinae and the taxonomic delineation of its genera. Taxon, 65, 3, 563-585.

Bartolucci, F., Peruzzi, L., Galasso, G., Albano, A., Alessandrini, A., Ardenghi, N.M.G., Astuti, G., Bacchetta, G., Ballelli, S., Banfi, E. \& Conti, F. (2018). An updated checklist of the vascular flora native to Italy. Plant Biosystems, 152, 2, 79-303.

Baytop, A. (1998). Ingilizce-Türkçe botanik kllavuzu. İ̈̈ Basımevi ve Film Merkezi, Üniversite Yayın No: 4058, Eczacılık Fak. Yayın No: 70, İstanbul, $375 \mathrm{~s}$.

Baytop, T. (1994). Türkçe bitki adları sözlüğü. Türk Tarih Kurumu Basımevi, Türk Dil Kurumu Yayınları: 578, Ankara, 508 s.

Bellard, C., Cassey, P. \& Blackburn, T.M. (2016). Alien species as a driver of recent extinctions. Biology Letters 12, 2, 20150623.

BERN Convention (1979). https://www.coe.int/en/web/conventions/fulllist/-/conventions/treaty/104 (accessed 15.09.2020)

Boissier, E. (1867-88). Flora Orientalis, 1-5. Suplement by Buser, R. Geneve, Switzerland.

Bonnier, G. (1912-1934). Flore complete illustree en couleurs de France Suisse et Belgique, IXII. Neuchatel, Paris, Bruxelles.

Bräutigam, S. \& Greuter, W. (2007). A new treatment of Pilosella for the EuroMediterranean flora. Willdenowia, 37, 123137.

Chase, M.W., Christenhusz, M., Fay, M., Byng, J., Judd, W.S., Soltis, D., Soltis, P.S. \& Stevens, P.F. (2016). An update of the angiosperm phylogeny group classification for the orders and families of flowering plants: APG IV. Botanical Journal of the Linnean Society, 181, 1, 1-20.

Coşkunçelebi, K. (2001). Doğu Karadeniz bölgesinde yayılış gösteren Hieracium L. (Compositae) türlerinin morfolojik ve nümerik taksonomik yönden incelenmesi, $\mathrm{PhD}$ thesis, Karadeniz Technical University, Trabzon, Turkey.

Coşkunçelebi, K. (2003). New combinations in the genus Hieracium L. stricto and Pilosella Hill (Asteraceae) in Turkey. Annales Botanici Fennici, 40, 451-453.

Coşkunçelebi, K. \& Beyazoğlu, O. (2001). New Hieracium L. (Asteraceae) records for the flora of Turkey. Turkish Journal of Botany, 25, 249253.
Coşkunçelebi, K. \& Beyazoğlu, O. (2003). Assessment of phenetic clustering in Hieracium L. s.str. (Asteraceae) species distributed in Black Sea region of Turkey. Bulletin of the Polish Academy of Sciences, 51, 17-24.

Çavuş, A. (2016). A Lesser known important cultural heritage source and religious tourism value in Turkey: Vazelon (Zavulon) Monastery. International Journal of Humanities and Social Science, 6, 1, 190-199.

Davis, P.H. (1965-85). Flora of Turkey and the East Aegean Islands, I-IX. Edinburgh University Press, Edinburgh.

Davis, P.H., Mill, R.R. \& Tan, K. (1988). Flora of Turkey and the East Aegean Islands, X, supplement. Edinburgh University Press, Edinburgh.

Demir, E. \& Atamov, V. (2019). AyderCeymakçur yaylaları (Çamlıhemşin / Rize) arasında kalan bölgenin florası. Journal of Anatolian Environmental and Animal Sciences, 4, 2, 201-210.

Eken, G., Bozdoğan, M., İsfendiyaroğlu, S., Kılıç, D.T. \& Lise, Y. (2006). Türkiye'nin önemli doğa alanlart. Doğa Derneği, Ankara.

Ekici, M. (2010). Astragalus L. (Fabaceae) cinsinin Hypoglottidei DC., Incani DC. ve Dissitiflori DC. seksiyonlarında yer alan tükenmiş (EX) ve yetersiz veri (DD) basamağında bulunan taksonlar ile ilgili veriler. SAÜ Fen Edebiyat Dergisi, 1, 31-42.

Ekim, T., Koyuncu, M., Vural, M., Duman, H., Aytaç, Z. \& Adigüzel, N. (2000). Türkiye bitkileri kırmızı kitabl (Red data book of Turkish plants Pteridophyta and Spermatophyta). Barışcan Ofset, Ankara, 246 s.

Erata, H., Batan, N., Alataş, M. \& Özen, Ö. (2020). Bryophytes records from Maçka district (Trabzon province-Turkey) Lindbergia 43, doi: 10.25227/ linbg.01127.

Erinç, S. (1969). Klimatoloji ve metodları. İÜ Coğ. Enst. Yay., No: 35, İstanbul.

Euro+Med. (2006 onwards). Euro+Med Plantbase - the information resource for EuroMediterranean plant diversity. http://ww2.bgbm.org/EuroPlusMed/ (accessed 10.09.2020).

Farjon, A. (1998; 2nd ed. 2001). World checklist and bibliography of conifers. Royal Botanic Gardens, Kew

Farjon, A. (2018). Conifers of the world. Kew Bulletin, 73, 8 .

Fitter, R., Fitter, A. \& Blamey, M. (2000). Parey blumenbuch, Blütenpflanzen Deutschlands und Nordwesteuropas, 3. Auflage. Parey Buchverlag, Berlin. 
Frajman, B., Thollesson, M. \& Oxelman, B. (2013). Taxonomic revision of Atocion and Viscaria (Sileneae, Caryophyllaceae). Botanical Journal of the Linnean Society, 173, 194-210.

Funk, V.A. (2006). Floras: A model for biodiversity studies or a thing of the past? Taxon, 55, 581-588.

GDM, (2021). General Directorate of Meteorology of Turkey. https://www. mgm.gov.tr (accessed 26.02.2021)

Gioria, M., Pyšek, P. \& Moravcová, L. (2012). Soil seed banks in plant invasions: promoting species invasiveness and long-term impact on plant community dynamics. Preslia 84, 327350.

Gutiérrez-Larruscain, G., Santos-Vicente, M., Martínez-Ortega, M.M. \& Rico, E. (2019). Typification of 25 names in Inula (Inuleae, Asteraceae), and a new combination in Pentanema. Phytotaxa, 395, 1, 17-26.

Güner, E.D. (2006). Türkiye'deki Seseli L. (Umbelliferae) cinsinin revizyonu, $\mathrm{PhD}$ thesis, Gazi University, Ankara, Turkey.

Güner, A., Aslan, S., Ekim, T., Vural, M., Babaç, M.T. (2012). Türkiye bitkileri listesi (Damarlı bitkiler). Flora Araştırmaları Derneği ve Nezahat Gökyiğit Botanik Bahçesi Yayını, İstanbul, Turkey. (in Turkish)

Güner, A., Özhatay, N., Ekim, T. \& Başer, K.H.C. (2000). Flora of Turkey and the East Aegaen Islands, XI, supplement-II. Edinburgh University Press, Edinburgh.

Güven, İ.H. (1993). Doğu pontidlerin jeolojisi ve 1/250,000 ölçekli kompilasyonu. MTA yayınları, Ankara.

Hayırlığlu-Ayaz, S. (1997). Doğu Karadeniz bölgesinde yayulış gösteren Alchemilla türlerinin morfolojik ve sitotaksonomik yönden incelenmesi, $\mathrm{PhD}$ thesis, Karadeniz Technical University, Trabzon, Turkey.

Hayırlığlu-Ayaz, S. \& Beyazoğlu, O. (2002). Two new Alchemilla L. (Rosaceae) records for the flora of Turkey. Turkish Journal of Botany, 26, 47-50.

Hayırlığlu-Ayaz, S. \& Kalheber, H. (2002). Six new Alchemilla species from northeast Anatolia. Sendtnera, 8, 59-75.

Hegi, G., Merxmüller, H. \& Reisigl, H. (1977). Alpenflora.Verlag Paul Parey, Berlin und Hamburg.

Hernandez-Ledesma, P., Berendsohn, W.G., Borsch, T., von Mering, S., Akhani, H., Arias, S., Castaneda-Noa, I., Eggli, U., Eriksson, R., Flores-Olvera, H., et al. (2015). A taxonomic backbone for the global synthesis of species diversity in the angiosperm order Caryophyllales. Willdenowia, 45, 281-383.
İkinci, N. (2005). Revision of the genus Lilium L. (Liliaceae) in Turkey. PhD thesis, Abant İzzet Baysal University, Department of Biology, Bolu, Turkey.

Inskipp, T. \& Gillett, H.J. (eds.) (2005). Checklist of CITES species and annotated CITES appendices and reservations, Compiled by UNEP-WCMC, CITES Secretariat, Geneva, Switzerland and UNEP-WCMC, Cambridge, UK, 339.

IPNI, (2012 onwards) The International plant names index. http://www.ipni.org. (accessed 15.09.2020)

Irmak, A. (1974). Trabzon orman başmüdürlüğü çevresinde 27.9.1971-2.10.1971 tarihleri arasında yapılan tatbikatlara ait bazı ekolojik gözlemler. IÜ Orman Fakültesi Dergisi, B, 24, 2, 1-16.

IUCN Species Survival Commission, (2001). IUCN Red list categories, Ver. 3.1., IUCN, 118.

Kalay, Z. (1987). Ülkemiz ekolojik şartlarına uyumu ve ilginç özellikleri bakımından önemli bir yabanc1 ağaç türünden faydalanma imkanları. Orman Mühendisliği Dergisi, 8, 3541.

Karaer, F. \& Celep, F. (2008). Sempervivum ekimi nom. et stat. nov. for $S$. minus var. glabrum (Crassulaceae), with an amplified description. Annales Botanica Fennici, 45, 229-232.

Karaer, F. \& Kilınç, M. (2001). The Flora of Kelkit valley. Turkish Journal of Botany, 25, 195-238.

Kariyawasam, C.S., Kumar, L. \& Ratnayake, S.S. (2019). Invasive plants distribution modeling: A tool for tropical biodiversity conservation with special reference to Sri Lanka. Tropical Conservation Science, 12, 1-12.

Kaya, A. (2003). The Genus Astrantia L. in Turkey: morphology and anatomy. Acta Botanica Croatia, 62, 2, 89-102.

Komarov, V.L. (1934-1978). Flora of the U.S.S.R., 1-30. Israel Program for Scientific Translations, Jerusalem.

Kučera, J., Lihová, J. \& Marhold, K. (2006). Taxonomy and phylogeography of Cardamine impatiens and $C$. pectinata (Brassicaceae). Botanical Journal of Linnean Society, 152, 169-195.

Lambeck, R.J. (1997). Focal species: A multispecies umbrella for nature conservation. Conservation Biology, 11, 4, 849-856.

Luebert, F., Cecchi, L., Frohlich, M.W., Gottschling, M., Guilliams, C.M., HasenstabLehman, K.E., Hilger, H.H., Miller, J.S., Mittelbach, M., Nazaire, M., et al. (2016). Familial classification of the Boraginales. Taxon, 65, 502-522. 
Mavimbela, L.Z., Sieben, E.J.J. \& Proches, S. (2018). Invasive alien plant species, fragmentation and scale effects on urban forest community composition in Durban, South Africa. New Zealand Journal of Forestry Science, 48, 19.

McNeill, J., Barrie, F.R., Buck, W.R., Demoulin, V., Greuter, W., Hawksworth, D.L., Herendeen, P.S., Knapp, S., Marhold, K., Prado, J., et al., editors. (2012). International code of nomenclature for algae, fungi and plants (Melbourne code) adopted by the eighteen international botanical congress Melbourne, Australia, July 2011. Konigstein: Koeltz Scientific Books. (Regnum Vegetabile; 154).

Moore, A.J. \& Dillenberger, M.S. (2017). A conspectus of the genus Cherleria (Minuartia s.1., Caryophyllaceae). Willdenowia, 47, 1, 514.

Myers, N., Mittermeier, R.A., Mittermeier, C.G., da Fonseca, G.A.B. \& Kent, J. (2000). Biodiversity hotspots for conservation priorities. Nature, 403, 853-858.

OGM, (2006). KTÜ Araştırma ormanı işletme şefliği amenajman plant.

Olson, D.M. \& Dinerstein, E. (2002). The Global 200: Priority ecoregions for global conservation. Annals of the Missouri Botanical Garden, 89, 199-224.

Olson, D.M., Dinerstein, E., Wikramanayake, E.D., Burgess, N.D., Powell, G.N., Underwood, E.C., D'amico, J.A., Itoua, I., Strand, H.E., Morrison, J.C., Loucks, C.J., Allnutt, T.F., Ricketts, T.H., Kura, Y., Lamoreux, J.F., Wettngel, W.W., Hedao, P. \& Kassem, K.R. (2001). Terrestrial ecoregions of the world: A new map of life on earth, BioScience, 51, 11.

Özer, Z., Tursun, N., Önen, H., Uygur, F.N. \& Erol, D. (1998). Herbaryum yapma teknikleri ve yabancl ot teşhis yöntemleri. Gaziosmanpaşa Üniversitesi, Ziraat Fakültesi Yayınları No: 22, Kitaplar Serisi No: 12, Tokat, 214s.

Öztan, Y. (1980). Meryemana Deresi havzasindaki mera ve orman arazisinde otlatmanın değişik etmenlerle ilişsili olarak fiziksel ve hidrolojik toprak özellikleri üzerindeki etkileri. KTÜ Orman Fakültesi Matbaas1, Trabzon.

Palabaş-Uzun, S. \& Anşin, R. (2006). Subalpine and alpine flora of Altındere valley (Maçka, Trabzon). Turkish Journal of Botany, 32, 135158.

Palabaş-Uzun, S. \& Terzioğlu, S. (2019). Flora of Sisdağ1 (Salpazar1/Trabzon) and environs.
Düzce Üniversitesi Bilim ve Teknoloji Dergisi, 7, 1523-1573.

Palabaş-Uzun, S., Uzun, A. \& Terzioğlu, S. (2012). Orman ekosistemlerinde habitat parçalanmaları ve biyolojik çeşitlilik üzerine etkileri. KSÜ Doğa Bil. Der., Özel Sayı, 136144.

Pelin, S. (1977). Alucra (Giresun) güneydoğu yöresinin petrol olanaklarl bakımından incelenmesi, Doçentlik tezi, KTÜ Yer Bil. Fak. No: 13, Trabzon.

Phillips, R. (1994). Grasses, ferns, mosses \& lichens of Great Britain and Ireland, second edition. Macmillan Publishers Ltd., London.

PPG I (2016). A community-derived classification for extant lycophytes and ferns. Journal of Systematics and Evolution, 54, 563-603.

Rechinger, K.H. (1965-1977). Flora Iranica. Akadedemische Druck- u Verlagsanstalt.

Reveal, J.L. (2011). Summary of recent systems of angiosperm classification. Kew Bulletin, 66, 548.

Roma-Marzio, F., Najar, B., Alessandri, J., Pistelli, L. \& Peruzzi, L. (2017). Taxonomy of prickly juniper (Juniperus oxycedrus group): A phytochemical-morphometric combined approach at the contact zone of two cryptospecies. Phytochemistry, 141, 48-60.

Speta, F. (1998). Systematische analyse der Gattung Scilla-L. s.l. (Hyacinthaceae). Phyton, Annales Rei Botanicae, Horn, 38, 1, 1-141.

Terzioğlu, S. (2020). Arrhenatherum elatius subsp. bulbosum (Poaceae): A new alien record for the flora of Turkey. KSU Journal of Agriculture and Nature, 23, 4, 924-927.

Terzioğlu, S. \& Anşin, R. (2001). A chorological study on the taxa naturalized in the Eastern Black Sea region. Turkish Journal of Botany, 25, 305-309.

The Plant List. (2013 onwards). The plant list.,version 1.1. http://www.theplantlist. org/ (accessed 06.09.2020).

Thiers, B. (2020). Index herbariorum: A Global directory of public herbaria and associated staff. Available from: http:// sweetgum.nybg.org/ih/ (accessed 06.09.2020).

Thulin, M., Jarvis, C.E., Jonsell, B. \& Ryman, S. (2009). The status of "Frangula dodonei" (Rhamnaceae). Taxon, 58, 3, 991-992.

Topraksu Genel Müdürlüğü, (1981). Doğu Karadeniz havzası toprakları. Topraksu Genel Müd. Yayın No: 310, Ankara.

Tutin, T.G., Heywood, V.H. \& Burgers, N.A. (1964-1980). Flora Europaea, 1-5. Cambridge University Press.

Tutin, T.G., Burges, N.A., Chater, A.O., Edmondson, J.R., Heywood, V.H., Moore, D.M., Valentine, D.H., Walters, S.M., Webb, 
D.A., Akeroyd, J.R., Newton, M.E. \& Mill, R.R. (1993). Flora Europaea, 1, second edition. Cambridge: Cambridge University Press.

Türe, C., Böcük, H. (2010). Distribution patterns of threatened endemic plants in Turkey: A quantitative approach for conservation. Journal for Nature Conservation, 18, 296-303.

Uzun, A. \& Terzioğlu, S. (2008). Vascular flora of forest vegetation in Altındere Valley (MaçkaTrabzon). Turkish Journal of Botany, 32, 135158.

Uzun, A., Terzioğlu, S., Palabaş-Uzun, S. \& Coşkunçelebi, K. (2009). Astragalus ansinii sp. nov. (Fabaceae) from Turkey, and a contribution to the sectional taxonomy, Nordic Journal of Botany, 27, 5, 397-401.

Uludağ, A., Aksoy, N., Yazlık, A., Arslan, Z.F., Yazmış, E., Üremiş, I., Cossu, T.A., Groom, Q., Pergl, J., Pyšek, P. \& Brundu, G. (2017). Alien flora of Turkey: checklist, taxonomic composition and ecological attributes. NeoBiota, 35, 61-85.

Wang, R. (2014). A new combination in Alkekengi (Solanaceae) for the flora of China. Phytotaxa, 178, 1, 59-60.
WCSP (2020). World checklist of selected plant families. Facilitated by the Royal Botanic Gardens, Kew. Published on the internet;http://wcsp.science.kew.org/ (accessed 15.09.2020)

Wei, R., Ebihara, A., Zhu, Y.-M., Zhao C.-F., Hennequin, S. \& Zhang, X.-C. (2017). A totalevidence phylogeny of the lady fern genus Athyrium Roth (Athyriaceae) with a new infrageneric classification. Molecular phylogenetics and evolution, doi: 10.1016/j.ympev.2017.10.019

WWF, (2009). Kafkasya-ekolojik-bölgesi https://www.wwf.org.tr/ne_yapiyoruz/doga_k oruma/ (accessed 25.07.2020)

Yaltırık, F. \& Efe, A. (1996). Otsu bitkiler sistematiği ders kitabl, İ.Ü. Fen Bilimleri Enstitüsü Yayınları, İ.Ü. Yayın no: 3940, Orman fakültesi yayın no: 10, İstanbul.

Yolasığmaz, H.A. (1998). Coğrafi bilgi sistemi ile orman fonksiyon haritalarının hazırlanması, MSc thesis, Karadeniz Technical University, Trabzon, Turkey.

Zidorn, C. (2012). Leontodon and Scorzoneroides (Asteraceae, Cichorieae) in Italy. Plant Biosystems, 146, Suppl., 41-51. 


\section{APPENDIX (FLORISTIC LIST)}

\section{FERN AND FERN ALLIES}

Equisetales DC. ex Bercht. \& J.Presl F1 [1]. Equisetaceae

\section{Equisetum L. - At kuyruğu}

1. Equisetum arvense L.

Sukenar1 district, damp creek side, $640 \mathrm{~m}$, 07.07.2008, Uzun 1116 (KATO: 17481).

2. Equisetum telmateia Ehrh.

Köprüyanı district, forest edge damp place, 1150 m, 11.07.2007, Uzun 967 (KATO: 17482).

Ophioglossales Link

\section{F2 [2]. Ophioglossaceae}

\section{Botrychium $\mathrm{Sw}$}

3. Botrychium lunaria (L.) Sw.

Ormanüstü plateau, grassy place, $1880 \mathrm{~m}$, 10.06.2005, Uzun 618 (KATO: 17483).

Polypodiales Link

\section{F3 [3]. Pteridaceae}

\section{Adiantum L.}

4. Adiantum capillus-veneris $\mathrm{L}$. Çatak, rock cracks, 400 m, 11.07.2008, Uzun 1402 (KATO: 17484).

\section{F4 [4]. Dennstaedtiaceae}

\section{Pteridium Gled. Ex Scop.}

5. Pteridium aquilinum (L.) Kuhn - Kartal eğreltisi

Köprüyan1 district, degraded spruce forest, 1017 m, 08.07.2008, Uzun 1273 (KATO: 17485).

\section{F5 [5]. Cystopteridaceae}

\section{Cystopteris Bernh.}

6. Cystopteris fragilis (L.) Bernh.

Ormanüstü village, in forest, $1691 \mathrm{~m}$, 16.07.2008, Uzun 1572 (KATO: 17500).

\section{F6 [6]. Aspleniaceae}

\section{Asplenium L.}

7. Asplenium trichomanes L. subsp. trichomanes
Ormanüstü village, in forest, $1479 \mathrm{~m}$, 12.07.2008, Uzun 1441 (KATO: 17488).

8. Asplenium adiantum-nigrum L. subsp. adiantum-nigrum - Baldırıkara

Sukenar1 district, in forest, $663 \mathrm{~m}$, 07.07.2008, Uzun 1117 (KATO: 17489); Ormanüstü village, in forest, $1479 \mathrm{~m}$, 12.07.2008, Uzun 1442 (KATO: 17490); Çeşmeler district, in forest, $529 \mathrm{~m}$, 14.07.2008, Uzun 1454 (KATO: 17491); Çatak, afforestation site, $873 \mathrm{~m}, 06.08 .2008$, Uzun 1813 (KATO: 17492).

*9. Asplenium cuneifolium L.

Ormanüstü village, in forest, $1360 \mathrm{~m}$, 10.07.2008, Uzun 1361 (KATO: 17493); Çatak, afforestation site, $873 \mathrm{~m}, 06.08 .2008$, Uzun 1814 (KATO: 17494).

10. Asplenium scolopendrium L. (syn.: Phyllitis scolopendrium (L.) Newman) Geyikdili eğreltisi

Ormanüstü village, damp places in forest, $1350 \mathrm{~m}, 10.05 .2005$, Uzun 557 (KATO: 17495).

\section{F7 [7]. Athyriaceae}

\section{Athyrium Roth}

11. Athyrium filix-femina (L.) Roth

Köprüyan1 district, in forest, $977 \mathrm{~m}$, 11.07.2008, Uzun 1403 (KATO: 17496); Ormanüstü village, in forest, $1647 \mathrm{~m}$, 15.07.2008, Uzun 1537 (KATO: 17497); ibid., $1098 \mathrm{~m}, 24.07 .2008$, Uzun 1728 (KATO: 17498).

12. Athyrium distentifolium Tausch ex Opiz (syn.: Athyrium alpestre (Hoppe) T.Moore) Ormanüstü village, open forest, $1639 \mathrm{~m}$, 15.07.2008, Uzun 1538 (KATO: 17499).

\section{F8 [8]. Thelypteridaceae}

\section{Phegopteris (C. Presl) Fée}

13. Phegopteris connectilis (Michx) Watt (syn.: Thelypteris phegopteris (L.) Sloss.) Ormanüstü village, in forest, $1085 \mathrm{~m}$, 10.07.2008, Uzun 1360 (KATO: 17487).

\section{Oreopteris Holub}

14. Oreopteris limbosperma (All.) Holub (syn.: Thelypteris limbosperma (All.) H.P.Fuchs)

Köprüyanı district, in forest, $1204 \mathrm{~m}$, 24.07.2008, Uzun 1727 (KATO: 17486). 


\section{F9 [9]. Dryopteridaceae}

10. Dryopteris Adans. - Eğrelti otu

15. Dryopteris filix-mas (L.) Schott - Solucan eğreltisi

Ormanüstü village, in forest, $1085 \mathrm{~m}$, 10.07.2008, Uzun 1365 (KATO: 17512); ibid., 1676 m, 17.07.2008, Uzun 1589 (KATO: 17517); Çamlıdüz plateau, in forest, $1740 \mathrm{~m}, 22.07 .2008$, Uzun 1705 (KATO: 17519); Ormanüstü village, in forest, $1098 \mathrm{~m}$, 24.07.2008, Uzun 1730 (KATO: 17520); Köprüyanı district, in forest, $1204 \mathrm{~m}$, 24.07.2008, Uzun 1731 (KATO: 17521).

16. Dryopteris oreades Fomin

Ormanüstü village, forest edge, $1650 \mathrm{~m}$, 08.07.2008, Uzun 1274 (KATO: 17522). Euro-Sib. El.

17. Dryopteris dilatata (Hoffm.) A.Gray

Ormanüstü village, in forest, $1467 \mathrm{~m}$, 12.07.2008, Uzun 1443 (KATO: 17523). Euro-Sib. El.

*18. Dryopteris aemula (Aiton) Kuntze (syn.: Dryopteris 2iliana Golicin)

Köprüyan1 district, in forest, $977 \mathrm{~m}$, 11.07.2008, Uzun 1406 (KATO: 17524). Eux. El.

19. Dryopteris carthusiana (Vill.) H.P. Fuchs Ormanüstü village, in forest, $1236 \mathrm{~m}$, 09.07.2008, Uzun 1335 (KATO: 17525); ibid., $1280 \mathrm{~m}, 09.07 .2008$, Uzun 1336 (KATO: 17526); ibid., 1360 m, 10.07.2008, Uzun 1366 (KATO: 17527); Ormanüstü village, in forest, $1642 \mathrm{~m}, 15.07 .2008$, Uzun 1540 (KATO: 17528); ibid., $1647 \mathrm{~m}$, 15.07.2008, Uzun 1541 (KATO: 17529).

\section{Polystichum Roth}

20. Polystichum aculeatum (L.) Roth Ormanüstü village, in forest, $1085 \mathrm{~m}$, 10.07.2008, Uzun 1362 (KATO: 17501); Ormanüstü village, afforestation site, 1683 m, 10.07.2008, Uzun 1363 (KATO: 17502); ibid., $1664 \mathrm{~m}, 21.07 .2008$, Uzun 1681 (KATO: 17505); Ormanüstü village, in forest, $1948 \mathrm{~m}, 22.07 .2008$, Uzun 1704 (KATO: 17506); Ormanüstü village, in forest, $1205 \mathrm{~m}, 24.07 .2008$, Uzun 1729 (KATO: 17507).

21. Polystichum setiferum (Forssk.) T. Moore ex Woyn.- Dere gülü

Ormanüstü village, coniferous-broadleaf mixed forests, $1172 \mathrm{~m}, 10.07 .2008$, Uzun
1364 (KATO: 17508); Köprüyanı district, in forest, $997 \mathrm{~m}$, 11.07.2008, Uzun 1404 (KATO: 17509); ibid., $951 \mathrm{~m}, 11.07 .2008$, Uzun 1405 (KATO: 17510); Ormanüstü village, in forest, $1642 \mathrm{~m}, 15.07 .2008$, Uzun 1539 (KATO: 17511).

\section{F10 [10]. Polypodiaceae}

\section{Polypodium L.}

22. Polypodium vulgare $\mathrm{L}$.

Ormanüstü village, in forest, $1615 \mathrm{~m}$, 26.07.2006, Uzun 933 (KATO: 17530); Ormanüstü village, in forest, $1205 \mathrm{~m}$, 24.07.2008, Uzun 1732 (KATO: 17531).

\section{GYMNOSPERMS}

Pinales

F11 [1]. Pinaceae

\section{Picea Dietr. - Ladin}

23. Picea orientalis (L.) Peterm. - Doğu Ladini

Ormanüstü village, in forest, $1400 \mathrm{~m}$, 10.05.2005, Uzun 558 (KATO: 17532). Eux. El.

\section{Abies Mill. - Göknar}

24. Abies nordmanniana (Steven) Spach subsp. nordmanniana - Doğu Karadeniz Göknarı

Ormanüstü village, in forest, $1800 \mathrm{~m}$, 10.05.2005, Uzun 559 (KATO: 17533). Eux. El.

\section{Pinus L. - Çam}

25. Pinus sylvestris L.- Sarıçam

Köprüyanı village, steep cliffs, $900 \mathrm{~m}$, 10.05.2005, Uzun 560 (KATO: 17534). Euro-Sib. El.

\section{Cupressales}

\section{F12 [2]. Taxaceae}

\section{Taxus L.}

26. Taxus baccata L. - Yaygin porsuk Ormanüstü village, grassy place, $1162 \mathrm{~m}$, 09.07.2008, Uzun 1337 (KATO: 17535); ibid. in forest, $1622 \mathrm{~m}, 15.07 .2008$, Uzun 1542 (KATO: 17536). 


\section{F13 [3]. Cupressaceae}

\section{Juniperus L. - Ardıc}

27. Juniperus communis L. var. saxatilis Pall.

- Yaygın ardıç

Ormanüstü plateau, timberline, $2000 \mathrm{~m}$, 22.07.2008, Uzun 1706 (KATO: 17537).

28. Juniperus deltoides R.P.Adams. (syn.: Juniperus oxycedrus L. subsp. oxycedrus var. oxycedrus) - Katran ardic1

Çatak district, roadside slope, $600 \mathrm{~m}$, 11.07.2007, Uzun 968 (KATO: 17538).

\section{ANGIOSPERMS \\ MAGNOLIIDS}

Piperales Bercht. \& J.Presl

F14 [1]. Aristolochiaceae Juss.

18. Aristolochia L.

29. Aristolochia pontica Lam.

Ormanüstü village, in forest, $1067 \mathrm{~m}$, 24.07.2008, Uzun 1733 (KATO: 17539). Eux. el.

\section{MONOCOTS}

Alismatales R.Br. ex Bercht. \& J.Presl

F15 [1]. Araceae Juss.

19. Arum L. - Yılan yastığı

30. Arum maculatum L.

Ormanüstü village, damp place, $950 \mathrm{~m}$, 05.08.2005, Uzun 836 (KATO: 18555).

Dioscoreales Mart.

F16 [2]. Dioscoreaceae R.Br.

\section{Dioscorea L.}

31. Dioscorea communis (L.) Caddick \& Wilkin (syn.: Tamus communis L. subsp. communis) - Dövülmüş avrat otu

Ormanüstü village, shrubby place, $929 \mathrm{~m}$, 08.07.2005, Uzun 757 (KATO: 18757); Çeşmeler district, in forest, $460 \mathrm{~m}$, 05.08.2005, Uzun 853 (KATO: 18758); Köprüyanı district, in forest, $1001 \mathrm{~m}$, 08.07.2008, Uzun 1331 (KATO: 18759).

Liliales Perleb

F17 [3]. Melanthiaceae Batsch ex Borkh.

21. Veratrum L.

32. Veratrum album L. - Ak çöpleme
Ormanüstü plateau, open place, $1850 \mathrm{~m}$, 10.05.2005, Uzun 615 (KATO: 18749). Euro-Sib. el.

\section{Paris L.}

33. Paris incompleta M.Bieb. - Tilki üzümü Ormanüstü village, in forest, $1280 \mathrm{~m}$, 09.07.2008, Uzun 1359 (KATO: 18752); ibid., $1642 \mathrm{~m}, 15.07 .2008$, Uzun 1568 (KATO: 18753). Eux. el.

\section{F18 [4]. Colchicaceae DC.}

\section{Colchicum L. - Acı çiğdem}

34. Colchicum szovitsii Fisch. \& C.A.Mey. subsp. szovitsii

Ormanüstü plateau, melting snow patches, 1960 m, 10.05.2005, Uzun 616 (KATO: 18750). Ir.-Tur. el.

35. Colchicum speciosum Steven - Vargit çiçeği

Köprüyan1, open forest, 920 m, 23.05.2006, Uzun 893 (KATO: 18751). Hyr.-Eux. el.

\section{F19 [5]. Smilacaceae Vent.}

\section{Smilax L.}

36. Smilax excelsa L. - Gicir otu

Çatak, on bushes, $860 \mathrm{~m}, 08.07 .2008$, Uzun 1330 (KATO: 18756). Eux. el.

\section{F20 [6]. Liliaceae Juss.}

\section{Lilium L. - Zambak}

37. Lilium ciliatum P.H.Davis

Ormanüstü village, forest edge, $1596 \mathrm{~m}$, 17.07.2008, Uzun 1626 (KATO: 18742). Eux. el., Endemic, IUCN: NT previously, revised by İkinci (2005) as EN

38. Lilium armenum (Miscz. ex Grossh.) Manden (syn.: Lilium monadelphum M.Bieb. var. armenum (Miscz. ex Grossh.) P.H.Davis \& D.M.Hend.)

Çeşmeler district, grassy place, $650 \mathrm{~m}$, 01.06.2008, Uzun 1089 (KATO: 18743); Ormanüstü village, grassy place, $1162 \mathrm{~m}$, 09.07.2008, Uzun 1357 (KATO: 18744); ibid., forest edge, $1280 \mathrm{~m}, 09.07 .2008$, Uzun 1358 (KATO: 18745); ibid., $1450 \mathrm{~m}$, 10.07.2008, Uzun 1400 (KATO: 18746); ibid., $1683 \mathrm{~m}$, 10.07.2008, Uzun 1401 (KATO: 18747). Rare, IUCN: VU previously, revised by İkinci (2005) as EN 
26. Gagea Salisb.

39. Gagea bohemica (Zauschn.) Schult. \& Schult.f. (syn.: Gagea fistulosa (Ramond ex DC.) Ker Gawl.)

Ormanüstü plateau, grassy place, $1980 \mathrm{~m}$, 10.05.2005, Uzun 614 (KATO: 18748). Ir.Tur. el.

Asparagales Link

F21 [7]. Orchidaceae Juss.

\section{Neottia Guettard}

40. Neottia nidus-avis (L.) Rich. Ormanüstü village, in forest, $1479 \mathrm{~m}$, 12.07.2008, Uzun 1452 (KATO: 18760); ibid., 1627 m, 15.07.2008, Uzun 1569 (KATO: 18761). Euro-Sib. el.

\section{Cephalanthera L.C.M.Richard}

41. Cephalanthera longifolia (L.) Fritsch

Sukenar1 district, stony hill, $663 \mathrm{~m}$, 07.07.2008, Uzun 1269 (KATO: 18762); Köprüyan1 district, open forest, $1001 \mathrm{~m}$, 08.07.2008, Uzun 1332 (KATO: 18763); Çeşmeler district, in forest, $529 \mathrm{~m}$, 14.07.2008, Uzun 1533 (KATO: 187649). Euro-Sib. el.

42. Cephalanthera damasonium (Mill.) Druce.

Çatak, in forest, $483 \mathrm{~m}, 14.07 .2008$, Uzun 1534 (KATO: 18765); Çatak, in forest, 1010 m, 14.07.2008, Uzun 1535 (KATO: 18766); Çatak, in forest, $928 \mathrm{~m}, 18.07 .2008$, Uzun 1635 (KATO: 18767); Ormanüstü village, in forest, $1639 \mathrm{~m}, 27.07 .2008$, Uzun 1754 (KATO: 18768). Euro-Sib. el.

\section{Epipactis Zinn}

43. Epipactis turcica Kreutz (syn.: Epipactis tremolsii Pau subsp. turcica (Kreutz) Kreutz) Çeşmeler district, stony hill, $942 \mathrm{~m}$, 08.07.2008, Uzun 1333 (KATO: 18769). Endemic, IUCN: LC

\section{Limodorum Boehm.}

44. Limodorum abortivum (L.) Sw. var. abortivum

Çeşmeler district, Carpinus orientalis thicket, $750 \mathrm{~m}$, 01.06.2008, Uzun 1090 (KATO: 18770); Sukenarı district, stony hill, $663 \mathrm{~m}, 07.07 .2008$, Uzun 1270 (KATO: 18771); Köprüyanı district, in forest, $1001 \mathrm{~m}$, 08.07.2008, Uzun 1334 (KATO: 18772);
Çatak, in forest, $940 \mathrm{~m}, 18.07 .2008$, Uzun 1636 (KATO: 18773).

\section{Goodyera R.Br.}

45. Goodyera repens (L.) R.Br.

Ormanüstü village, in spruce forest, $1615 \mathrm{~m}$, 26.07.2006, Uzun 966 (KATO: 18774); ibid., $1479 \mathrm{~m}, 12.07 .2008$, Uzun 1453 (KATO: 18775); ibid., $1622 \mathrm{~m}$, 15.07.2008, Uzun 1570 (KATO: 18776); Ormanüstü plateau, in forest, $1615 \mathrm{~m}, 17.07 .2008$, Uzun 1627 (KATO: 18777); Ormanüstü village, in forest, $1710 \mathrm{~m}, 21.07 .2008$, Uzun 1703 (KATO: 18778). Euro-Sib. el.

\section{Gymnadenia R.Br.}

46. Gymnadenia conopsea (L.) R.Br. Ormanüstü plateau, grassy place, $1900 \mathrm{~m}$, 08.07.2005, Uzun 759 (KATO: 18779); ibid., $1850 \mathrm{~m}, 05.08 .2005$, Uzun 854 (KATO: 18780). Euro-Sib. el.

\section{Platanthera Rich.}

47. Platanthera chlorantha (Custer) Rchb. Ormanüstü village, in forest, $1014 \mathrm{~m}$, 11.07.2008, Uzun 1440 (KATO: 18781); Ormanüstü village, open forest, $1639 \mathrm{~m}$, 15.07.2008, Uzun 1571 (KATO: 18782).

\section{Ophrys L. - Kazankarası}

48. Ophrys sphegodes Mill. subsp. sphegodes Çeşmeler district, grassy place, $700 \mathrm{~m}$, 01.06.2008, Uzun 1091 (KATO: 18783).

49. Ophrys oestrifera M.Bieb. subsp. oestrifera

Çeşmeler district, grassy place, $700 \mathrm{~m}$, 01.06.2008, Uzun 1092 (KATO: 18784).

\section{Anacamptis Rich.}

50. Anacamptis pyramidalis (L.) Rich. - Çam salebi

Yukarıköy village, afforestation site, $836 \mathrm{~m}$, 18.07.2008, Uzun 1637 (KATO: 18785).

\section{Neotinea Rchb.f. - Salep}

51. Neotinea tridentata (Scop.) R.M.Bateman, Pridgeon \& M.W.Chase (syn.: Orchis tridentata Scop.) - Beyaz dağ salebi

Ormanüstü village, open hills, $1300 \mathrm{~m}$, 08.07.2005, Uzun 760 (KATO: 18786); Çeşmeler district, stony hills, $634 \mathrm{~m}$, 07.07.2008, Uzun 1271 (KATO: 18787); 
Çatak, open forest, $458 \mathrm{~m}, 14.07 .2008$, Uzun 1536 (KATO: 18788). Medit. el.

\section{Orchis L. - Salep}

52. Orchis simia Lam. - Püsküllü, Tavşantopuğu

Çeşmeler district, roadside slope, $500 \mathrm{~m}$, 10.05.2005, Uzun 617 (KATO: 18789); ibid., grassy place, $700 \mathrm{~m}, 01.06 .2008$, Uzun 1093 (KATO: 18790). Medit. el.

\section{Dactylorhiza Neck. ex Nevski}

53. Dactylorhiza urvilleana (Steud.) H.Baumann \& Künkele subsp. urvilleana Ormanüstü plateau, afforestation site, 1862 m, 02.08.2008, Uzun 1812 (KATO: 18791). Eux. el.

54. Dactylorhiza euxina (Nevski) Czerep. subsp. euxina var. euxina

Çamlıdüz plateau, afforestation site, $1728 \mathrm{~m}$, 20.07.2008, Uzun 1680 (KATO: 18792). Eux. el.

\section{F22 [8]. Iridaceae Juss.}

\section{Crocus Herb. - Çiğdem}

55. Crocus vallicola Herb.

Ormanüstü village, grassy place, $1300 \mathrm{~m}$, 09.09.2007, Uzun 1049 (KATO: 18755). Eux. el.

\section{F23 [9]. Asphodelaceae Juss.}

40. Asphodeline Rchb. - Çiriş otu, Dede değneği

56. Asphodeline lutea (L.) Rchb.

Çeşmeler district, shrubby place, $600 \mathrm{~m}$, 01.06.2008, Uzun 1087 (KATO: 18731).

Medit. el.

\section{F24 [10]. Amaryllidaceae J.St.-Hil.}

\section{Galanthus L. - Kardelen}

57. Galanthus rizehensis Stern

Köprüyan1, open forest, 920 m, 23.04.2006, Uzun 858 (KATO: 18754). Eux. el., Rare, IUCN: VU

\section{F25 [11]. Asparagaceae Juss.}

\section{Ruscus L.}

58. Ruscus aculeatus L. (syn.: Ruscus aculeatus L. var. angustifolius Boiss) Tavşan kirazı
Çeşmeler district, stony hill, $634 \mathrm{~m}$, 07.07.2008, Uzun 1267 (KATO: 18724).

59. Ruscus colchicus Yeo-Zirmek

Köprüyan1 district, in forest, $1067 \mathrm{~m}$, 24.07.2008, Uzun 1748 (KATO: 18725).

Eux. el., Rare, IUCN: VU

43. Polygonatum Mill. - Mührü Süleyman

60. Polygonatum verticillatum (L.) All.

Ormanüstü plateau, open land, $1596 \mathrm{~m}$, 17.07.2008, Uzun 1625 (KATO: 18726). Euro-Sib. el.

61. Polygonatum multiflorum (L.) All

Ormanüstü village, spruce forest, $1150 \mathrm{~m}$, 05.08.2005, Uzun 851 (KATO: 18727); Köprüyanı district, shrubby place, $1100 \mathrm{~m}$, 23.05.2006, Uzun 890 (KATO: 18728); Çeşmeler district, in forest, $529 \mathrm{~m}$, 14.07.2008, Uzun 1532 (KATO: 18729); Çatak, in forest, $873 \mathrm{~m}, 18.07 .2008$, Uzun 1634 (KATO: 18730).

\section{Scilla L. - Mavi yıldız}

62. Scilla monanthos K.Koch

Ormanüstü plateau, damp meadows, $1720 \mathrm{~m}$, 10.05.2005, Uzun 611 (KATO: 18732). Eux. el.

63. Scilla siberica Haw. subsp. armena (Grossh.) Mordak)

Ormanüstü village, damp meadows, $1700 \mathrm{~m}$, 10.05.2005, Uzun 612 (KATO: 18733). Ir.Tur. el.

\section{Ornithogalum L. - Ak yıldız, Tükrük otu}

64. Ornithogalum narbonense L. - Ak baldır Çeşmeler district, grassy place, $700 \mathrm{~m}$, 01.06.2008, Uzun 1088 (KATO: 18734). Medit. el.

65. Ornithogalum oligophyllum E.D. Clarke - Kurt soğanı

Ormanüstü plateau, alpine hill, $2000 \mathrm{~m}$, 08.07.2005, Uzun 756 (KATO: 18735); ibid., wet hill, $1900 \mathrm{~m}, 05.08 .2005$, Uzun 852 (KATO: 18736).

\section{Muscari Mill. - Arap sümbülü}

66. Muscari armeniacum Leichtlin ex Baker Ormanüstü Yaylası, grassy place, $1800 \mathrm{~m}$, 10.05.2005, Uzun 613 (KATO: 18737); Çeşmeler district, stony places, $583 \mathrm{~m}$, 07.07.2008, Uzun 1268 (KATO: 18738); 
ibid., 942 m, 08.07.2008, Uzun 1329 (KATO: 18739).

67. Muscari neglectum Guss. ex Ten.

Köprüyan1, sandy hills, 620 m, 23.05.2006, Uzun 891 (KATO: 18740); ibid., $920 \mathrm{~m}$, 23.05.2006, Uzun 892 (KATO: 18741).

Poales Small

F26 [12]. Juncaceae Juss.

\section{Juncus L. - Cayır sazı}

68. Juncus inflexus L. subsp. inflexus

Ormanüstü village, damp places, $1750 \mathrm{~m}$, 05.08.2005, Uzun 837 (KATO: 18556).

69. Juncus effusus L. subsp. effusus - Gevşek hasir otu

Ormanüstü village, damp places, $1750 \mathrm{~m}$, 05.08.2005, Uzun 838 (KATO: 18557); ibid., streamside, $1400 \mathrm{~m}, 12.07 .2007$, Uzun 1027 (KATO: 18558). Cosmopolitan

70. Juncus bufonius L.

Ormanüstü village, wet rocky hills, $1300 \mathrm{~m}$, 08.07.2005, Uzun 742 (KATO: 18559). Cosmopolitan

71. Juncus articulatus L. subsp. articulatus Ormanüstü plateau, damp meadows, $1805 \mathrm{~m}$, 05.08.2005, Uzun 839 (KATO: 18560); ibid., $1850 \mathrm{~m}, 05.08 .2005$, Uzun 840 (KATO: 18561). Euro-Sib. el.

\section{Luzula DC. - Kuzu levreği}

72. Luzula forsteri (Sm.) DC.

Ormanüstü village, roadside, $1550 \mathrm{~m}$, 10.05.2005, Uzun 601 (KATO: 18562); ibid., in forest, $1467 \mathrm{~m}, 12.07 .2008$, Uzun 1451 (KATO: 18563); ibid., open forest, $1627 \mathrm{~m}$, 15.07.2008, Uzun 1559 (KATO: 18564); ibid., $1912 \mathrm{~m}$, 16.07.2008, Uzun 1583 (KATO: 18565); ibid., in forest, $1691 \mathrm{~m}$, 16.07.2008, Uzun 1584 (KATO: 18566); Çamlıdüz plateau, in forest, $1576 \mathrm{~m}$, 17.07.2008, Uzun 1617 (KATO: 18567); Çamlıdüz plateau, in forest, $1720 \mathrm{~m}$, 17.07.2008, Uzun 1618 (KATO: 18568). Euro-Sib. el.

73. Luzula sylvatica (Huds.) Gaudin subsp. sylvatica

Ormanüstü village, in forest, $1752 \mathrm{~m}$, 21.07.2008, Uzun 1698 (KATO: 18569). Euro-Sib. el.

74. Luzula stenophylla Steud. (syn.: Luzula pseudosudetica (V.Krecz.) V.Krecz. apud V.Krecz. \& Gontsh.)
Çamlıdüz plateau, in forest, $1769 \mathrm{~m}$, 22.07.2008, Uzun 1723 (KATO: 18570).

Eux. (mt.) el.

75. Luzula multiflora (Ehrh.) Lej. subsp. multiflora

Ormanüstü plateau, grassy place, $2014 \mathrm{~m}$, 08.07.2005, Uzun 743 (KATO: 18571); ibid., $2000 \mathrm{~m}$, 05.08.2005, Uzun 841 (KATO: 18572).

\section{F27 [13]. Cyperaceae Juss.}

\section{Carex L. - Ekşi çimen, Ayak otu}

76. Carex leersii F.W.Schultz (syn.: Carex divulsa Stokes subsp. leersii (F.W.Schultz) W.Koch)

Çeşmeler district, stony hills, $634 \mathrm{~m}$, 07.07.2008, Uzun 1253 (KATO: 18573); Sukenar1 district, in forest, $498 \mathrm{~m}$, 11.07.2008, Uzun 1435 (KATO: 18574). Euro-Sib. el.

77. Carex muricata L.

Ormanüstü plateau, grassy place, $2000 \mathrm{~m}$, 16.07.2008, Uzun 1585 (KATO: 18575). Euro-Sib. el.

78. Carex pendula Huds.

Ormanüstü village, forest edge, damp place, 1280 m, 09.07.2008, Uzun 1353 (KATO: 18576). Euro-Sib. el.

79. Carex sylvatica Huds. subsp. sylvatica Ormanüstü village, wet hills, $1750 \mathrm{~m}$, 05.08.2005, Uzun 842 (KATO: 18577); ibid., grassy place, $1162 \mathrm{~m}, 09.07 .2008$, Uzun 1354 (KATO: 18578); ibid., in forest, $1642 \mathrm{~m}$, 15.07.2008, Uzun 1560 (KATO: 18579); Ormanüstü village, open forest, $1627 \mathrm{~m}$, 15.07.2008, Uzun 1561 (KATO: 18580); ibid., $1639 \mathrm{~m}$, 15.07.2008, Uzun 1562 (KATO: 18581); Ormanüstü plateau, in forest, $1668 \mathrm{~m}$, 17.07.2008, Uzun 1619 (KATO: 18582); ibid., 1615 m, 17.07.2008, Uzun 1620 (KATO: 18583); Çamlıdüz plateau, in forest, $1720 \mathrm{~m}, 17.07 .2008$, Uzun 1621 (KATO: 18584); Ormanüstü plateau, afforestation site, $1664 \mathrm{~m}, 21.07 .2008$, Uzun 1699 (KATO: 18585); Ormanüstü village, in forest, $1745 \mathrm{~m}, 21.07 .2008$, Uzun 1700 (KATO: 18586); Ormanüstü plateau, afforestation site, $1862 \mathrm{~m}, 02.08 .2008$, Uzun 1796 (KATO: 18587). Euro-Sib. el.

*80. Carex flacca Schreb. subsp. flacca Ormanüstü plateau, grassy place, $1800 \mathrm{~m}$, 18.07.2008, Uzun 1633 (KATO: 18588). 
81. Carex flacca Schreb. subsp. erythrostachys (Hoppe) Holub (syn.: Carex flacca Schreb. subsp. serratula (Biv. ex Spreng.) Greuter)

Çatak, in forest, $483 \mathrm{~m}, 14.07 .2008$, Uzun 1512 (KATO: 18589). Medit. el.

82. Carex pallescens L. (syn.: Carex pallescens L. var. chalcodeta (V.Krecz.) Ö.Nilsson)

Ormanüstü plateau, wet meadows, $2000 \mathrm{~m}$, 05.08.2005, Uzun 843 (KATO: 18590). Eux. el.

83. Carex digitata $\mathrm{L}$.

Ormanüstü village, in forest, $1945 \mathrm{~m}$, 16.07.2008, Uzun 1586 (KATO: 18591); Ormanüstü plateau, pure Picea orientalis stand, $1912 \mathrm{~m}$, 16.07.2008, Uzun 1587 (KATO: 18592). Eux. el.

84. Carex depressa Link subsp. transsilvanica (Schur) K.Richt.

Ormanüstü plateau, grassy place, $2000 \mathrm{~m}$, 08.07.2005, Uzun 744 (KATO: 18593); Çeşmeler district, stony hills, $490 \mathrm{~m}$, 07.07.2008, Uzun 1254 (KATO: 18594). Euro-Sib. el.

85. Carex liparocarpos Gaudin subsp. bordzilowskii (V.I.Krecz.) T.V. Egorova

Çatak, open forest, $458 \mathrm{~m}, 14.07 .2008$, Uzun 1513 (KATO: 18595). Hyr.-Eux. el.

\section{F28 [14]. Poaceae Barnhart (= Gramineae} Juss.)

\section{Brachypodium L.}

86. Brachypodium distachyon (L.) P.Beauv. (syn.: Trachynia distachya (L.) Link)

Çeşmeler, damp place, $437 \mathrm{~m}, 07.07 .2008$, Uzun 1258 (KATO: 18616).

87. Brachypodium pinnatum (L.) P.Beauv. Ceșmeler district, stony hills, $490 \mathrm{~m}$, 07.07.2008, Uzun 1256 (KATO: 18605); Sukenar1 district, in forest, $663 \mathrm{~m}$, 07.07.2008, Uzun 1257 (KATO: 18608); Köprüyanı district, rocky place, $860 \mathrm{~m}$, 08.07.2008, Uzun 1326 (KATO: 18609); Çatak, afforestation site, $477 \mathrm{~m}, 14.07 .2008$, Uzun 1515 (KATO: 18613); Yukarıköy village, in forest, $931 \mathrm{~m}, 14.07 .2008$, Uzun 1516 (KATO: 18614); Çatak, afforestation site, 873 m, 06.08.2008, Uzun 1837 (KATO: 18615). Euro-Sib. el.

88. Brachypodium sylvaticum (Huds.) P.Beauv. subsp. sylvaticum
Yenimahalle, forest edge, $780 \mathrm{~m}, 26.07 .2006$, Uzun 965 (KATO: 18596); Kiremitli district, grassy place, $717 \mathrm{~m}, 13.07 .2007$, Uzun 1047 (KATO: 18597); Sukenarı district, in forest, $663 \mathrm{~m}, 07.07 .2008$, Uzun 1255 (KATO: 18598); Köprüyanı district, open forest, 701 m, 08.07.2008, Uzun 1325 (KATO: 18599); Çatak, in forest, $483 \mathrm{~m}, 14.07 .2008$, Uzun 1514 (KATO: 18601); Çeşmeler district, degraded spruce forest, $541 \mathrm{~m}, 06.08 .2008$, Uzun 1836 (KATO: 18604). Euro-Sib. el.

\section{Elymus L.}

89. Elymus repens (L.) Gould subsp. repens - Ayrik otu, Demir otu

Ormanüstü village, grassy place, $850 \mathrm{~m}$, 14.07.2008, Uzun 1517 (KATO: 18617).

\section{Hordeum L. - Arpa}

*90. Hordeum bulbosum L.

Çatak, roadside, $400 \mathrm{~m}, 11.07 .2008$, Uzun 1436 (KATO: 18618); Yukarıköy village, grassy place, $850 \mathrm{~m}, 14.07 .2008$, Uzun 1518 (KATO: 18619).

\section{Bromus L. - Brom}

91. Bromus racemosus L. subsp. racemosus Yukarıköy village, grassy place, $850 \mathrm{~m}$, 14.07.2008, Uzun 1519 (KATO: 18620). Euro-Sib. el.

92. Bromus commutatus Schrad. subsp. commutatus

Ormanüstü village, grassy place, $1000 \mathrm{~m}$, 10.05.2005, Uzun 602 (KATO: 18621).

93. Bromus madritensis L. subsp. madritensis Çatak, open forest, $458 \mathrm{~m}, 14.07 .2008$, Uzun 1520 (KATO: 18622).

*94. Bromus benekenii (Lange) Trimen Ormanüstü village, open forest, $1627 \mathrm{~m}$, 15.07.2008, Uzun 1563 (KATO: 18623); ibid., $1639 \mathrm{~m}$, 15.07.2008, Uzun 1564 (KATO: 18624); Ormanüstü village, in forest, $1205 \mathrm{~m}, 24.07 .2008$, Uzun 1747 (KATO: 18625).

\section{Avena L. - Yulaf}

*95. Avena fatua L. subsp. fatua

Çeşmeler district, roadside, $650 \mathrm{~m}$, 08.07.2005, Uzun 745 (KATO: 18626).

96. Avena sterilis L. subsp. ludoviciana (Durieu) Gillet \& Magne

Çeşmeler district, roadside, $500 \mathrm{~m}$, 10.05.2005, Uzun 603 (KATO: 18627). 


\section{Helictochloa Romero Zarco}

97. Helictochloa planiculmis (Schrad.) Romero Zarco (syn.: Helictotrichon planiculme (Schrad.) Pilg.)

Ormanüstü plateau, grassy place, $2000 \mathrm{~m}$, 05.06.2006, Uzun 930 (KATO: 18628); Ormanüstü plateau, degraded spruce forest, 1991 m, 02.08.2008, Uzun 1797 (KATO: 18629); Ormanüstü plateau, stony degraded spruce forest, $2000 \mathrm{~m}, 02.08 .2008$, Uzun 1798 (KATO: 18630). Eux. el.

\section{Gaudiniopsis Eig}

98. Gaudiniopsis macra (M.Bieb.) Eig subsp. macra

Ormanüstü plateau, grassy place, $2014 \mathrm{~m}$, 08.07.2005, Uzun 746 (KATO: 18631). Ir.Tur. el.

\section{Trisetum Pers.}

99. Trisetum flavescens (L.) P.Beauv. subsp. flavescens

Ormanüstü plateau, grassy place, $2020 \mathrm{~m}$, 05.08.2005, Uzun 844 (KATO: 18632). Euro-Sib. el.

\section{Holcus L.}

100. Holcus lanatus L. subsp. lanatus

Ormanüstü village, moist grassy place, 1000 m, 10.05.2005, Uzun 604 (KATO: 18633). Euro-Sib. el.

\section{Calamagrostis Adanson}

101. Calamagrostis arundinacea (L.) Roth. Ormanüstü village, afforestation site, 1683 m, 10.07.2008, Uzun 1393 (KATO: 18634); ibid., in forest, $1622 \mathrm{~m}, 15.07 .2008$, Uzun 1565 (KATO: 18635); Ormanüstü plateau, in forest, $1738 \mathrm{~m}$, 16.07.2008, Uzun 1588 (KATO: 18636); Camlidüz plateau, in forest, 1740 m, 22.07.2008, Uzun 1724 (KATO: 18637); Ormanüstü plateau, degraded spruce forest, $1991 \mathrm{~m}$, 02.08.2008, Uzun 1799 (KATO: 18638). Euro-Sib. el.

\section{Agrostis L.}

102. Agrostis gigantea Roth subsp. gigantea Ormanüstü village, moist grassy place, 1000 m, 10.05.2005, Uzun 605 (KATO: 18639); Ormanüstü plateau, grassy place, $2014 \mathrm{~m}$, 08.07.2005, Uzun 747 (KATO: 18640); Ormanüstü village, grassy place, $1280 \mathrm{~m}$, 09.07.2008, Uzun 1355 (KATO: 18641);
Ormanüstü village, afforestation site, 1683 m, 10.07.2008, Uzun 1394 (KATO: 18642); Çamlıdüz plateau, afforestation site, $1728 \mathrm{~m}$, 20.07.2008, Uzun 1677 (KATO: 18643); ibid., $1862 \mathrm{~m}, 02.08 .2008$, Uzun 1800 (KATO: 18644); Ormanüstü plateau, degraded spruce forest, $1991 \mathrm{~m}, 02.08 .2008$, Uzun 1801 (KATO: 18645). Euro-Sib. el.

103. Agrostis capillaris L. subsp. capillaris Ormanüstü plateau, grassy place, $1805 \mathrm{~m}$, 05.08.2005, Uzun 845 (KATO: 18646).

\section{Anthoxanthum L.}

104. Anthoxanthum odoratum L. subsp. alpinum (A.Löve \& D.Löve) B.M.G.Jones \& Melderis

Ormanüstü plateau, open forest, $1979 \mathrm{~m}$, 02.08.2008, Uzun 1802 (KATO: 18647); Ormanüstü plateau, degraded spruce forest, $1991 \mathrm{~m}, 02.08 .2008$, Uzun 1803 (KATO: 18648); Ormanüstü plateau, stony degraded spruce forest, $2000 \mathrm{~m}, 02.08 .2008$, Uzun 1804 (KATO: 18649). Euro-Sib. el.

\section{Phleum L. - Çayır köpek kuyruğu}

105. Phleum nodosum L. (syn.: Phleum bertolonii DC.)

Çatak, afforestation site, $873 \mathrm{~m}, 06.08 .2008$, Uzun 1838 (KATO: 18650).

106. Phleum montanum K.Koch subsp. montanum

Çeşmeler district, stony hills, $634 \mathrm{~m}$, 07.07.2008, Uzun 1259 (KATO: 18651).

\section{Festuca L. - Çayır yumağı}

107. Festuca airoides Lam.

Ormanüstü plateau, grassy place, $2014 \mathrm{~m}$, 08.07.2005, Uzun 748 (KATO: 18662); ibid., 2000 m, 05.08.2005, Uzun 846 (KATO: 18663); ibid., 2000 m, 05.06.2006, Uzun 931 (KATO: 18664); Çamlıdüz plateau, afforestation site, $1728 \mathrm{~m}, 20.07 .2008$, Uzun 1678 (KATO: 18665); Ormanüstü plateau, degraded spruce forest, $1991 \mathrm{~m}, 02.08 .2008$, Uzun 1806 (KATO: 18666). Euro-Sib. (mt.) el.

108. Festuca heterophylla Lam.

Köprüyanı district, open forest, $975 \mathrm{~m}$, 08.07.2008, Uzun 1327 (KATO: 18667); Çeşmeler district, stony hill, $632 \mathrm{~m}$, 11.07.2008, Uzun 1437 (KATO: 18668); Çatak, open forest, $458 \mathrm{~m}, 14.07 .2008$, Uzun 1523 (KATO: 18669). Euro-Sib. el. 
109. Festuca chalcophaea V.I.Krecz. \& Bobrov subsp. euryphylla (St.-Yves) Markgr.-Dann.

Ormanüstü village, afforestation site, 1683 m, 10.07.2008, Uzun 1396 (KATO: 18670). Ir.-Tur. el.

110. Festuca amethystina L. subsp. orientalis Krajina var. turcica Markgr.-Dann.

Ormanüstü plateau, stony degraded spruce forest, $2000 \mathrm{~m}, 02.08 .2008$, Uzun 1807 (KATO: 18671). Euro-Sib. (mt.) el., Endemic, IUCN: NT

\section{Drymochloa Holub.}

111. Drymochloa drymeja (Mert. \& W. D. J. Koch) Holub subsp. drymeja (bas.: Festuca drymeja Mert. \& W.D.J.Koch)

Ormanüstü village, in forest, $1236 \mathrm{~m}$, 09.07.2008, Uzun 1356 (KATO: 18652); Ormanüstü village, coniferous-broadleaf mixed forests, $1172 \mathrm{~m}, 10.07 .2008$, Uzun 1395 (KATO: 18653); Çeşmeler district, in forest, $529 \mathrm{~m}, 14.07 .2008$, Uzun 1521 (KATO: 18654); Ormanüstü village, open forest, $1627 \mathrm{~m}$, 15.07.2008, Uzun 1566 (KATO: 18655); Ormanüstü plateau, in forest, $1668 \mathrm{~m}$, 17.07.2008, Uzun 1622 (KATO: 18656); ibid., 1615 m, 17.07.2008, Uzun 1623 (KATO: 18657); Ormanüstü plateau, afforestation site, $1664 \mathrm{~m}$, 21.07.2008, Uzun 1701 (KATO: 18658); Ormanüstü village, in forest, $1752 \mathrm{~m}$, 21.07.2008, Uzun 1702 (KATO: 18659); Ormanüstü plateau, afforestation site, 1862 m, 02.08.2008, Uzun 1805 (KATO: 18660). Euro-Sib. el.

\section{Schedonorus P.Beauv.}

*112. Schedonorus arundinaceus (Schreb.) Dumont subsp. arundinaceus (syn.: Lolium arundinaceum (Schreb.) Darbysh. subsp. arundinaceum, Festuca arundinacea Schreb. subsp. arundinacea)

Yukarıköy village, grassy place, $850 \mathrm{~m}$, 14.07.2008, Uzun 1522 (KATO: 18661).

\section{Bellardiochloa Chiov.}

113. Bellardiochloa polychroa (Trautv.) Roshev.

Ormanüstü plateau, afforestation site, 1862 m, 02.08.2008, Uzun 1808 (KATO: 18672). Ir.-Tur. el.
67. Lolium L. - İngiliz çimi

114. Lolium perenne L.

Ormanüstü village, damp grassy place, 1000 m, 10.05.2005, Uzun 606 (KATO: 18673); Ormanüstü plateau, grassy place, $2000 \mathrm{~m}$, 08.07.2005, Uzun 749 (KATO: 18674). Euro-Sib. el.

\section{Vulpia C.C.Gmelin}

115. Vulpia ciliata Dumort. subsp. ciliata

Çeşmeler, stony-grassy place, $437 \mathrm{~m}$, 07.07.2008, Uzun 1260 (KATO: 18675).

\section{Catapodium Link}

116. Catapodium rigidum (L.) C.E.Hubb. subsp. majus (C.Presl) F.H.Perring \& P.D.Sell

Çeşmeler district, stony place, $583 \mathrm{~m}$, 07.07.2008, Uzun 1261 (KATO: 18676).

\section{Poa L.}

117. Poa annua $\mathrm{L}$.

Ormanüstü village, grassy place, $1190 \mathrm{~m}$, 05.08.2005, Uzun 847 (KATO: 18677). Cosmopolitan

118. Poa trivialis L.

Ormanüstü village, damp grassy place, 1000 m, 10.05.2005, Uzun 607 (KATO: 18678); Ormanüstü village, damp grassy place, 1300 m, 08.07.2005, Uzun 608 (KATO: 18679).

119. Poa pratensis L. subsp. pratensis Ormanüstü village, open place, $929 \mathrm{~m}$, 08.07.2005, Uzun 750 (KATO: 18680); Ormanüstü village, grassy place, $1400 \mathrm{~m}$, 12.07.2007, Uzun 1028 (KATO: 18681).

120. Poa angustifolia $\mathrm{L}$.

Ormanüstü village, afforestation site, 1683 m, 10.07.2008, Uzun 1397 (KATO: 18682).

121. Poa nemoralis L. subsp. nemoralis Ormanüstü village, grassy place, $1500-1750$ $\mathrm{m}$, 08.07.2005, Uzun 751 (KATO: 18683); Sukenar1 district, in forest, $663 \mathrm{~m}$, 07.07.2008, Uzun 1262 (KATO: 18684); Ormanüstü village, coniferous-broadleaf mixed forests, $1172 \mathrm{~m}, 10.07 .2008$, Uzun 1398 (KATO: 18685); Köprüyanı district, in forest, $719 \mathrm{~m}$, 11.07.2008, Uzun 1438 (KATO: 18686); Ormanüstü village, grassy place, $1400 \mathrm{~m}, 12.07 .2007$, Uzun 1029 (KATO: 18687); Çeşmeler district, in forest, $529 \mathrm{~m}, 14.07 .2008$, Uzun 1524 (KATO: 18688); Ormanüstü village, in forest, $1627 \mathrm{~m}$, 15.07.2008, Uzun 1567 (KATO: 18689); 
Çamlıdüz plateau, in forest, $1740 \mathrm{~m}$, 22.07.2008, Uzun 1725 (KATO: 18690).

\section{Dactylis L. - Domuz ayrı̆̆ı}

122. Dactylis glomerata L. subsp. glomerata Ormanüstü village, grassy place, $1750 \mathrm{~m}$, 05.08.2005, Uzun 848 (KATO: 18691); Kiremitli district, grassy place, $717 \mathrm{~m}$, 13.07.2007, Uzun 1048 (KATO: 18692); Çeşmeler district, stony place, $583 \mathrm{~m}$, 07.07.2008, Uzun 1263 (KATO: 18693); Çatak, afforestation site, $477 \mathrm{~m}, 14.07 .2008$, Uzun 1525 (KATO: 18694); Çatak, in forest, 1010 m, 14.07.2008, Uzun 1526 (KATO: 18695). Euro-Sib. el.

123. Dactylis glomerata L. subsp. hispanica (Roth) Nyman

Ormanüstü plateau, grassy place, $1900 \mathrm{~m}$, 05.08.2005, Uzun 849 (KATO: 18696).

\section{Cynosurus $\mathrm{L}$.}

124. Cynosurus cristatus L.

Ormanüstü village, damp grassy place, 1000 m, 10.05.2005, Uzun 609 (KATO: 18697); Ormanüstü plateau, grassy place, $2000 \mathrm{~m}$, 08.07.2005, Uzun 752 (KATO: 18698); ibid., $1805 \mathrm{~m}, 05.08 .2005$, Uzun 850 (KATO: 18699); ibid., 2020 m, 05.06.2006, Uzun 932 (KATO: 18700); Ormanüstü village, afforestation site, $1683 \mathrm{~m}, 10.07 .2008$, Uzun 1399 (KATO: 18701); Çamlıdüz plateau, afforestation site, $1728 \mathrm{~m}, 20.07 .2008$, Uzun 1679 (KATO: 18702). Euro-Sib. el.

125. Cynosurus echinatus L.

Yukarıköy village, grassy place, $850 \mathrm{~m}$, 11.07.2007, Uzun 1022 (KATO: 18703); Çatak, afforestation site, $873 \mathrm{~m}, 06.08 .2008$, Uzun 1839 (KATO: 18704). Medit. el.

\section{Briza L.}

126. Briza media L.

Ormanüstü village, nemli grassy place, 1000 m, 10.05.2005, Uzun 610 (KATO: 18705); Çeşmeler district, open place, $595 \mathrm{~m}$, 08.07.2005, Uzun 753 (KATO: 18706); Ormanüstü village, open place, $929 \mathrm{~m}$, 08.07.2005, Uzun 754 (KATO: 18707); Ormanüstü plateau, grassy place, $1814 \mathrm{~m}$, 08.07.2005, Uzun 755 (KATO: 18708); Çeşmeler district, stony hills, $634 \mathrm{~m}$, 07.07.2008, Uzun 1264 (KATO: 18709); Çatak, open forest, $458 \mathrm{~m}, 14.07 .2008$, Uzun 1527 (KATO: 18710).

\section{Sesleria Scop.}

127. Sesleria alba $\mathrm{Sm}$. subsp. alba

Çeşmeler district, stony hill, $942 \mathrm{~m}$, 08.07.2008, Uzun 1328 (KATO: 18711); Çatak, open forest, $458 \mathrm{~m}, 14.07 .2008$, Uzun 1528 (KATO: 18712).

\section{Melica L.}

128. Melica uniflora Retz.

Çatak, in forest, $486 \mathrm{~m}, 14.07 .2008$, Uzun 1529 (KATO: 18713); Çeşmeler district, in forest, $529 \mathrm{~m}$, 14.07.2008, Uzun 1530 (KATO: 18714). Euro-Sib. el.

129. Melica ciliata L. subsp. ciliata

Çeşmeler district, stony-grassy hill, $1050 \mathrm{~m}$, 11.07.2007, Uzun 1023 (KATO: 18715); Yukarıköy village, grassy place, $850 \mathrm{~m}$, 14.07.2008. Uzun 1531 (KATO: 18716). Euro-Sib. el.

\section{Nardus L.}

130. Nardus stricta L.

Ormanüstü plateau, grassy place, $2000 \mathrm{~m}$, 17.07.2008., Uzun 1624 (KATO: 18717); Ormanüstü plateau, stony degraded spruce forest, $2010 \mathrm{~m}$, 02.08.2008, Uzun 1809 (KATO: 18718). Euro-Sib. el.

\section{Achnatherum P.Beauv.}

131. Achnatherum bromoides (L.) P. Beauv. (syn.: Stipa bromoides (L.) Dörfl.)

Çeşmeler district, stony hills, $490 \mathrm{~m}$, 07.07.2008, Uzun 1265 (KATO: 18719).Medit. el.

\section{Danthonia DC.}

132. Danthonia decumbens (L.) DC. subsp. decumbens

Ormanüstü plateau, forest edge, $1979 \mathrm{~m}$, 02.08.2008, Uzun 1810 (KATO: 18720); ibid., $1991 \mathrm{~m}$, 02.08.2008, Uzun 1811 (KATO: 18721).

\section{Bothriochloa O.Kuntze}

133. Bothriochloa ischaemum (L.) Keng Çeşmeler district, stony hill, $634 \mathrm{~m}$, 07.07.2008, Uzun 1266 (KATO: 18722); Çeşmeler district, stony hill, $632 \mathrm{~m}$, 11.07.2008, Uzun 1439 (KATO: 18723). 


\section{EUDICOTS}

Ranunculales Juss. ex Bercht. \& J.Presl

F29 [1]. Papaveraceae Juss.

\section{Corydalis Medik. - Kazgagası}

134. Corydalis caucasica DC. subsp. caucasica

Ormanüstü village, open forest edge, $1550 \mathrm{~m}$, 10.05.2005, Uzun 563 (KATO: 17563). Eux. el.

\section{Fumaria L. - Sahtere otu}

*135. Fumaria densiflora DC.

Çeşmeler district, grassy place, $700 \mathrm{~m}$, 01.06.2008, Uzun 1051 (KATO: 17564).

136. Fumaria officinalis L. subsp. officinalis - Şahtere

Çeşmeler district, roadside, $663 \mathrm{~m}$, 07.07.2008, Uzun 1120 (KATO: 17565).

\section{Papaver L. - Gelincik}

137. Papaver rhoeas L. subsp. rhoeas

Çeşmeler district, roadside, $600 \mathrm{~m}$, 10.06.2005, Uzun 619 (KATO: 17561).

138. Papaver dubium L. subsp. dubium

Sukenarı district, roadside slope, $663 \mathrm{~m}$, 07.07.2008, Uzun 1119 (KATO: 17562).

\section{F30 [2]. Berberidaceae Juss.}

\section{Epimedium L. - Keşiş külahı}

139. Epimedium pubigerum (DC.) Moren \& Decne.

Ormanüstü village, in forest, $663 \mathrm{~m}$, 07.07.2008, Uzun 1118 (KATO: 17560). Eux. el.

\section{F31 [3]. Ranunculaceae Juss.}

\section{Helleborus L. - Noel gülü}

140. Helleborus orientalis Lam. - Doğu noel gülü

Köprüyan1, open forest, 800 m, 23.05.2006, Uzun 859 (KATO: 17540); Köprüyan1, open hill, 920 m, 23.05.2006, Uzun 860 (KATO: 17541). Eux. el.

\section{Actaea L.}

141. Actaea spicata L. - Domuz üzümü Ormanüstü village, in forest, $1600 \mathrm{~m}$, 05.08.2005, Uzun 761 (KATO: 17542); ibid., 1642 m, 15.07.2008, Uzun 1543 (KATO:
17543); ibid., $1475 \mathrm{~m}, 21.07 .2008$, Uzun 1682 (KATO: 17544).

\section{Caltha L.}

142. Caltha palustris L. (syn.: Caltha polypetala Hochst. ex Lorent) - Lilpar, Bataklık nergisi

Ormanüstü plateau, streamside, $2000 \mathrm{~m}$, 10.05.2005, Uzun 561 (KATO: 17545).

\section{Aconitum L.}

143. Aconitum orientale Mill.

Ormanüstü village, in forest, $1707 \mathrm{~m}$, 16.07.2008, Uzun 1573 (KATO: 17546); Ormanüstü village, damp area in forest, 1620 m, 16.07.2008, Uzun 1574 (KATO: 17547); Ormanüstü village, in forest, $1600 \mathrm{~m}$, 02.08.2008, Uzun 1759 (KATO: 17548). Eux. el.

\section{Anemone L. - Kır lalesi}

144. Anemone blanda Schott \& Kotschy Ormanüstü village, shrubby place, $1150 \mathrm{~m}$, 10.04.2005, Uzun 551 (KATO: 17549).

145. Anemone caucasica Willd ex Rupr.Kafkas kır lalesi

Ormanüstü plateau, grassy place, $1850 \mathrm{~m}$, 10.04.2005, Uzun 552 (KATO: 17550). Eux. (mt.) el., Rare, IUCN: VU,

\section{Clematis $\mathbf{L}$.}

146. Clematis vitalba L. - Orman Asmas 1 Ormanüstü village, shrubby place, $1300 \mathrm{~m}$, 08.07.2005, Uzun 621 (KATO: 17551); Köprüyanı district, shrubby place, $600 \mathrm{~m}$, 23.05.2006, Uzun 861 (KATO: 17552); Sukenar1 district, shrubby place, $685 \mathrm{~m}$, 11.07.2007, Uzun 969 (KATO: 17553).

\section{Ranunculus L. - Düğün çiçeği}

147. Ranunculus cappadocicus Willd.

Köprüyanı district, damp places in forest, 1130 m, 23.05.2006, Uzun 862 (KATO: 17554). Eux. el., Relict

148. Ranunculus brutius Ten.

Ormanüstü village, in forest, $1948 \mathrm{~m}$, 22.07.2008, Uzun 1707 (KATO: 17555). Euro-Sib. el.

149. Ranunculus repens $\mathrm{L}$.

Ormanüstü village, damp place, $1600 \mathrm{~m}$, 02.08.2008, Uzun 1760 (KATO: 17556). 
150. Ranunculus bulbosus L. subsp. aleae (Willk.) Rouy \& Foucaud

Ormanüstü village, damp place, $1600 \mathrm{~m}$, 02.08.2008, Uzun 1761 (KATO: 17557).

Euro-Sib. el., Rare, IUCN: VU

151. Ranunculus constantinopolitanus (DC.) d'Urv. - İstanbul düğün çiçeği

Ormanüstü village, grassy place, $1100 \mathrm{~m}$, 14.07.2008, Uzun 1455 (KATO: 17558).

\section{Aquilegia L. - Haseki küpesi}

152. Aquilegia olympica Boiss.

Ormanüstü village, grassy place, $1000 \mathrm{~m}$, 10.05.2005, Uzun 562 (KATO: 17559).

Proteales Juss. ex Bercht. \& J.Presl

F32 [4]. Platanaceae T.Lestib.

\section{Platanus L. - Çınar}

153. Platanus orientalis L. - Doğu çınarı

Çeşmeler district, streamside, $460 \mathrm{~m}$, 01.06.2008, Uzun 1052 (KATO: 17566).

\section{CORE EUDICOTS \\ SUPERROSIDS}

Saxifragales Bercht. \& J.Presl

F33 [5]. Grossulariaceae DC.

\section{Ribes L. - Frenk üzümü}

154. Ribes petraeum Wulfen (syn.: Ribes biebersteinii Berl. ex DC.)

Çamlıdüz plateau, in forest, $1668 \mathrm{~m}$, 17.07.2008, Uzun 1598 (KATO: 17756); Ormanüstü village, forest edge, $1445 \mathrm{~m}$, 17.07.2008, Uzun 1599 (KATO: 17757). Eux. el.

155. Ribes alpinum $\mathrm{L}$.

Ormanüstü village, mixed forest with Fagus orientalis-Picea orientalis, $1606 \mathrm{~m}$, 10.07.2008, Uzun 1371 (KATO: 17758); Ormanüstü village, open forest, $1627 \mathrm{~m}$, 15.07.2008, Uzun 1550 (KATO: 17759); ibid., 1639 m, 15.07.2008, Uzun 1551 (KATO: 17760); Ormanüstü village, in forest, $1691 \mathrm{~m}, 16.07 .2008$, Uzun 1579 (KATO: 17761); Ormanüstü village, in forest, $1475 \mathrm{~m}, 21.07 .2008$, Uzun 1685 (KATO: 17762); ibid., 1745 m, 21.07.2008, Uzun 1686 (KATO: 17763); Ormanüstü village, in forest, $1205 \mathrm{~m}, 24.07 .2008$, Uzun 1735 (KATO: 17764). Euro-Sib. el.

\section{F34 [6]. Saxifragaceae Juss.}

\section{Saxifraga L. - Taşkıran otu}

156. Saxifraga paniculata Mill.

Ormanüstü village, rock cracks, $1680 \mathrm{~m}$, 08.07.2005, Uzun 650 (KATO: 17789); ibid., $1577 \mathrm{~m}$, 08.07.2005, Uzun 651 (KATO: 17790); Ormanüstü village, rocky place, $1577 \mathrm{~m}, 05.08 .2005$, Uzun 774 (KATO: 17791); Ormanüstü plateau, rock cracks, 2000 m, 05.06.2006, Uzun 904 (KATO: 17792). Euro-Sib. el.

157. Saxifraga rotundifolia L. subsp. rotundifolia

Ormanüstü village, wet rocky hills, $1300 \mathrm{~m}$, 08.07.2005, Uzun 652 (KATO: 17793); Ormanüstü village, forest edge, rocky hills, $1600 \mathrm{~m}, 05.08 .2005$, Uzun 775 (KATO: 17794). Euro-Sib. el.

158. Saxifraga cymbalaria L. (syn.: Saxifraga cymbalaria L. var. huetiana (Boiss.) Engler \& Irmscher)

Ormanüstü village, wet rocky place, 1500 $1750 \mathrm{~m}, 08.07 .2005$, Uzun 653 (KATO: 17796). Köprüyan1, over wet rock, $930 \mathrm{~m}$, 23.05.2006, Uzun 870 (KATO: 17795).

\section{F35 [7]. Crassulaceae J.St.-Hil. \\ 95. Phedimus Raf.}

159. Phedimus spurius (M.Bieb.) 't Hart (syn.: Sedum spurium M.Bieb.)

Ormanüstü plateau, stony place, $1945 \mathrm{~m}$, 08.07.2005, Uzun 645 (KATO: 17765); Ormanüstü village, stony hill, $1100 \mathrm{~m}$, 05.08.2005, Uzun 773 (KATO: 17766); ibid., 850 m, 26.07.2006, Uzun 943 (KATO: 17767). Hyr.-Eux. el.

160. Phedimus stoloniferus (S.G.Gmel.) ' $\mathrm{t}$ Hart (syn.: Sedum stoloniferum S.G.Gmel.)

Köprüyan1 district, in forest, $1001 \mathrm{~m}$, 08.07.2008, Uzun 1284 (KATO: 17768); Ormanüstü village, in forest, $1280 \mathrm{~m}$, 09.07.2008, Uzun 1341 (KATO: 17769). Hyr.-Eux. (mt.) el.

\section{Sedum L. - Dam koruğu}

161. Sedum acre L.

Çeşmeler district, stony hill, $1050 \mathrm{~m}$, 11.07.2007, Uzun 976 (KATO: 17770.

162. Sedum gracile C.A.Mey.

Ormanüstü plateau, stony place, $1945 \mathrm{~m}$, 08.07.2005, Uzun 646 (KATO: 17771); Ormanüstü village, coniferous-deciduous 
mixed forest, $1172 \mathrm{~m}$, 10.07.2008, Uzun 1372 (KATO: 17772); Ormanüstü village, in forest, $1467 \mathrm{~m}$, 12.07.2008, Uzun 1447 (KATO: 17773); Çamlıdüz plateau, afforestation site, $1728 \mathrm{~m}, 20.07 .2008$, Uzun 1643 (KATO: 17774). Hyr.-Eux. (mt.) el.

163. Sedum hispanicum L.

Ormanüstü village, stony hill, $1750 \mathrm{~m}$, 08.07.2005, Uzun 647 (KATO: 17775); ibid., 1615 m, 26.07.2006, Uzun 648 (KATO: 17776).

164. Sedum pallidum M.Bieb. var. pallidum Ormanüstü plateau, grassy place, $2000 \mathrm{~m}$, 05.06.2006, Uzun 903 (KATO: 17777); Çeşmeler district, roadside, $910 \mathrm{~m}$, 18.06.2008, Uzun 1095 (KATO: 17778); Çeşmeler district, stony hill, $634 \mathrm{~m}$, 07.07.2008, Uzun 1143 (KATO: 17779); Köprüyanı district, open forest, $701 \mathrm{~m}$, 08.07.2008, Uzun 1285 (KATO: 17780); Çamlıdüz plateau, afforestation site, $1728 \mathrm{~m}$, 20.07.2008, Uzun 1644 (KATO: 17781).

165. Sedum pallidum M.Bieb. var. bithynicum (Boiss.) Chamb.

Ormanüstü plateau, stony place, $1945 \mathrm{~m}$, 08.07.2005, Uzun 649 (KATO: 17782); Çeşmeler district, stony hill, $1050 \mathrm{~m}$, 11.07.2007, Uzun 977 (KATO: 17783); Çeşmeler district, stony hill, $437 \mathrm{~m}$, 07.07.2008, Uzun 1144 (KATO: 17784); Köprüyanı district, rocky place, $1017 \mathrm{~m}$, 08.07.2008, Uzun 1286 (KATO: 17785); Ormanüstü village, afforestation site, 1683 m, 10.07.2008, Uzun 1373 (KATO: 17786); Sukenar1 district, open forest, $498 \mathrm{~m}$, 11.07.2008, Uzun 1413 (KATO: 17787). Eux. el.

\section{Sempervivum L. - Gelin parmağı}

166. Sempervivum armenum Boiss. \& A.Huet var. armenum

Köprüyan1, roadside slope, $750 \mathrm{~m}$, 11.07.2007, Uzun 978 (KATO: 17788). Eux. (mt.) el., Endemic, IUCN: NT

\section{ROSIDS}

Vitales Juss. ex Bercht. \& J.Presl

F36 [8]. Vitaceae Juss.

98. Vitis L.

167. Vitis vinifera L. (syn.: Vitis sylvestris C.C.Gmel.) - Yabani üzüm
Çatak, rocky slopes, $400 \mathrm{~m}, 11.07 .2008$, Uzun 1422 (KATO: 18005).

Fabales Bromhead

F37 [9]. Fabaceae Lindl. (= Leguminosae Juss.)

99. Genista L.

168. Genista tinctoria L. - Katır tırnağı

Çeşmeler district, stony rocky hill, $750 \mathrm{~m}$, 01.06.2008, Uzun 1060 (KATO: 17869); Çeşmeler district, stony hill, $437 \mathrm{~m}$, 07.07.2008, Uzun 1153 (KATO: 17870); Çeşmeler district, stony hills, $490 \mathrm{~m}$, 07.07.2008, Uzun 1154 (KATO: 17871). Euro-Sib. el.

100. Argyrolobium Eckl. \& Zeyh.

169. Argyrolobium biebersteinii P.W.Ball

Köprüyan1 district, rocky place, $860 \mathrm{~m}$, 08.07.2008, Uzun 1293 (KATO: 17872); Köprüyanı district, in forest, $964 \mathrm{~m}$, 08.07.2008, Uzun 1294 (KATO: 17873); Çatak, open forest, $458 \mathrm{~m}, 14.07 .2008$, Uzun 1461 (KATO: 17874); Çatak, afforestation site, 477 m, 14.07.2008, Uzun 1462 (KATO: 17875); Catak, hill, 1000 m, 14.07.2008, Uzun 1463 (KATO: 17876); Çatak, afforestation site, $873 \mathrm{~m}, 06.08 .2008$, Uzun 1821 (KATO: 17877).

\section{Robinia L.}

170. Robinia pseudoacacia L. - Beyaz çiçekli yalancı akasya

Köprüyan1 district, roadside, $700 \mathrm{~m}$, 23.05.2006, Uzun 875 (KATO: 17878). Exotic

\section{Astragalus L. - Geven}

171. Astragalus glycyphyllos L.

Ormanüstü village, roadside slope, $929 \mathrm{~m}$, 08.07.2005, Uzun 666 (KATO: 17879). Eux. el.

172. Astragalus ovatus DC.

Çeşmeler district, grassy place, $700 \mathrm{~m}$, 01.06.2008, Uzun 1061 (KATO: 17880); ibid., 910 m, 18.06.2008, Uzun 1097 (KATO: 17881); Çeşmeler district, stony hill, $583 \mathrm{~m}$, 07.07.2008, Uzun 1155 (KATO: 17882). Endemic, IUCN: previously DD, but revised as EN by Ekici (2000) 
173. Astragalus viciifolius DC.

Çeşmeler district, stony hill, $490 \mathrm{~m}$, 07.07.2008, Uzun 1156 (KATO: 17883); Çatak, afforestation site, $873 \mathrm{~m}, 06.08 .2008$, Uzun 1822 (KATO: 17884).

174. Astragalus viridissimus Freyn \& Sint.

Çeşmeler district, stony hill, $583 \mathrm{~m}$, 07.07.2008, Uzun 1157 (KATO: 17885); Yukarıköy village, stony hill, $800 \mathrm{~m}$, 14.07.2008, Uzun 1464 (KATO: 17886). Eux. el., Endemic, IUCN: LC

175. Astragalus ansinii Uzun, Terzioğlu \& Pal.-Uzun

Çeșmeler district, stony hill, $750 \mathrm{~m}$, 18.06.2008, Uzun 492 (KATO: 17887); Çeşmeler district, stony hill, $632 \mathrm{~m}$, 11.07.2008, Uzun 493 (KATO: 17888). Eux. el., Endemic, IUCN: CR

\section{Bituminaria C.H.Stirt.}

176. Bituminaria bituminosa (L.) C.H.Stirt. (syn.: Psoralea bituminosa L.)

Çeşmeler district, open place, $440 \mathrm{~m}$, 08.07.2005, Uzun 667 (KATO: 17889; Köprüyan1, forest edge, 900 m, 11.07.2007, Uzun 982 (KATO: 17890); Çeşmeler district, stony hills, $490 \mathrm{~m}$, 07.07.2008, Uzun 1158 (KATO: 17891); Sukenar1 district, forest edge, $663 \mathrm{~m}, 07.07 .2008$, Uzun 1159 (KATO: 17892); Çatak, afforestation site, $477 \mathrm{~m}, 14.07 .2008$, Uzun 1465 (KATO: 17893). Medit. el.

\section{Vicia L. - Fiğ}

177. Vicia cracca L. subsp. cracca

Ormanüstü village, grassy place, $1000 \mathrm{~m}$, 08.07.2005, Uzun 668 (KATO: 17894). Euro-Sib. el.

178. Vicia villosa Roth

Çeşmeler district, roadside, $650 \mathrm{~m}$, 01.06.2008, Uzun 1062 (KATO: 17895); Yukarıköy village, afforestation site, $836 \mathrm{~m}$, 18.07.2008, Uzun 1628 (KATO: 17896); Çamlıdüz plateau, in forest, $1769 \mathrm{~m}$, 22.07.2008, Uzun 1714 (KATO: 17897).

179. Vicia sepium $\mathrm{L}$.

Ormanüstü village, afforestation site, 1683 m, 10.07.2008, Uzun 1376 (KATO: 17898).

Euro-Sib. el.

180. Vicia sativa $\mathrm{L}$.

Ormanüstü village, open place, $1500 \mathrm{~m}$, 05.08.2005, Uzun 778 (KATO: 17899). Cosmopolitan
*181. Vicia segetalis Thuill (syn.: Vicia sativa L. subsp. nigra (L.) Ehrh. var. segetalis (Thuill.) Ser. ex DC.)

Ormanüstü village, grassy place, $980 \mathrm{~m}$, 08.07.2005, Uzun 669 (KATO: 17900).

\section{Lathyrus L. - Mürdümük}

182. Lathyrus aureus (G.Lodd. ex Drapiez) D.Brândză

Ormanüstü village, in forest, $1745 \mathrm{~m}$, 21.07.2008, Uzun 1689 (KATO: 17901). Eux. el.

183. Lathyrus vernus (L.) Bernh.

Köprüyan1 district, in forest, $964 \mathrm{~m}$, 08.07.2008, Uzun 1295 (KATO: 17902); Çatak, in forest, $486 \mathrm{~m}, 14.07 .2008$, Uzun 1466 (KATO: 17903); Ormanüstü village, in forest, $1205 \mathrm{~m}, 24.07 .2008$, Uzun 1738 (KATO: 17904); Çeşmeler district, degraded spruce forest, $541 \mathrm{~m}, 06.08 .2008$, Uzun 1823 (KATO: 17905). Euro-Sib. el.

184. Lathyrus laxiflorus (Desf.) O.Kuntze subsp. laxiflorus

Köprüyanı district, open forest, $600 \mathrm{~m}$, 23.05.2006, Uzun 876 (KATO: 17906); Çatak, in forest, $486 \mathrm{~m}, 14.07 .2008$, Uzun 1467 (KATO: 17907).

\section{Ononis L.}

*185. Ononis pusilla L.

Çeşmeler district, stony hill, $490 \mathrm{~m}$, 07.07.2008, Uzun 1160 (KATO: 17908). Medit. el.

\section{Trifolium L. - Üçgül}

186. Trifolium repens $\mathrm{L}$.

Ormanüstü village, grassy place, $950 \mathrm{~m}$, 08.07.2005, Uzun 670 (KATO: 17909); Ormanüstü village, open forest, $1770 \mathrm{~m}$, 08.07.2005, Uzun 671 (KATO: 17910).

187. Trifolium spadiceum $\mathrm{L}$.

Ormanüstü village, streamside, $1600 \mathrm{~m}$, 17.07.2008, Uzun 1601 (KATO: 17911). Euro-Sib. el.

188. Trifolium aureum Pollich subsp. aureum Çatak, afforestation site, $873 \mathrm{~m}, 06.08 .2008$, Uzun 1824 (KATO: 17912). Euro-Sib. el. 189. Trifolium pratense $\mathrm{L}$. subsp. pratense Ormanüstü plateau, afforestation site, 1862 m, 02.08.2008, Uzun 1771 (KATO: 17913); Ormanüstü plateau, degraded spruce forest, 1991 m, 02.08.2008, Uzun 1772 (KATO: 17914), Cosmopolitan 
190. Trifolium ochroleucon Huds.

Ormanüstü plateau, grassy place, $2000 \mathrm{~m}$, 05.06.2006, Uzun 908 (KATO: 17915); Sukenar1 district, stony hill, $663 \mathrm{~m}$, 07.07.2008, Uzun 1161 (KATO: 17916); Köprüyanı district, in forest, $701 \mathrm{~m}$, 08.07.2008, Uzun 1296 (KATO: 17917); Köprüyanı district, rocky place, $1017 \mathrm{~m}$, 08.07.2008, Uzun 1297 (KATO: 17918); Çatak, open forest, $458 \mathrm{~m}, 14.07 .2008$, Uzun 1468 (KATO: 17919); Çatak, afforestation site, 477 m, 14.07.2008, Uzun 1469 (KATO: 17920.

191. Trifolium canescens Willd.

Ormanüstü village, grassy place, $1680 \mathrm{~m}$, 08.07.2005, Uzun 672 (KATO: 17921); Ormanüstü village, afforestation site, 1683 m, 10.07.2008, Uzun 1377 (KATO: 17922). Hyr.-Eux. el.

192. Trifolium arvense L. subsp. arvense

Köprüyanı district, in forest, $701 \mathrm{~m}$, 08.07.2008, Uzun 1298 (KATO: 17923); Köprüyanı district, rocky place, $1017 \mathrm{~m}$, 08.07.2008, Uzun 1299 (KATO: 17924).

\section{Trigonella L. - Taş yoncası}

193. Trigonella officinalis (L.) Coulot \& Rabaute (syn.: Melilotus officinalis (L.) Desr.)

Ormanüstü village, roadside, $900 \mathrm{~m}$, 05.08.2005, Uzun 779 (KATO: 17925); Çeşmeler district, roadside, $700 \mathrm{~m}$, 26.07.2006, Uzun 945 (KATO: 17926); Sukenar1 district, roadside, $685 \mathrm{~m}$, 11.07.2007, Uzun 983 (KATO: 17927).

\section{Medicago L. - Yonca}

*194. Medicago orbicularis (L.) Bartal. Çeşmeler district, stony hill, $632 \mathrm{~m}$, 11.07.2008, Uzun 1416 (KATO: 17928).

195. Medicago lupulina $\mathrm{L}$.

Ormanüstü village, open place, $440 \mathrm{~m}$, 08.07.2005, Uzun 673 (KATO: 17929); Çatak, afforestation site, $477 \mathrm{~m}, 14.07 .2008$, Uzun 1470 (KATO: 17930).

196. Medicago sativa $\mathrm{L}$.

Sukenar1 village, roadside, $420 \mathrm{~m}$, 11.07.2008, Uzun 1417 (KATO: 17931).

197. Medicago $\times$ varia Martyn

Çeşmeler district, stony hill, $634 \mathrm{~m}$, 07.07.2008, Uzun 1162 (KATO: 17932); ibid., 437 m, 07.07.2008, Uzun 1163 (KATO: 17933).
198. Medicago falcata $\mathrm{L}$.

Çeşmeler district, stony hill, $1050 \mathrm{~m}$, 11.07.2007, Uzun 948 (KATO: 17934); Çeşmeler district, roadside slope, $720 \mathrm{~m}$, 01.06.2008, Uzun 1063 (KATO: 17935); Çeşmeler district, stony-grassy place, $890 \mathrm{~m}$, 18.06.2008, Uzun 1099 (KATO: 17936); Çeşmeler district, stony hill, $490 \mathrm{~m}$, 07.07.2008, Uzun 1164 (KATO: 17937); Çeşmeler district, stony hill, $632 \mathrm{~m}$, 11.07.2008, Uzun 1418 (KATO: 17938); Çatak, open forest, $458 \mathrm{~m}, 14.07 .2008$, Uzun 1471 (KATO: 17939); Çatak, roadside slope, $1000 \mathrm{~m}, 14.07 .2008$, Uzun 1472 (KATO: 17940).

199. Medicago minima (L.) L.

Çeşmeler district, stony hill, $632 \mathrm{~m}$, 11.07.2008, Uzun 1419 (KATO: 17941).

\section{Lotus L. - Gazelboynuzu}

200. Lotus graecus L. (syn.: Dorycnium graecum (L.) Ser.)

Köprüyan1, roadside slope, $730 \mathrm{~m}$, 11.07.2007, Uzun 985 (KATO: 17942). Eux. el.

201. Lotus herbaceus (Vill.) Jauzein (syn.: Dorycnium pentaphyllum Scop. subsp. herbaceum (Vill.) Rouy)

Çeşmeler district, roadside, $444 \mathrm{~m}$, 08.07.2005, Uzun 674 (KATO: 17943); ibid., open place, $458 \mathrm{~m}, 05.08 .2005$, Uzun 780 (KATO: 17944); Köprüyanı, roadside slope, 700 m, 11.07.2007, Uzun 986 (KATO: 17945); Çeşmeler district, stony hill, $583 \mathrm{~m}$, 07.07.2008, Uzun 1165 (KATO: 17946); ibid., 634 m, 07.07.2008, Uzun 1166 (KATO: 17947); Sukenarı district, open forest, $498 \mathrm{~m}$, 11.07.2008, Uzun 1420 (KATO: 17948); Çatak, open forest, $458 \mathrm{~m}, 14.07 .2008$, Uzun 1473 (KATO: 17949); ibid. in forest, $483 \mathrm{~m}$, 14.07.2008, Uzun 1474 (KATO: 17950).

202. Lotus corniculatus L. subsp. corniculatus

Çatak, in forest, $1010 \mathrm{~m}, 14.07 .2008$, Uzun 1475 (KATO: 17951); Ormanüstü village, roadside slope, $1230 \mathrm{~m}, 13.08 .2005$, Uzun 855 (KATO: 17952).

203. Lotus corniculatus L. subsp. alpinus (DC.) Rothm.

Ormanüstü plateau, grassy place, $2000 \mathrm{~m}$, 05.06.2006, Uzun 909 (KATO: 17953); Ormanüstü village, afforestation site, 1683 m, 10.07.2008, Uzun 1378 (KATO: 17954); 
Ormanüstü plateau, degraded spruce forest, 1991 m, 02.08.2008, Uzun 1773 (KATO: 17955).

\section{Anthyllis L.}

204. Anthyllis vulneraria L. subsp. pulchella (Vis.) Bornm.

Ormanüstü plateau, grassy place, $2000 \mathrm{~m}$, 16.07.2008, Uzun 1580 (KATO: 17956).

\section{Securigera DC.}

205. Securigera varia (L.) Lassen (syn.: Coronilla varia L. subsp. varia) - Körigen Ormanüstü village, open place, $1700 \mathrm{~m}$, 08.07.2005, Uzun 675 (KATO: 17957); Çeşmeler district, stony hills, $490 \mathrm{~m}$, 07.07.2008, Uzun 1167 (KATO: 17958); Çatak, afforestation site, 477 m, 14.07.2008, Uzun 1476 (KATO: 17959).

206. Securigera orientalis (Mill.) Lassen subsp. balansae (Boiss.) Keskin (syn.: Coronilla orientalis Mill. var. balansae (Boiss.) Uhrova)

Çeşmeler district, roadside slope, $850 \mathrm{~m}$, 11.07.2007, Uzun 987 (KATO: 17960).

\section{Scorpiurus L.}

207. Scorpiurus subvillosus L. (syn.: Scorpiurus muricatus L. var. subvillosus (L.) Fiori)

Çeşmeler district, roadside slope, $700 \mathrm{~m}$, 05.06.2006, Uzun 910 (KATO: 17961. Medit. el.

\section{Onobrychis Adans.}

208. Onobrychis arenaria (Kit.) DC. subsp. cana (Boiss.) Hayek (syn.: Onobrychis oxyodonta Boiss. var. armena (Boiss. \& A. Huet) Ponert, Onobrychis armena Boiss. \& A. Huet)

Çeşmeler district, shrubby place, $800 \mathrm{~m}$, 01.06.2008, Uzun 1064 (KATO: 17962); Çeşmeler district, stony hills, $490 \mathrm{~m}$, 07.07.2008, Uzun 1168 (KATO: 17963); ibid., 583 m, 07.07.2008, Uzun 1169 (KATO: 17964). Endemic, IUCN: LC

\section{F38 [10]. Polygalaceae Hoffmanns. \& Link}

115. Polygala L.

209. Polygala pruinosa Boiss. subsp. pruinosa
Catak, in forest, $483 \mathrm{~m}, 14.07 .2008$, Uzun 1479 (KATO: 18014); Çeşmeler district, degraded spruce forest, $541 \mathrm{~m}, 06.08 .2008$, Uzun 1827 (KATO: 18015).

210. Polygala major Jacq.

Ormanüstü plateau, open place, $1800 \mathrm{~m}$, 05.08.2005, Uzun 784 (KATO: 18016); Çeşmeler district, in Carpinus orientalis forest, $890 \mathrm{~m}$, 18.06.2008, Uzun 1101 (KATO: 18017). Euro-Sib. el.

211. Polygala comosa Schkuhr

Ormanüstü plateau, grassy place, $1815 \mathrm{~m}$, 08.07.2005, Uzun 680 (KATO: 18018).

212. Polygala alpestris Rchb. subsp. alpestris

Ormanüstü plateau, grassy place, $2000 \mathrm{~m}$, 05.06.2006, Uzun 914 (KATO: 18019); ibid., $1850 \mathrm{~m}, 16.07 .2008$, Uzun 1581 (KATO: 18020); Ormanüstü plateau, degraded spruce forest, $1991 \mathrm{~m}$, 02.08.2008, Uzun 1775 (KATO: 18021). Euro-Sib. el.

Rosales Bercht. \& J.Presl

F39 [11]. Rosaceae Juss.

\section{Aruncus Adanson}

213. Aruncus dioicus (Walter) Fernald (syn.: Aruncus vulgaris L.) -Kamçıbıyık

Ormanüstü village, deciduous forest edge, $1300 \mathrm{~m}, 10.05 .2005$, Uzun 575 (KATO: 17797).

\section{Prunus L.}

214. Prunus laurocerasus L. (syn.: Laurocerasus officinalis M.Roem.) Karayemiş

Ormanüstü village, damp creekside, $1560 \mathrm{~m}$, 05.06.2006, Uzun 905 (KATO: 17798).

215. Prunus domestica L. - Erik Ormanüstü village, roadside, $1100 \mathrm{~m}$, 05.06.2006, Uzun 906 (KATO: 17799).

216. Prunus ceracifera Ehrh. (syn.: Prunus divaricata Ledeb. var. divaricate)

Kiremitli district, roadside, $900 \mathrm{~m}$, 13.07.2007, Uzun 1034 (KATO: 17800). 217. Prunus avium (L.) L. (syn.: Cerasus avium (L.) Moench) - Kiraz

Ormanüstü village, roadside, $1370 \mathrm{~m}$, 10.05.2005, Uzun 576 (KATO: 17801). 
118. Rubus L. - Böğürtlen

218. Rubus idaeus L. subsp. idaeus Ahududu

Çamlıdüz plateau, forest edge, $1769 \mathrm{~m}$, 22.07.2008, Uzun 1711 (KATO: 17802).

219. Rubus sanctus Schreb.

Ormanüstü village, roadside, $1200 \mathrm{~m}$, 08.07.2005, Uzun 654 (KATO: 17803);

Köprüyanı district, forest edge, $600 \mathrm{~m}$, 23.05.2006, Uzun 871 (KATO: 17804).

220. Rubus hirtus Waldst. \& Kit. group.

Köprüyanı district, forest edge, $910 \mathrm{~m}$, 23.05.2006, Uzun 872 (KATO: 17805). Eux. el.

221. Rubus caucasicus Focke

Ormanüstü village, in forest, $1600 \mathrm{~m}$, 05.06.2006, Uzun 907 (KATO: 17806). Eux. el.

\section{Potentilla L. - Beşparmak otu}

222. Potentilla argentea $\mathrm{L}$.

Çeşmeler district, grassy place, $800 \mathrm{~m}$, 18.06.2008, Uzun 1096 (KATO: 17807).

223. Potentilla erecta (L.) Raeusch.

Ormanüstü plateau, grassy place, $1945 \mathrm{~m}$, 08.07.2005, Uzun 655 (KATO: 17808); Çamlıdüz plateau, afforestation site, $1728 \mathrm{~m}$, 20.07.2008, Uzun 1645 (KATO: 17809).

224. Potentilla elatior Willd. ex D.F.K.Schltdl.

Ormanüstü village, grassy place, $1162 \mathrm{~m}$, 09.07.2008, Uzun 1342 (KATO: 17810); Çamlıdüz plateau, in forest, $1769 \mathrm{~m}$, 22.07.2008, Uzun 1712 (KATO: 17811). Eux. el.

225. Potentilla micrantha Ramond ex DC. Ormanüstü plateau, afforestation site, 1664 m, 21.07.2008, Uzun 1688 (KATO: 17812).

\section{Fragaria $\mathbf{L}$.}

226. Fragaria vesca L. - Orman çileği Köprüyan1, roadside slope, $930 \mathrm{~m}$, 23.05.2006, Uzun 873 (KATO: 17813).

\section{Sibbaldia L.}

227. Sibbaldia parviflora Willd. var. parviflora

Ormanüstü plateau, grassy place, $1800 \mathrm{~m}$, 10.05.2005, Uzun 577 (KATO: 17814).

122. Geum L.

228. Geum coccineum $\mathrm{Sm}$.
Ormanüstü plateau, grassy place, $1900 \mathrm{~m}$, 05.08.2005, Uzun 776 (KATO: 17815). Euro-Sib. el.

229. Geum urbanum L. - Su karanfili

Köprüyanı district, grassy place, $600 \mathrm{~m}$, 23.05.2006, Uzun 874 (KATO: 17816). Euro-Sib. el.

\section{Agrimonia L.}

230. Agrimonia eupatoria L. subsp. eupatoria

Çeşmeler district, forest edge, $595 \mathrm{~m}$, 08.07.2005, Uzun 656 (KATO: 17817); Ormanüstü village, roadside slope, $700 \mathrm{~m}$, 26.07.2006, Uzun 944 (KATO: 17818); Köprüyan1, forest edge, 900 m, 11.07.2007, Uzun 979 (KATO: 17819).

\section{Aremonia Nestler}

231. Aremonia agrimonoides (L.) DC. subsp. agrimonoides

Ormanüstü plateau, pure Picea orientalis stand, $1614 \mathrm{~m}, 10.07 .2008$, Uzun 1374 (KATO: 17820); Ormanüstü village, in forest, $1479 \mathrm{~m}$, 12.07.2008, Uzun 1449 (KATO: 17821). Euro-Sib. el.

\section{Poterium L.}

232. Poterium sanguisorba L. subsp. balearicum (Bourg. ex Nyman) Stace (syn.: Sanguisorba minor Scop. subsp. muricata (Spach) Briq.)

Ormanüstü village, open hilly place, $929 \mathrm{~m}$, 08.07.2005, Uzun 657 (KATO: 17822); Çeşmeler district, stony hill, $1050 \mathrm{~m}$, 11.07.2007, Uzun 980 (KATO: 17823).

\section{Alchemilla L. - Aslan pençesi}

233. Alchemilla caucasica Buser

Ormanüstü plateau, grassy place, $1900 \mathrm{~m}$, 08.07.2005, Uzun 658 (KATO: 17824); Çamlıdüz plateau, afforestation site, $1728 \mathrm{~m}$, 20.07.2008, Uzun 1646 (KATO: 17825); Ormanüstü plateau, afforestation site, 1862 m, 02.08.2008, Uzun 1766 (KATO: 17826); Ormanüstü plateau, degraded spruce forest, $1991 \mathrm{~m}, 02.08 .2008$, Uzun 1767 (KATO: 17827). Eux. (mt.) el.

*234. Alchemilla valdehirsuta Buser

Ormanüstü village, roadside damp place, $1550 \mathrm{~m}, 08.07 .2005$, Uzun 659 (KATO: 17828); Ormanüstü village, grassy place, 1162 m, 09.07.2008, Uzun 1343 (KATO: 
17829); Çamlidüz plateau, afforestation site, 1728 m, 20.07.2008, Uzun 1647 (KATO: 17830).

235. Alchemilla orthotricha Rothm.

Ormanüstü village, roadside damp place, 1550 m, 08.07.2005, Uzun 660 (KATO: 17831). Eux. (mt.) el., Rare, IUCN: VU

236. Alchemilla holotricha Juz.

Ormanüstü village, grassy place, $1162 \mathrm{~m}$, 09.07.2008, Uzun 1344 (KATO: 17832).

Rare, IUCN: VU

237. Alchemilla hirtipedicellata Juz.

Ormanüstü plateau, afforestation site, 1862 m, 02.08.2008, Uzun 1768 (KATO: 17833); Ormanüstü plateau, stony degraded spruce forest, $2010 \mathrm{~m}$, 02.08.2008, Uzun 1769 (KATO: 17834). Eux. (mt.) el.

238. Alchemilla mollis (Buser) Rothm

Ormanüstü village, damp hill, 1500-1750 m, 08.07.2005, Uzun 661 (KATO: 17835); Çamlıdüz plateau, damp hill, $1574 \mathrm{~m}$, 17.07.2008, Uzun 1600 (KATO: 17836); Çamlıdüz plateau, afforestation site, $1728 \mathrm{~m}$, 20.07.2008, Uzun 1648 (KATO: 17837).

239. Alchemilla speciosa Buser

Ormanüstü village, forest edge, $1750 \mathrm{~m}$, 10.05.2005, Uzun 578 (KATO: 17838); Ormanüstü village, damp hill, 1500-1750 m, 08.07.2005, Uzun 662 (KATO: 17839); Çamlıdüz plateau, afforestation site, $1728 \mathrm{~m}$, 20.07.2008, Uzun 1649 (KATO: 17840); Ormanüstü plateau, degraded spruce forest, 1991 m, 02.08.2008, Uzun 1770 (KATO: 17841). Rare, IUCN: VU

240. Alchemilla persica Rothm.

Ormanüstü village, roadside damp place, $1550 \mathrm{~m}$, 08.07.2005, Uzun 663 (KATO: 17842). Ir.-Tur. el.

*241. Alchemilla ikizdereensis Kalheber Ormanüstü village, roadside damp place, $1550 \mathrm{~m}$, 08.07.2005, Uzun 664 (KATO: 17843). Eux. el., Endemic, IUCN: EN

\section{Rosa L. - Yabani gül, Kuşburnu}

242. Rosa boissieri Crép. (syn.: Rosa montana Chaix subsp. woronowii (Lonacz.) Ö.Nilsson)

Çamlidüz plateau, in forest, $1769 \mathrm{~m}$, 22.07.2008, Uzun 1713 (KATO: 17844).

243. Rosa canina L. - Yabani gül

Ormanüstü village, open forest, $1770 \mathrm{~m}$, 08.07.2005, Uzun 665 (KATO: 17845); Sukenar1 district, forest edge, $640 \mathrm{~m}$,
07.07.2008, Uzun 1145 (KATO: 17846); Çeşmeler district, stony hills, $490 \mathrm{~m}$, 07.07.2008, Uzun 1146 (KATO: 17847); Köprüyanı district, rocky place, $860 \mathrm{~m}$, 08.07.2008, Uzun 1287 (KATO: 17848).

\section{Cotoneaster Medik. - Taş elması}

244. Cotoneaster morulus Pojark

Çeşmeler district, stony hill, $490 \mathrm{~m}$, 07.07.2008, Uzun 1147 (KATO: 17849).

\section{Pyracantha M.Roem.}

245. Pyracantha coccinea M.Roem. - Ateş dikeni, Tavşan elması

Çeşmeler district, çalılık, 583 m, 07.07.2008, Uzun 1148 (KATO: 17850); Çeşmeler district, stony hills, $634 \mathrm{~m}, 07.07 .2008$, Uzun 1149 (KATO: 17851).

\section{Crataegus L. - Alıç}

246. Crataegus rhipidophylla Gand. var. rhipidophylla (syn.: Crataegus curvisepala Lindman)

Sukenar1 district, in forest, $663 \mathrm{~m}$, 07.07.2008, Uzun 1150 (KATO: 17852); Köprüyanı district, in forest, $964 \mathrm{~m}$, 08.07.2008, Uzun 1288 (KATO: 17853); Çatak, in forest, $1010 \mathrm{~m}, 14.07 .2008$, Uzun 1459 (KATO: 17854); Çatak, in forest, 486 m, 14.07.2008, Uzun 1460 (KATO: 17855); Çeşmeler district, degraded spruce forest, $541 \mathrm{~m}, 06.08 .2008$, Uzun 1818 (KATO: 17856).

247. Crataegus monogyna Jacq. var. monogyna-Geyik dikeni

Çeșmeler district, in forest, $1020 \mathrm{~m}$, 08.07.2008, Uzun 1289 (KATO: 17857); Çatak, afforestation site, $873 \mathrm{~m}, 06.08 .2008$, Uzun 1819 (KATO: 17858).

248. Crataegus microphylla K.Koch

Köprüyanı district, rocky place, $860 \mathrm{~m}$, 08.07.2008, Uzun 1290 (KATO: 17859); Köprüyanı district, open forest, $719 \mathrm{~m}$, 11.07.2008, Uzun 1414 (KATO: 17860); Çeşmeler district, degraded spruce forest, $541 \mathrm{~m}, 06.08 .2008$, Uzun 1820 (KATO: 17861). Hyr.-Eux. el.

\section{Sorbus L. - Üvez}

249. Sorbus aucuparia L. subsp. aucuparia Ormanüstü village, in forest, $1700 \mathrm{~m}$, 05.08.2005, Uzun 777 (KATO: 17862). Euro-Sib. el. 
250. Sorbus torminalis (L.) Crantz var. torminalis

Sukenar1 district, in forest, $663 \mathrm{~m}$, 07.07.2008, Uzun 1151 (KATO: 17863); Köprüyanı district, rocky place, $1017 \mathrm{~m}$, 08.07.2008, Uzun 1291 (KATO: 17864); Ormanüstü village, coniferous-broadleaf mixed forests, $1172 \mathrm{~m}, 10.07 .2008$, Uzun 1375 (KATO: 17865). Euro-Sib. el.

\section{Malus Mill. - Elma}

251. Malus sylvestris (L.) Mill. subsp. orientalis (Uglitzk.) Browicz var. orientalis Çeşmeler district, shrubby place, $437 \mathrm{~m}$, 07.07.2008, Uzun 1152 (KATO: 17866); Köprüyanı district, rocky place, $860 \mathrm{~m}$, 08.07.2008, Uzun 1292 (KATO: 17867).

\section{Pyrus L. - Armut}

252. Pyrus communis L. subsp. communis Sukenar1 district, side of forest, $650 \mathrm{~m}$, 11.07.2007, Uzun 981 (KATO: 17868). Cultivated

\section{F40 [12]. Rhamnaceae Juss.}

\section{Paliurus P.Mill.}

253. Paliurus spina-christi Mill. - Kara çalı Çeşmeler district, stony hills, $435 \mathrm{~m}$, 07.07.2008, Uzun 1180 (KATO: 18002); ibid., 490 m, 07.07.2008, Uzun 1181 (KATO: 18003).

\section{Frangula Mill.}

254. Frangula alnus Mill. subsp. alnus Barut ağac1

Ormanüstü plateau, open forest edge, 1780 m, 05.06.2006, Uzun 913 (KATO: 18004). Euro-Sib. el.

\section{F41 [13]. Ulmaceae Mirb.}

136. Ulmus L. - Karaağaç

255. Ulmus glabra Huds. - Dağ karaağac1

Ormanüstü village, forest edge, $950 \mathrm{~m}$, 08.07.2005, Uzun 622 (KATO: 17567); Köprüyanı district, in forest, $1050 \mathrm{~m}$, 23.05.2006, Uzun 863 (KATO: 17568); Çatak, in forest, $483 \mathrm{~m}, 14.07 .2008$, Uzun 1456 (KATO: 17569). Euro-Sib. el.

256. Ulmus minor Mill. subsp. minor - Ova karaağacı
Çeşmeler district, in forest, $458 \mathrm{~m}$, 05.08.2005, Uzun 762 (KATO: 17570).

\section{F42 [14]. Cannabaceae Martinov}

\section{Celtis L. - Çitlenbik}

257. Celtis australis L. subsp. australis Çitlenbik

Köprüyanı district, forest edge, $580 \mathrm{~m}$, 10.05.2005, Uzun 564 (KATO: 17571). Medit. el.

\section{F43 [15]. Moraceae Gaudich.}

138. Ficus L. - İncir

258. Ficus carica L. subsp. carica

Ormanüstü village, roadside, $929 \mathrm{~m}$, 08.07.2005, Uzun 623 (KATO: 17572).

\section{F44 [16]. Urticaceae Juss.}

\section{Parietaria L.}

259. Parietaria judaica L. - Yapışkan otu, Duvar fesleğeni

Çatak, stony place, $490 \mathrm{~m}, 08.07 .2005$, Uzun 624 (KATO: 17573).

140. Urtica L.

260. Urtica dioica L. subsp. dioica - Isırgan otu

Çeşmeler district, roadside, $600 \mathrm{~m}$, 15.06.2005, Uzun 620 (KATO: 17574). Euro-Sib. el.

Fagales Engl.

F45 [17]. Fagaceae Dumort.

141. Fagus L. - Kayın

261. Fagus orientalis Lipsky - Doğu kayını Ormanüstü village, in forest, $1070 \mathrm{~m}$, 05.08.2005, Uzun 763 (KATO: 17576); ibid., $1400 \mathrm{~m}, 05.08 .2005$, Uzun 764 (KATO: 17577); Köprüyanı district, in forest, $970 \mathrm{~m}$, 11.07.2008, Uzun 1407 (KATO: 17578). Euro-Sib. el.

\section{Castanea Mill. - Kestane}

262. Castanea sativa Mill. - Anadolu kestanesi

Ormanüstü village, in forest, $1300 \mathrm{~m}$, 10.05.2005, Uzun 565 (KATO: 17579). Euro-Sib. el. 
143. Quercus Mill. - Meșe

263. Quercus hartwissiana Steven - Istranca meşesi

Sukenar1 district, in forest, $650 \mathrm{~m}$, 11.07.2007, Uzun 970 (KATO: 17580). Eux. el.

264. Quercus petraea (Matt.) Liebl. subsp. iberica (Steven ex M.Bieb.) Krassiln. Sapsız meşe

Ormanüstü village, in forest, $929 \mathrm{~m}$, 08.07.2005, Uzun 625 (KATO: 17581); Köprüyanı district, in forest, $600 \mathrm{~m}$, 23.05.2006, Uzun 864 (KATO: 17582); Sukenar1 district, in forest, $650 \mathrm{~m}$, 11.07.2007, Uzun 971 (KATO: 17583).

F46 [18]. Juglandaceae DC. ex Perleb

144. Juglans L. - Ceviz

265. Juglans regia L. - Adi ceviz

Sukenar1 village, roadside, $680 \mathrm{~m}$, 08.07.2008, Uzun 1275 (KATO: 17575).

\section{F47 [19]. Betulaceae Gray}

145. Carpinus L. - Gürgen

266. Carpinus betulus L. - Adi gürgen

Ormanüstü village, in forest, $950 \mathrm{~m}$, 08.07.2005, Uzun 626 (KATO: 17584); Ormanüstü village, in forest, $1577 \mathrm{~m}$, 08.07.2005, Uzun 627 (KATO: 17585). Euro-Sib. el.

267. Carpinus orientalis Mill. subsp. orientalis - Doğu gürgeni

Çeşmeler district, in forest, $490 \mathrm{~m}$, 07.07.2008, Uzun 1121 (KATO: 17586); Köprüyanı district, in forest, $860 \mathrm{~m}$, 08.07.2008, Uzun 1276 (KATO: 17587).

\section{Alnus Mill. - Kızılağaç}

268. Alnus glutinosa (L.) Gaertn. subsp. barbata (C.A. Mey.) Yalt. - Sakallı kızılağaç Ormanüstü village, in forest, $1577 \mathrm{~m}$, 08.07.2005, Uzun 628 (KATO: 17588). Eux. el.

\section{Corylus L. - Findık}

269. Corylus avellana L. var. avellana - Adi findik

Ormanüstü village, in forest, $1577 \mathrm{~m}$, 08.07.2005, Uzun 629 (KATO: 17589); ibid., 1067 m, 24.07.2008, Uzun 1734 (KATO: 17590). Euro-Sib. el.
270. Corylus avellana L. var. pontica (K.Koch) H.J.P.Winkl. - Adi findık Köprüyanı district, in forest, $1100 \mathrm{~m}$, 23.05.2006, Uzun 865 (KATO: 17591). Eux. el.

Cucurbitales Juss. ex Bercht. \& J.Presl

F48 [20]. Datiscaceae Dumort.

\section{Datisca L.}

271. Datisca cannabina L. - Renk otu Yukarköy village, shrubby place, $1050 \mathrm{~m}$, 05.08.2005, Uzun 772 (KATO: 17686).

Celastrales Link

F49 [21]. Celastraceae R.Br., nom. cons.

149. Euonymus L. - Papaz Külahı

272. Euonymus latifolius (L.) Mill. subsp. latifolius

Ormanüstü village, in forest, $1133 \mathrm{~m}$, 15.07.2008, Uzun 1552 (KATO: 17981); ibid., $1622 \mathrm{~m}, 15.07 .2008$, Uzun 1553 (KATO: 17982). Euro-Sib. el.

273. Euonymus europaeus L. - İğ ağac1

Ormanüstü village, in forest, $1642 \mathrm{~m}$, 15.07.2008, Uzun 1554 (KATO: 17983).

Oxalidales Bercht. \& J.Presl

F50 [22]. Oxalidaceae R.Br., nom. cons.

150. Oxalis L.

274. Oxalis acetosella L. - Ekşi yonca Ormanüstü village, in forest, $1280 \mathrm{~m}$, 09.07.2008, Uzun 1345 (KATO: 18045).

Malpighiales Juss. ex Bercht. \& J.Presl

F51 [23]. Hypericaceae Juss.

\section{Hypericum L. - Sarı kantaron, Koyun kıran, Binbirdelik otu}

275. Hypericum androsaemum $\mathrm{L}$.

Ormanüstü village, shrubby place, $1050 \mathrm{~m}$, 05.08.2005, Uzun 770 (KATO: 17652; Ormanüstü village, coniferous-broadleaf mixed forests, $1172 \mathrm{~m}, 10.07 .2008$, Uzun 1367 (KATO: 17653.

276. Hypericum bithynicum Boiss.

Çeşmeler district, shrubby place, 08.07.2005, $440 \mathrm{~m}$, Uzun 637 (KATO: 17654); Ormanüstü village, grassy place, $1162 \mathrm{~m}$, 09.07.2008, Uzun 1340 (KATO: 17655); 
ibid, afforestation site, $1862 \mathrm{~m}, 02.08 .2008$, Uzun 1763 (KATO: 17656). Eux. el.

277. Hypericum orientale $\mathrm{L}$.

Ormanüstü village, forest edge, $1750 \mathrm{~m}$, 08.07.2005, Uzun 638 (KATO: 17657); Çeşmeler district, stony hills, $634 \mathrm{~m}$, 07.07.2008, Uzun 1130 (KATO: 17658); Köprüyanı district, open forest, $701 \mathrm{~m}$, 08.07.2008, Uzun 1280 (KATO: 17659); Ormanüstü village, afforestation site, 1683 m, 10.07.2008, Uzun 1368 (KATO: 17660).

278. Hypericum perforatum L. subsp. perforatum

Ormanüstü village, forest-pathside, $929 \mathrm{~m}$, 08.07.2005, Uzun 639 (KATO: 17661); ibid., shrubby place, $1200 \mathrm{~m}, 08.07 .2005$, Uzun 640 (KATO: 17662); Çatak, afforestation site, $873 \mathrm{~m}, 06.08 .2008$, Uzun 1816 (KATO: 17663).

\section{F52 [24]. Violaceae Batsch}

152. Viola L. - Menekşe

279. Viola odorata L. - Kokulu menekşe Ormanüstü village, open forest, $1195 \mathrm{~m}$, 06.08.2008, Uzun 1817 (KATO: 17681).

280. Viola sieheana $\mathrm{W}$.Becker Çatak, in forest, $486 \mathrm{~m}, 14.07 .2008$, Uzun 1457 (KATO: 17682); Ormanüstü village, open forest, $1627 \mathrm{~m}, 15.07 .2008$, Uzun 1544 (KATO: 17683); Çamlıdüz plateau, in forest, 1589 m, 17.07.2008, Uzun 1592 (KATO: 17684).

281. Viola altaica Ker Gawl. subsp. oreades (M.Bieb.) W.Becker

Ormanüstü plateau, grassy place, $1960 \mathrm{~m}$, 10.05.2005, Uzun 568 (KATO: 17685).

\section{F53 [25]. Salicaceae Mirb.}

153. Salix L. - Söğüt

282. Salix alba L. - Ak söğüt

Çeşmeler district, streamside, $490 \mathrm{~m}$, 26.07.2006, Uzun 935 (KATO: 17687). Euro-Sib. el.

283. Salix $\times$ fragilis L. - Gevrek söğüt

Çeşmeler district, streamside, $490 \mathrm{~m}$, 26.07.2006, Uzun 936 (KATO: 17688). Euro-Sib. el.

284. Salix caprea L. - Keçi söğüdü

Yenimahalle, forest edge, $760 \mathrm{~m}, 26.07 .2006$, Uzun 937 (KATO: 17689); Sukenarı district, in forest, $650 \mathrm{~m}$, 11.07.2007, Uzun 974 (KATO: 17690). Euro-Sib. el.

\section{Populus L. - Kavak}

285. Populus tremula L. - Titrek kavak

Ormanüstü village, in forest, $1500 \mathrm{~m}$, 26.07.2006, Uzun 938 (KATO: 17691); Yenimahalle, forest edge, $700 \mathrm{~m}, 26.07 .2006$, Uzun 939 (KATO: 17692). Euro-Sib. el.

286. Populus nigra L. subsp. nigra - Kara kavak

Çeşmeler district, field-side, $490 \mathrm{~m}$, 26.07.2006, Uzun 940 (KATO: 17693).

\section{F54 [26]. Euphorbiaceae Juss.}

\section{Mercurialis L.}

287. Mercurialis annua L. - Yer fesleğeni

Ormanüstü village, open place, $990 \mathrm{~m}$, 05.08.2005, Uzun 783 (KATO: 17986); Çeşmeler district, stony hill, $437 \mathrm{~m}$, 07.07.2008, Uzun 1175 (KATO: 17987).

\section{Euphorbia L. - Sütleğen}

*288. Euphorbia chamaesyce L.

Çeşmeler district, stony hill, $490 \mathrm{~m}$, 07.07.2008, Uzun 1176 (KATO: 17988).

289. Euphorbia stricta L.

Ormanüstü plateau, roadside, $1800 \mathrm{~m}$, 10.05.2005, Uzun 579 (KATO: 17989); Köprüyan1 district, roadside, $890 \mathrm{~m}$, 11.07.2007, Uzun 988 (KATO: 17990). Euro-Sib. el.

290. Euphorbia peplus L.

Çeşmeler district, stony hill, $583 \mathrm{~m}$, 07.07.2008, Uzun 1177 (KATO: 17991).

291. Euphorbia falcata L. subsp. falcata var. falcata

Çeşmeler district, stony hill, $437 \mathrm{~m}$, 07.07.2008, Uzun 1178 (KATO: 17992); Köprüyan1 district, roadside, $890 \mathrm{~m}$, 11.07.2007, Uzun 989 (KATO: 17993).

292. Euphorbia herniariifolia Willd. var. glaberrima Halácsy

Kiremitli, roadside stony place, $717 \mathrm{~m}$, 13.07.2007, Uzun 1035 (KATO: 17994); Çeşmeler, roadside stony place, $437 \mathrm{~m}$, 07.07.2008, Uzun 1179 (KATO: 17995).

293. Euphorbia oblongifolia (K.Koch) K.Koch

Ormanüstü village, in forest, $1850 \mathrm{~m}$, 08.07.2005, Uzun 678 (KATO: 17996); Ormanüstü village, open forest, $1350 \mathrm{~m}$, 
08.07.2005, Uzun 679 (KATO: 17997); Çamlıdüz plateau, in forest, $1720 \mathrm{~m}$, 17.07.2008, Uzun 1602 (KATO: 17998); Çamlıdüz plateau, afforestation site, $1728 \mathrm{~m}$, 20.07.2008, Uzun 1653 (KATO: 17999); Çeşmeler district, degraded spruce forest, $541 \mathrm{~m}, 06.08 .2008$, Uzun 1825 (KATO: 18000). Eux. el.

294. Euphorbia amygdaloides L. subsp. amygdaloides

Çatak, afforestation site, $477 \mathrm{~m}, 14.07 .2008$, Uzun 1477 (KATO: 18001). Euro-Sib. el.

\section{F55 [27]. Linaceae DC. ex Perleb}

\section{Linum L. - Keten otu}

295. Linum corymbulosum Rchb.

Çeşmeler district, stony-grassy place, $490 \mathrm{~m}$, 07.07.2008, Uzun 1182 (KATO: 18006); ibid., 583 m, 07.07.2008, Uzun 1183 (KATO: 18007); Çeşmeler district, stony hill, $632 \mathrm{~m}$, 11.07.2008, Uzun 1423 (KATO: 18008); Çatak, afforestation site, $477 \mathrm{~m}, 14.07 .2008$, Uzun 1478 (KATO: 18009). Medit. el.

296. Linum aroanium Boiss. \& Orph.

Çeşmeler district, stony hill, $880 \mathrm{~m}$, 18.06.2008, Uzun 1100 (KATO: 18010).

297. Linum tenuifolium $\mathrm{L}$.

Çeşmeler district, stony hill, $750 \mathrm{~m}$, 01.06.2008, Uzun 1065 (KATO: 18011); ibid., 490 m, 07.07.2008, Uzun 1184 (KATO: 18012); Çatak, afforestation site, $873 \mathrm{~m}$, 06.08.2008, Uzun 1826 (KATO: 18013).

\section{F56 [28]. Phyllanthaceae Martinov}

\section{Andrachne L.}

298. Andrachne telephioides $\mathrm{L}$.

Çeşmeler district, stony hill, $437 \mathrm{~m}$, 07.07.2008, Uzun 1174 (KATO: 17985).

Geraniales Juss. ex Bercht. \& J.Presl

F57 [29]. Geraniaceae Juss.

\section{Geranium L. - Turna gagası}

299. Geranium robertianum L.

Köprüyanı district, stony place, $1130 \mathrm{~m}$, 23.05.2006, Uzun 879 (KATO: 18046).

300. Geranium columbinum L.

Çeşmeler district, stony hill, $632 \mathrm{~m}$, 11.07.2008, Uzun 1424 (KATO: 18047).

301. Geranium psilostemon Ledeb.
Ormanüstü village, forest edge, $1280 \mathrm{~m}$, 09.07.2008, Uzun 1346 (KATO: 18048).

Eux. (mt.) el.

302. Geranium sylvaticum L.

Ormanüstü village, forest edge, $1770 \mathrm{~m}$, 08.07.2005, Uzun 683 (KATO: 18049); Çamlıdüz plateau, in forest, $1589 \mathrm{~m}$, 17.07.2008, Uzun 1603 (KATO: 18050); Çamlıdüz plateau, afforestation site, $1728 \mathrm{~m}$, 20.07.2008, Uzun 1654 (KATO: 18051). Euro-Sib. el.

303. Geranium sintenisii Freyn (syn.: Geranium asphodeloides Burm. fil. subsp. sintenisii (Freyn) P.H.Davis)

Çamlıdüz plateau, afforestation site, $1728 \mathrm{~m}$, 20.07.2008, Uzun 1655 (KATO: 18052). Eux. el., Endemic, IUCN: LC

304. Geranium sanguineum $\mathrm{L}$.

Sukenar1 district, in forest, $663 \mathrm{~m}$, 07.07.2008, Uzun 1188 (KATO: 18053); Çeşmeler district, stony hill, $942 \mathrm{~m}$, 08.07.2008, Uzun 1302 (KATO: 18054). Euro-Sib. el.

305. Geranium pyrenaicum Burm.f. subsp. pyrenaicum

Ormanüstü village, roadside, $1350 \mathrm{~m}$, 10.05.2005, Uzun 581 (KATO: 18055).

306. Geranium gracile Ledeb. ex Nordm. Ormanüstü village, forest edge, $950 \mathrm{~m}$, 08.07.2005, Uzun 684 (KATO: 18056); Ormanüstü village, coniferous-broadleaf mixed forests, $1172 \mathrm{~m}, 10.07 .2008$, Uzun 1379 (KATO: 18057); Ormanüstü village, open forest, $1627 \mathrm{~m}, 15.07 .2008$, Uzun 1556 (KATO: 18058); Ormanüstü plateau, afforestation site, $1664 \mathrm{~m}, 21.07 .2008$, Uzun 1690 (KATO: 18059). Hyr.-Eux. el.

307. Geranium ibericum Cav. subsp. jubatum (Hand.-Mazz.) P.H.Davis

Ormanüstü plateau, shrubby place, $1850 \mathrm{~m}$, 10.05.2005, Uzun 582 (KATO: 18060). Eux. (mt.) el., Endemic, IUCN: LC

\section{Erodium L'Hér. - İğnelik otu}

308. Erodium cicutarium (L.) L Hér. subsp. cicutarium

Çeşmeler district, roadside, $700 \mathrm{~m}$, 01.06.2008, Uzun 1068 (KATO: 18061); Çeşmeler district, roadside, $900 \mathrm{~m}$, 18.06.2008, Uzun 1103 (KATO: 18062). 
Myrtales Juss. ex Bercht. \& J.Presl

F58 [30]. Lythraceae J.St.-Hil.

161. Lythrum L.

309. Lythrum salicaria L. - Aklar ot Ormanüstü village, damp creekside, $1200 \mathrm{~m}$, 05.06.2006, Uzun 911 (KATO: 17965). Euro-Sib. el.

\section{Punica L.}

310. Punica granatum L. - Nar

Köprüyan1, Carpinus orientalis thicket, 650 m, 11.07.2008, Uzun 1421 (KATO: 17968). Medit. el.

\section{F59 [31]. Onagraceae Juss.}

\section{Circaea L.}

311. Circaea lutetiana $\mathrm{L}$.

Ormanüstü village, damp places in forest, $1050 \mathrm{~m}, 05.08 .2005$, Uzun 781 (KATO: 17969).

312. Circaea alpina $\mathrm{L}$.

Ormanüstü village, forest edge, $1245 \mathrm{~m}$, 13.08.2005, Uzun 856 (KATO: 17970).

\section{Chamaenerion Adans.}

313. Chamaenerion angustifolium (L.) Scop. (syn.: Epilobium angustifolium L.) - Yak1 otu

Ormanüstü village, damp hill, $1300 \mathrm{~m}$, 08.07.2005, Uzun 676 (KATO: 17971).

\section{Epilobium L.}

314. Epilobium hirsutum L.

Ormanüstü village, damp creekside, $980 \mathrm{~m}$, 05.06.2006, Uzun 912 (KATO: 17972).

315. Epilobium parviflorum Schreb.

Sukenar1 district, roadside, $640 \mathrm{~m}$, 07.07.2008, Uzun 1170 (KATO: 17973).

316. Epilobium montanum L.

Çamlıdüz plateau, afforestation site, $1728 \mathrm{~m}$, 20.07.2008, Uzun 1650 (KATO: 17974); ibid., in forest, $1786 \mathrm{~m}, 20.07 .2008$, Uzun 1651 (KATO: 17975). Euro-Sib. el.

317 Epilobium lanceolatum Sebast. \& Mauri Ormanüstü village, roadside, $1750 \mathrm{~m}$, 05.08.2005, Uzun 782 (KATO: 17976).
Crossosomatales Takht. ex Reveal

F60 [32]. Staphyleaceae Martinov

166. Staphylea L.

318. Staphylea pinnata L. - Patlak

Köprüyan1 district, forest edge, $1200 \mathrm{~m}$, 11.07.2007, Uzun 990 (KATO: 18022); Çatak, in forest, $486 \mathrm{~m}, 14.07 .2008$, Uzun 1581 (KATO: 18023); Çeşmeler district, in forest, $529 \mathrm{~m}$, 14.07.2008, Uzun 1775 (KATO: 18024).

Sapindales Juss. ex Bercht. \& J.Presl

F61 [33]. Anacardiaceae R.Br.

167. Rhus L. - Sumak

319. Rhus coriaria L. - Derici sumağ1

Çeşmeler district, shrubby place, $600 \mathrm{~m}$, 01.06.2008, Uzun 1066 (KATO: 18037).

\section{Pistacia L.}

320. Pistacia palaestina Boiss. (syn.: Pistacia terebinthus L. subsp. palaestina (Boiss.) Engler) - Menengiç

Çeşmeler district, shrubby place, $415 \mathrm{~m}$, 05.08.2005, Uzun 785 (KATO: 18039); Karşıyaka village, roadside, $810 \mathrm{~m}$, 13.07.2007, Uzun 1036 (KATO: 18038); Çeşmeler district, shrubby place, $583 \mathrm{~m}$, 07.07.2008, Uzun 1186 (KATO: 18040). Medit. el.

F62 [34]. Sapindaceae Juss., (including Xanthocerataceae Buerki et al., as 'Xanthoceraceae')

169. Acer Desf. - Akçaağaç

321. Acer heldreichii Orph. subsp. trautvetteri (Medw.) A.E.Murray (syn.: Acer trautvetteri Medw.) - Kayin gövdeli akçaağaç

Ormanüstü village, in forest, $1680 \mathrm{~m}$, 08.07.2005, Uzun 681 (KATO: 18025); Köprüyan1 district, in forest, $1000 \mathrm{~m}$, 23.05.2006, Uzun 877 (KATO: 18026). Eux. el.

322. Acer cappadocicum Gled. subsp. cappadocicum - Doğu Karadeniz akçaağacı Ormanüstü village, leafy forest interior, 1250 m, 10.05.2005, Uzun 580 (KATO: 18027); Köprüyan1, associated with Quercus petraea, 910 m, 23.05.2006, Uzun 878 (KATO: 
18028); Sukenar1 district, forest edge, $650 \mathrm{~m}$, 11.07.2007, Uzun 991 (KATO: 18029); Ormanüstü village, in forest, $1133 \mathrm{~m}$, 15.07.2008, Uzun 1555 (KATO: 18030). Hyr.-Eux. el.

323. Acer platanoides L. - Çınar yapraklı akçaağaç

Ormanüstü village, in forest, $1204 \mathrm{~m}$, 24.07.2008, Uzun 1739 (KATO: 18031).

Euro-Sib. el.

324. Acer campestre L. subsp. campestreOva akçaağacı

Ormanüstü village, in forest, $929 \mathrm{~m}$, 08.07.2005, Uzun 682 (KATO: 18032); Köprüyan1 district, forest edge, $1200 \mathrm{~m}$, 11.07.2007, Uzun 992 (KATO: 18033); Kiremitli, in forest, 702 m, 23.07.2008, Uzun 1726 (KATO: 18034).

325. Acer campestre L. subsp. leiocarpum (Opiz) Schwer. - Ova akçaağacı

Sukenar1 district, shrubby place, $663 \mathrm{~m}$, 07.07.2008, Uzun 1185 (KATO: 18035); Çeşmeler district, stony hill, $942 \mathrm{~m}$, 08.07.2008, Uzun 1301 (KATO: 18036). Euro-Sib. el.

\section{F63 [35]. Rutaceae Juss.}

170. Dictamnus L.

326. Dictamnus albus L. - Gazel otu, Geyik otu

Çeşmeler district, with Carpinus orientalis, $750 \mathrm{~m}$, 01.06.2008, Uzun 1067 (KATO: 18042); ibid., 890 m, 18.06.2008, Uzun 1102 (KATO: 18043); Sukenar1 district, with Carpinus orientalis, $663 \mathrm{~m}$, 07.07.2008, Uzun 1187 (KATO: 18044).

\section{F64 [36]. Simaroubaceae DC.}

171. Ailanthus Desf.

327. Ailanthus altissima (Mill.) Swingle Cennet ağac1, Kokar ağaç

Karşıyaka village, roadside, $810 \mathrm{~m}$, 13.07.2007, Uzun 1037 (KATO: 18041). Exotic

Malvales Juss. ex Bercht. \& J.Presl

F65 [37]. Malvaceae Juss.

172. Tilia L. - Ihlamur

328. Tilia platyphyllos Scop. subsp. platyphyllos - Büyük yapraklı 1hlamur
Ormanüstü village, in forest, $1085 \mathrm{~m}$, 10.07.2008, Uzun 1369 (KATO: 17664). Euro-Sib. el.

173. Malva L. - Ebegümeci

329. Malva sylvestris L. - Büyük ebegümeci Çeşmeler district, open place, $440 \mathrm{~m}$, 08.07.2005, Uzun 641 (KATO: 17665); Ormanüstü village, open place, $993 \mathrm{~m}$, 05.08.2005, Uzun 771 (KATO: 17666).

330. Malva neglecta Wallr.

Köprüyan1, open place, 1200 m, 11.07.2007, Uzun 973 (KATO: 17667).

\section{Alcea L.}

331. Alcea hohenackeri (Boiss. \& A. Huet) Boiss.

Sindıran, stony landslides, hills, $800 \mathrm{~m}$, 26.07.2008, Uzun 1749 (KATO: 17668).

332. Alcea biennis Winterl subsp. biennis (syn.: Alcea pallida (Willd.) \& Kit.)

Çatak, roadside, $550 \mathrm{~m}, 26.07 .2008$, Uzun 1750 (KATO: 17669).

\section{F66 [38]. Thymelaeaceae Juss.}

175. Daphne L. - Dafne

333. Daphne glomerata Lam.

Ormanüstü plateau, degraded spruce forest, 1991 m, 02.08.2008, Uzun 1774 (KATO: 17966). Eux. el.

334. Daphne pontica L. subsp. pontica Sırımağu

Çeşmeler district, in forest, $1020 \mathrm{~m}$, 08.07.2008, Uzun 1300 (KATO: 17967). Eux. el.

\section{F67 [39]. Cistaceae Juss.}

176. Cistus L. - Laden

335. Cistus creticus L. subsp. creticus Pembe çiçekli laden

Köprüyanı district, roadside slope, $600 \mathrm{~m}$, 23.05.2006, Uzun 866 (KATO: 17670); Köprüyanı district, rocky place, $860 \mathrm{~m}$, 08.07.2008, Uzun 1281 (KATO: 17671). Medit. el.

\section{Helianthemum Adans.}

336. Helianthemum nummularium (L.) Mill. subsp. nummularium

Ormanüstü plateau, open place, $2000 \mathrm{~m}$, 05.06.2006, Uzun 897 (KATO: 17672); 
Çeşmeler district, stony hill, $583 \mathrm{~m}$, 07.07.2008, Uzun 1131 (KATO: 17673); Çeşmeler district, stony hills, $634 \mathrm{~m}$, 07.07.2008, Uzun 1132 (KATO: 17674); Ormanüstü plateau, afforestation site, 1862 m, 02.08.2008, Uzun 1764 (KATO: 17675).

337. Helianthemum tomentosum (Scop.) Gray subsp. tomentosum (syn.: Helianthemum nummularium (L.) Mill. subsp. tomentosum (Scop.) Schinz \& Thell.) Ormanüstü village, forest edge, $929 \mathrm{~m}$, 08.07.2005, Uzun 643 (KATO: 17676); Çeşmeler district, stony hills, $750 \mathrm{~m}$, 01.06.2008, Uzun 1054 (KATO: 17677).

\section{Fumana Spach}

338. Fumana procumbens (Dunal) Gren. \& Godr.

Çeşmeler district, shrubby place, $750 \mathrm{~m}$, 01.06.2008, Uzun 1055 (KATO: 17678); Çeşmeler district, stony place, $880 \mathrm{~m}$, 18.06.2008, Uzun 1094 (KATO: 17679); Çeşmeler district, stony hill, $437 \mathrm{~m}$, 07.07.2008, Uzun 1133 (KATO: 17680).

Brassicales Bromhead

\section{F68 [40]. Resedaceae Martinov}

179. Reseda L. - Sarı muhabbet çiçeği 339. Reseda lutea L. var. lutea - Gerdanlık Çeşmeler district, stony place, $700 \mathrm{~m}$, 01.06.2008, Uzun 1059 (KATO: 17720); ibid., 490 m, 07.07.2008, Uzun 1138 (KATO: 17721).

F69 [41]. Brassicaceae Burnett (= Cruciferae Juss.)

180. Sinapis L.

340. Sinapis alba L. subsp. alba

Çeşmeler village, damp road-side, $490 \mathrm{~m}$, 05.06.2006, Uzun 898 (KATO: 17694).

\section{Raphanus L.}

341. Raphanus raphanistrum L. subsp. raphanistrum

Çeşmeler district, stony hill, $750 \mathrm{~m}$, 12.07.2007, Uzun 1024 (KATO: 17695).

\section{Lepidium L.}

342. Lepidium campestre (L.) W.T.Aiton Çeşmeler district, stony hill, $437 \mathrm{~m}$, 07.07.2008, Uzun 1134 (KATO: 17696).
183. Aethionema W.T.Aiton

*343. Aethionema diastrophis Bunge

Çeşmeler district, stony hills, $437 \mathrm{~m}$, 07.07.2008, Uzun 1135 (KATO: 17697).

184. Microthlaspi F.K.Mey.

344. Microthlaspi perfoliatum (L.) F.K.Mey. (syn.: Thlaspi perfoliatum L.)

Çeşmeler district, stony roadside, $750 \mathrm{~m}$, 01.06.2008, Uzun 1056 (KATO: 17698).

185. Pachyphragma Rchb.

345. Pachyphragma macrophyllum (Hoffm.) N.Busch

Ormanüstü village, roadside, $1330 \mathrm{~m}$, 10.05.2005, Uzun 569 (KATO: 17699). Eux. el.

186. Capsella Medik. - Çoban çantası

346. Capsella bursa-pastoris (L.) Medik. subsp. bursa-pastoris - Kuşkuş otu

Ormanüstü village, damp creekside, $1200 \mathrm{~m}$, 05.06.2006, Uzun 899 (KATO: 17700).

Cosmopolitan

\section{Alyssoides Adans}

347. Alyssoides utriculata (L.) Medik. subsp. utriculata

Kiremitli, rock cavities, 760-900 m, 13.07.2007, Uzun 1032 (KATO: 17701).

188. Fibigia Medik.

348. Fibigia clypeata (L.) Medik. subsp. clypeata var. clypeata

Çeşmeler district, rocky roadside slope, 650 m, 11.07.2008, Uzun 1409 (KATO: 17702).

189. Berteroa DC.

349. Berteroa mutabilis (Vent.) DC.

Çatak, stony hill, $400 \mathrm{~m}, 11.07 .2007$, Uzun 975 (KATO: 17703); Kiremitli, stony hill, 760-900 m, 13.07.2007, Uzun 1033 (KATO: 17704).

190. Alyssum L.

350. Alyssum alyssoides (L.) L.

Çeşmeler district, roadside, $700 \mathrm{~m}$, 01.06.2008, Uzun 1057 (KATO: 17705).

351. Alyssum simplex Rudolph (syn.: Alyssum minus (L.) Rothm. var. minus)

Çeşmeler district, roadside, $700 \mathrm{~m}$, 01.06.2008, Uzun 1058 (KATO: 17706); Çeşmeler district, stony hill, $634 \mathrm{~m}$, 
07.07.2008, Uzun 1136 (KATO: 17707); Çeşmeler district, stony hill, $632 \mathrm{~m}$, 11.07.2008, Uzun 1410 (KATO: 17708).

\section{Draba L.}

352. Draba hispida Willd.

Çeşmeler district, stony hill, $632 \mathrm{~m}$, 11.07.2008, Uzun 1411 (KATO: 17709). Eux. (mt.) el.

\section{Arabis L.}

*353. Arabis sagittata (Bertol.) DC.

Çatak, afforestation site, $477 \mathrm{~m}, 14.07 .2008$, Uzun 1458 (KATO: 17710).

193. Turritis L.

354. Turritis glabra L. (syn.: Arabis glabra L.)

Ormanüstü village, roadside, $490 \mathrm{~m}$, 07.07.2008, Uzun 1137 (KATO: 17711).

\section{Nasturtium W.T.Aiton}

355. Nasturtium officinale W.T.Aiton - Su teresi

Sukenarı district, damp creekside, $632 \mathrm{~m}$, 11.07.2008, Uzun 1412 (KATO: 17712).

\section{Cardamine $\mathbf{L}$.}

356. Cardamine bulbifera (L.) Crantz

Ormanüstü village, in forest, $1647 \mathrm{~m}$, 15.07.2008, Uzun 1545 (KATO: 17713). Euro-Sib. el.

357. Cardamine quinquefolia (M.Bieb.) Benth. \& Hook.f. ex Schmalh.

Ormanüstü village, in forest, $1642 \mathrm{~m}$, 15.07.2008, Uzun 1546 (KATO: 17714). Euro-Sib. el.

358. Cardamine acris Griseb. (syn.: Cardamine raphanifolia Pourr. subsp. acris (Griseb.) O.E.Schulz)

Ormanüstü village, damp hills, $1100 \mathrm{~m}$, 10.05.2005, Uzun 570 (KATO: 17715). Euro-Sib. el.

359. Cardamine impatiens $\mathrm{L}$.

Ormanüstü village, damp hills, $1100 \mathrm{~m}$, 10.05.2005, Uzun 571 (KATO: 17716). Euro-Sib. el.

360. Cardamine pectinata DC. (syn.: Cardamine impatiens L. subsp. pectinata (DC.) Stoj. \& Stef.)

Ormanüstü village, forest edge, $1370 \mathrm{~m}$, 10.05.2005, Uzun 572 (KATO: 17717). Euro-Sib. el.
196. Alliaria Scop.

361. Alliaria petiolata (M.Bieb.) Cavara \& Grande - Sarımsak otu

Ormanüstü village, open forest edge, $1370 \mathrm{~m}$, 10.05.2005, Uzun 573 (KATO: 17718).

197. Sisymbrium L.

362. Sisymbrium officinale (L.) Scop.

Köprüyan1, roadside, 870 m, 23.05.2006, Uzun 867 (KATO: 17719).

\section{SUPERASTERIDS}

Santalales R.Br. ex Bercht. \& J.Presl

F70 [42]. Santalaceae R.Br.

\section{Thesium L.}

*363. Thesium billardieri Boiss.

Çeşmeler district, stony hills, $490 \mathrm{~m}$, 07.07.2008, Uzun 1172 (KATO: 17979); ibid., 583 m, 07.07.2008, Uzun 1173 (KATO: 17980). Ir.-Tur. el.

Caryophyllales Juss. ex Bercht. \& J.Presl F71 [43]. Polygonaceae Juss.

199. Polygonum L. - Çobandeğneği

364. Polygonum aviculare L.

Sukenarı district, damp place, $640 \mathrm{~m}$, 07.07.2008, Uzun 1128 (KATO: 17647). Cosmopolitan

\section{Bistorta L. (Scop.)}

365. Bistorta carneum (K.Koch) Kom. ex Tzvelev (syn.: Polygonum bistorta L. subsp. carneum (K.Koch) Coode \& Cullen)

Ormanüstü plateau, wet meadows, $1815 \mathrm{~m}$, 08.07.2005, Uzun 636 (KATO: 17643); Ormanüstü plateau, grassy place, $2020 \mathrm{~m}$, 05.08.2005, Uzun 769 (KATO: 17644); ibid., $2000 \mathrm{~m}$, 05.06.2006, Uzun 896 (KATO: 17645). Eux (mt.) el.

\section{Persicaria Mill.}

366. Persicaria maculosa Gray (syn.: Polygonum persicaria L.)

Sukenarı district, damp place, $640 \mathrm{~m}$, 07.07.2008, Uzun 1127 (KATO: 17646).

202. Rumex L. - Kuzu kulağı

367. Rumex arifolius All.

Ormanüstü plateau, wet meadows, $1850 \mathrm{~m}$, 10.05.2005, Uzun 567 (KATO: 17648. 
368. Rumex crispus $\mathrm{L}$.

Çeşmeler, damp place, $437 \mathrm{~m}, 07.07 .2008$, Uzun 1129 (KATO: 17649); Ormanüstü village, damp place, $1467 \mathrm{~m}, 12.07 .2008$, Uzun 1444 (KATO: 17650).

369. Rumex pulcher L. subsp. pulcher

Sukenar1 district, damp creekside, $640 \mathrm{~m}$, 07.07.2008, Uzun 1272 (KATO: 17651).

\section{F72 [44]. Caryophyllaceae Juss.}

\section{Arenaria L.}

370. Arenaria kotschyana Frenzl subsp. kotschyana

Ormanüstü plateau, in the cracks of rock clusters, $2000 \mathrm{~m}$, 16.07.2008, Uzun 1575 (KATO: 17593). Endemic, IUCN: LC

371. Arenaria rotundifolia M.Bieb. subsp. rotundifolia

Ormanüstü plateau, rocky place, $2000 \mathrm{~m}$, 05.06.2006, Uzun 894 (KATO: 17594).

372. Arenaria serpyllifolia $\mathrm{L}$.

Çeşmeler district, stony hill, $583 \mathrm{~m}$, 07.07.2008, Uzun 1123 (KATO: 17595).

\section{Cherleria L.}

373. Cherleria circassica (Albov) A.J. Moore \& Dillenb. (syn.: Minuartia circassica (Albow) Woronow)

Ormanüstü plateau, in cracks of rock clusters, 2000 m, 16.07.2008, Uzun 1576 (KATO: 17596).

Eux. (mt.) el.

\section{Moehringia L.}

374. Moehringia trinervia (L.) Clairv. Ormanüstü village, in forest, $1948 \mathrm{~m}$, 22.07.2008, Uzun 1708 (KATO: 17597); Vicinity of Vazelon monastry, in forest, 1069 m, 24.07.2008, Uzun 1735 (KATO: 17598).

\section{Stellaria L. - Kuş otu}

375. Stellaria media L. subsp. media Çamlıdüz plateau, damp places in forest, 1879 m, 20.07.2008, Uzun 1638 (KATO: 17599).

*376. Stellaria nemorum L. subsp. nemorum Çamlıdüz plateau, in forest, $1576 \mathrm{~m}$, 17.07.2008, Uzun 1590 (KATO: 17600); ibid., 1589 m, 17.07.2008, Uzun 1591 (KATO: 17601); ibid., 1786 m, 20.07.2008, Uzun 1639 (KATO: 17602); ibid., 1769 m, 22.07.2008, Uzun 1709 (KATO: 17603).
207. Rabelera M.T.Sharples \& E.A.Tripp 377. Rabelera holostea (L.) M.T.Sharples \& E.A.Tripp (syn.: Stellaria holostea L.)

Vicinity of Vazelon monastry, forest edge, 1200 m, 24.07.2008, Uzun 1736 (KATO: 17604). Euro-Sib. el.

208. Cerastium L.

378. Cerastium dubium (Bastard) Guépin (syn.: Cerastium anomalum Wadst. \& Kit.) Ormanüstü village, open place, $1680 \mathrm{~m}$, 08.07.2005, Uzun 758 (KATO: 17605).

379. Cerastium armeniacum Gren

Ormanüstü plateau, grassy place, $2000 \mathrm{~m}$, 05.06.2006, Uzun 895 (KATO: 17606).

Endemic, IUCN: LC

380. Cerastium fontanum Baumg. subsp. vulgare (Hartm.) Greuter \& Burdet (syn.: Cerastium fontanum Baumg. subsp. triviale (Link) Jalas)

Ormanüstü plateau, grassy place, 1945 m, 08.07.2005, Uzun 630 (KATO: 17607). Cosmopolitan

381. Cerastium purpurascens Adams

Ormanüstü plateau, grassy place, $1945 \mathrm{~m}$, 08.07.2005, Uzun 631 (KATO: 17608); ibid., $1900 \mathrm{~m}, 05.08 .2005$, Uzun 765 (KATO: 17609).

\section{Moenchia Ehrh.}

382. Moenchia mantica (L.) Bartl. subsp. mantica

Ormanüstü plateau, grassy place, $1815 \mathrm{~m}$, 08.07.2005, Uzun 632 (KATO: 17610); Kiremitli, grassy place, 760-900 m, 13.07.2007, Uzun 1030 (KATO: 17611); Ormanüstü village, grassy place, $1162 \mathrm{~m}$, 09.07.2008, Uzun 1338 (KATO: 17613).

\section{Dianthus L. - Karanfil \\ 383. Dianthus sp.}

Ormanüstü plateau, grassy place, $1900 \mathrm{~m}$, 01.06.2008, Uzun 1053 (KATO: 17614).

Note: It was a sample that was highly affected by excessive grazing pressure. Therefore, we could not describe it.

384. Dianthus carmelitarum Reut. ex Boiss. Çeşmeler district, grassy place, $444 \mathrm{~m}$, 08.07.2005, Uzun 633 (KATO: 17615); Kiremitli district, roadside slope, 760-900 m, 13.07.2007, Uzun 1031 (KATO: 17616); Sukenar1 district, stony hill, $663 \mathrm{~m}$, 07.07.2008, Uzun 1124 (KATO: 17619); 
Çeşmeler district, stony hill, $632 \mathrm{~m}$, 11.07.2008, Uzun 1277 (KATO: 17621); Çatak, afforestation site, $873 \mathrm{~m}, 06.08 .2008$, Uzun 1815 (KATO: 17624). Eux. el., Endemic, IUCN: LC

\section{Petrorhagia (Ser.) Link.}

385. Petrorhagia saxifraga (L.) Link

Çeşmeler district, stony hill, $1050 \mathrm{~m}$, 11.07.2007, Uzun 972 (KATO: 17625); Çeşmeler district, stony hill, $437 \mathrm{~m}$, 07.07.2008, Uzun 1125 (KATO: 17626); ibid. 634 m, 07.07.2008, Uzun 1126 (KATO: 17627); ibid., 632 m, 11.07.2008, Uzun 1408 (KATO: 17628). Euro-Sib. el.

\section{Silene L. - Salkım çiçeği}

386. Silene italica (L.) Pers. subsp. italica Çamlıdüz plateau, afforestation site, $1728 \mathrm{~m}$, 20.07.2008, Uzun 1640 (KATO: 17629).

387. Silene saxatilis Sims

Ormanüstü village, rocky place, $1577 \mathrm{~m}$, 08.07.2005, Uzun 634 (KATO: 17630); Ormanüstü plateau, degraded oriental spruce forest, $1991 \mathrm{~m}$, 02.08.2008, Uzun 1762 (KATO: 17631); Ormanüstü village, rocky hill, 1750 m, 05.08.2005, Uzun 766 (KATO: 17632).

388. Silene vulgaris (Moench) Garcke subsp. vulgaris - Gıvışkan otu

Ormanüstü village, roadside slope, open place, 929 m, 08.07.2005, Uzun 635 (KATO: 17633); Ormanüstü village, grassy place, 1162 m, 09.07.2008, Uzun 1339 (KATO: 17634); Ormanüstü village, open place, 1740 m, 16.07.2008, Uzun 1577 (KATO: 17635); Çamlıdüz plateau, afforestation site, $1728 \mathrm{~m}$, 20.07.2008, Uzun 1641 (KATO: 17636).

389. Silene latifolia Poir. (syn.: Silene latifolia Poir. subsp. alba (Mill.) Greuter \& Burdet, Silene alba (Mill.) Krause subsp. divaricata (Reichb.) Walters) - G1c1 gic1 otu Ormanüstü village, shrubby place, $1500 \mathrm{~m}$, 05.08.2005, Uzun 768 (KATO: 17640); Köprüyanı district, open forest, $975 \mathrm{~m}$, 08.07.2008, Uzun 1278 (KATO: 17641).

390. Silene gallica $\mathrm{L}$.

Köprüyanı district, open forest, $975 \mathrm{~m}$, 08.07.2008, Uzun 1279 (KATO: 17642). Cosmopolitan
213. Atocion Adans.

391. Atocion compactum (Fisch. ex Hornem.) Tzvelev (syn.: Silene compacta Fischer ex Hornem.)

Ormanüstü village, forest edge open place, $1200 \mathrm{~m}, 10.05 .2005$, Uzun 566 (KATO: 17637); Ormanüstü village, open place, 1620 m, 05.08.2005, Uzun 767 (KATO: 17639). ibid., 1615 m, 26.07.2006, Uzun 934 (KATO: 17638).

\section{F73 [45]. Cactaceae Juss.}

\section{Opuntia Mill.}

*392. Opuntia ficus-indica (L.) Mill. - Frenk inciri

Çeşmeler district, stony-open place, $490 \mathrm{~m}$, 07.07.2008, Uzun 1122 (KATO: 17592). Exotic

\section{ASTERIDS \\ Cornales Link \\ F74 [46]. Cornaceae Bercht. \& J.Presl}

\section{Cornus L. - Kızılcık}

393. Cornus sanguinea L. subsp. australis (C.A.Mey.) Jáv.

Ormanüstü village, forest edge, $950 \mathrm{~m}$, 08.07.2005, Uzun 677 (KATO: 17977). Euro-Sib. el.

394. Cornus mas L.

Çeşmeler district, shrubby place, $634 \mathrm{~m}$, 07.07.2008, Uzun 1171 (KATO: 17978). Euro-Sib. el.

Ericales Bercht. \& J.Presl

F75 [47]. Balsaminaceae A.Rich.

\section{Impatiens L.}

395. Impatiens noli-tangere L. - Kına çiçeği Ormanüstü village, in forest, $1500 \mathrm{~m}$, 10.05.2005, Uzun 583 (KATO: 18063); ibid., $1091 \mathrm{~m}, 05.08 .2005$, Uzun 786 (KATO: 18064); Ormanüstü village, damp places in forest, $1350 \mathrm{~m}$, 26.07.2006, Uzun 946 (KATO: 18065). Euro-Sib. el.

F76 [48]. Primulaceae Batsch ex Borkh.

217. Primula L. - Çuha çiçeği

396. Primula acaulis (L.) L. subsp. acaulis (syn.: Primula vulgaris Huds. subsp. vulgaris) 
Sukenar1 district, in forest, $663 \mathrm{~m}$, 07.07.2008, Uzun 1139 (KATO: 17740).

Euro-Sib. el.

397. Primula acaulis (L.) L. subsp. rubra (Sm.) Greuter \& Burdet (syn.: Primula vulgaris Huds. subsp. sibthorpii (Hoffmanns.) W.W.Sm. \& Forrest)

Çeşmeler district, open place, $500 \mathrm{~m}$, 07.07.2008, Uzun 1140 (KATO: 17741). Eux. el.

398. Primula veris L. subsp. columnae (Ten.) Maire \& Petitm.

Ormanüstü plateau, grassy hill, $1800 \mathrm{~m}$, 05.06.2006, Uzun 900 (KATO: 17742).

Euro-Sib. el.

399. Primula auriculata Lam.

Ormanüstü Y., damp creekside, $2000 \mathrm{~m}$, 05.06.2006, Uzun 901 (KATO: 17743). Ir.Tur. el.

\section{Cyclamen L. - Domuzağırşağı}

400. Cyclamen coum Mill. subsp. coum

Çeşmeler district, stony place, $490 \mathrm{~m}$, 10.04.2005, Uzun 553 (KATO: 17744).

401. Cyclamen coum Mill. subsp. caucasicum (K. Koch) O. Schwarz

Sukenar1 district, stony place, $670 \mathrm{~m}$, 10.04.2005, Uzun 554 (KATO: 17745).

402. Cyclamen parviflorum Pobed. subsp. parviflorum

Ormanüstü plateau, stony place, $1800 \mathrm{~m}$, 10.04.2005, Uzun 555 (KATO: 17746). Eux. (mt.) el., Endemic, IUCN: LC

403. Cyclamen parviflorum Pobed. subsp. subalpinum (Grey-Wilson) Grey-Wilson

Ormanüstü village, in forest, $1437 \mathrm{~m}$, 12.07.2008, Uzun 1445 (KATO: 17747); ibid., $1479 \mathrm{~m}$, 12.07.2008, Uzun 1446 (KATO: 17748); ibid., 1707 m, 16.07.2008, Uzun 1578 (KATO: 17749); Ormanüstü plateau, in forest, $1615 \mathrm{~m}, 17.07 .2008$, Uzun 1596 (KATO: 17750). Eux. el., Endemic, IUCN: LC

\section{Lysimachia $\mathrm{L}$.}

404. Lysimachia verticillaris Spreng. - Karga otu

Ormanüstü village, grassy place, $950 \mathrm{~m}$, 26.07.2006, Uzun 942 (KATO: 17751); Ormanüstü village, grassy place, $1445 \mathrm{~m}$, 17.07.2008, Uzun 1597 (KATO: 17752). Hyr.-Eux. el.
405. Lysimachia arvensis (L.) U.Manns \& Anderb. subsp. arvensis (syn.: Anagallis arvensis $\mathrm{L}$.)

Köprüyanı district, damp place, $740 \mathrm{~m}$, 05.06.2006, Uzun 902 (KATO: 17753).

406. Lysimachia foemina (Mill.) U.Manns \& Anderb. (syn.: Anagallis foemina Mill.)

Çeşmeler district, stony roadside, $437 \mathrm{~m}$, 07.07.2008, Uzun 1141 (KATO: 17754); Sukenar1 district, damp creekside, $640 \mathrm{~m}$, 07.07.2008, Uzun 1142 (KATO: 17755). Medit. el.

\section{F77 [49]. Ericaceae Juss.}

\section{Rhododendron L. - Orman gülü}

407. Rhododendron luteum Sweet - Sar1 çiçekli orman gülü, Zifin

Ormanüstü village, forest edge, $800 \mathrm{~m}$, 10.05.2005, Uzun 574 (KATO: 17722); Köprüyan1, shrubby place in association with Rhododendron ponticum, 930 m, 23.05.2006, Uzun 868 (KATO: 17723). Eux. el.

408. Rhododendron ponticum L. subsp. ponticum - Mor çiçekli orman gülü, Komar Köprüyan1, in forest, $710 \mathrm{~m}, 23.05 .2006$, Uzun 869 (KATO: 17724). Eux. el.

221. Vaccinium L. - Ayı üzümü

409. Vaccinium myrtillus L. - Çoban üzümü Ormanüstü village, in forest, $1622 \mathrm{~m}$, 15.07.2008, Uzun 1547 (KATO: 17725); Ormanüstü plateau, degraded spruce forest, 1991 m, 02.08.2008, Uzun 1765 (KATO: 17726). Euro-Sib. el.

410. Vaccinium arctostaphylos L. - Trabzon çayı, Likarba, Lifor

Köprüyanı district, rocky place, $860 \mathrm{~m}$, 08.07.2008, Uzun 1282 (KATO: 17727). Eux. el.

\section{Orthilia Rafin.}

411. Orthilia secunda (L.) House Ormanüstü village, pure stand of oriental spruce, $1615 \mathrm{~m}, 26.07 .2006$, Uzun 941 (KATO: 17728); ibid., 1614 m, 10.07.2008, Uzun 1370 (KATO: 17729); Ormanüstü village, in forest, $1622 \mathrm{~m}, 15.07 .2008$, Uzun 1548 (KATO: 17730); Ormanüstü plateau, in forest, $1668 \mathrm{~m}$, 17.07.2008, Uzun 1593 (KATO: 17731); Çamlıdüz plateau, in forest, $1668 \mathrm{~m}$, 17.07.2008, Uzun 1594 (KATO: 17732); Ormanüstü village, in forest, $1710 \mathrm{~m}$, 
21.07.2008, Uzun 1683 (KATO: 17733); ibid., $1752 \mathrm{~m}, 21.07 .2008$, Uzun 1684 (KATO: 17734); Ormanüstü plateau, upper forest border, $2000 \mathrm{~m}, 22.07 .2008$, Uzun 1710 (KATO: 17735).

\section{Pyrola L.}

412. Pyrola media $\mathrm{Sw}$.

Ormanüstü village, in forest, $950 \mathrm{~m}$, 08.07.2005, Uzun 644 (KATO: 17736); Ormanüstü village, in forest, $1622 \mathrm{~m}$, 15.07.2008, Uzun 1549 (KATO: 17737); Ormanüstü plateau, in forest, $1668 \mathrm{~m}$, 17.07.2008, Uzun 1595 (KATO: 17738). Euro-Sib. el.

\section{Monotropa $\mathbf{L}$.}

413. Monotropa hypopitys L.

Köprüyanı district, in forest, $1001 \mathrm{~m}$, 08.07.2008, Uzun 1283 (KATO: 17739).

Parasite

Gentianales Juss. ex Bercht. \& J.Presl

F78 [50]. Rubiaceae Juss.

\section{Crucianella $\mathbf{L}$.}

414. Crucianella gilanica Trin. subsp. pontica (Ehrend.) Ehrend.

Sukenar1 district, roadside slope, $685 \mathrm{~m}$, 11.07.2007, Uzun 1006 (KATO: 18349); Çeşmeler district, rocky place, $910 \mathrm{~m}$, 18.06.2008, Uzun 1111 (KATO: 18350); Çeşmeler district, stony hill, $490 \mathrm{~m}$, 07.07.2008, Uzun 1229 (KATO: 18351); ibid., 634 m, 07.07.2008, Uzun 1230 (KATO: 18352); ibid., 942 m, 08.07.2008, Uzun 1310 (KATO: 18353); Çatak, afforestation site, $873 \mathrm{~m}$, 06.08.2008, Uzun 1833 (KATO: 18354). Eux. el.

\section{Asperula L.}

415. Asperula taurina L. (syn.: Asperula taurina L. subsp. caucasica (Pobed.) Ehrend.)

Köprüyanı district, vicinity of Vazelon monastry, open forest, $1130 \mathrm{~m}, 23.05 .2006$, Uzun 886 (KATO: 18355). Hyr.-Eux. el.

416. Asperula involucrata Wahlenb.

Ormanüstü village, in forest, $1770 \mathrm{~m}$, 08.07.2005, Uzun 719 (KATO: 18356); Köprüyan1 district, in forest, $701 \mathrm{~m}$, 08.07.2008, Uzun 1311 (KATO: 18357); Köprüyanı district, rocky place, $860 \mathrm{~m}$,
08.07.2008, Uzun 1312 (KATO: 18358); ibid., $1017 \mathrm{~m}$, 08.07.2008, Uzun 1313 (KATO: 18359); Köprüyanı district, open forest, $975 \mathrm{~m}$, 08.07.2008, Uzun 1314 (KATO: 18360); Çatak, open forest, $458 \mathrm{~m}$, 14.07.2008, Uzun 1498 (KATO: 18361); Ormanüstü plateau, in forest, $1615 \mathrm{~m}$, 17.07.2008, Uzun 1611 (KATO: 18362). Eux. el.

\section{Galium L. - Yoğurt otu}

417. Galium rotundifolium $\mathrm{L}$.

Ormanüstü village, in forest, $1615 \mathrm{~m}$, 26.07.2006, Uzun 956 (KATO: 18363); Köprüyanı district, in forest, $1001 \mathrm{~m}$, 08.07.2008, Uzun 1315 (KATO: 18364); Çeşmeler district, in forest, $1020 \mathrm{~m}$, 08.07.2008, Uzun 1316 (KATO: 18365). Euro-Sib. el.

418. Galium odoratum (L.) Scop.

Ormanüstü village, in forest, $1680 \mathrm{~m}$, 08.07.2005, Uzun 720 (KATO: 18366).

Euro-Sib. el.

419. Galium verum $\mathrm{L}$. subsp. verum

Çeşmeler district, stony-grassy place, $780 \mathrm{~m}$, 11.07.2007, Uzun 1007 (KATO: 18367); Çeşmeler district, stony hill, $634 \mathrm{~m}$, 07.07.2008, Uzun 1231 (KATO: 18368); Çeşmeler district, stony-grassy place, $890 \mathrm{~m}$, 18.06.2008, Uzun 1112 (KATO: 18369). Euro-Sib. el.

420. Galium fissurense Ehrend \& Schönb.Tem.

Çeşmeler district, stony hill, $1050 \mathrm{~m}$, 11.07.2007, Uzun 1008 (KATO: 18370); Sukenar1 district, stony hill, $663 \mathrm{~m}$, 07.07.2008, Uzun 1232 (KATO: 18372); Köprüyanı district, rocky place, $860 \mathrm{~m}$, 08.07.2008, Uzun 1317 (KATO: 18374); Ormanüstü village, afforestation site, 1683 m, 10.07.2008, Uzun 1385 (KATO: 18376); Çatak, open forest, $458 \mathrm{~m}, 14.07 .2008$, Uzun 1499 (KATO: 18377); Ormanüstü plateau, stony degraded spruce forest, $2010 \mathrm{~m}$, 02.08.2008, Uzun 1789 (KATO: 18378). Eux. el., Endemic, IUCN: LC

421. Galium aparine L.

Çeşmeler district, shrubby place, $444 \mathrm{~m}$, 08.07.2005, Uzun 721 (KATO: 18379); Sukenar1 district, stony hill, $663 \mathrm{~m}$, 07.07.2008, Uzun 1233 (KATO: 18380). Cosmopolitan 
228. Cruciata Mill. - Haç otu

422. Cruciata laevipes Opiz.

Çeşmeler district, grassy places, $880 \mathrm{~m}$, 18.06.2008, Uzun 1113 (KATO: 18381). Euro-Sib. el.

423. Cruciata taurica (Pall. ex Willd.) Ehrend.

Ormanüstü village, grassy place, $1680 \mathrm{~m}$, 08.07.2005, Uzun 722 (KATO: 18382); Ormanüstü plateau, grassy place, $2020 \mathrm{~m}$, 05.08.2005, Uzun 817 (KATO: 18383); Kiremitli district, grassy place, $717 \mathrm{~m}$, 13.07.2007, Uzun 1042 (KATO: 18384). Ir.Tur. el.

\section{F79 [51]. Gentianaceae Juss.}

\section{Blackstonia Hudson}

424. Blackstonia perfoliata (L.) Huds. subsp. perfoliata

Ormanüstü village, open forest edge, $1250 \mathrm{~m}$, 05.08.2005, Uzun 787 (KATO: 18109); Çeşmeler district, roadside, $650 \mathrm{~m}$, 26.07.2006, Uzun 1776 (KATO: 18110); Ormanüstü village, grassy place, $980 \mathrm{~m}$, 26.07.2006, Uzun 1830 (KATO: 18111); Çeşmeler district, degraded spruce forest, $541 \mathrm{~m}$, 06.08.2008, Uzun 585 (KATO: 18112).

\section{Centaurium Hill - Kırmızı kantaron}

425. Centaurium erythraea Rafn subsp. erythraea

Ormanüstü village, grassy place, $980 \mathrm{~m}$, 26.07.2006, Uzun 950 (KATO: 18113). Euro-Sib. el.

\section{Gentiana L. - Centiyan}

426. Gentiana asclepiadea L.

Ormanüstü village, in forest, $1530 \mathrm{~m}$, 05.08.2005, Uzun 788 (KATO: 18114).

Euro-Sib. el.

427. Gentiana cruciata L. subsp. cruciata Çeşmeler district, grassy place, $1600 \mathrm{~m}$, 02.08.2008, Uzun 1776 (KATO: 18115); Çatak, grassy place, $873 \mathrm{~m}, 06.08 .2008$, Uzun 1830 (KATO: 18116). Euro-Sib. el.

428. Gentiana aquatica L.

Ormanüstü plateau, wet meadow, $1800 \mathrm{~m}$, 10.05.2005, Uzun 585 (KATO: 18117).

429. Gentiana pyrenaica L.
Ormanüstü plateau, grassy place, $2000 \mathrm{~m}$, 10.05.2005, Uzun 586 (KATO: 18118). Euro-Sib. el.

430. Gentiana verna L. subsp. pontica (Soltok.) Litard. \& Maire

Ormanüstü plateau, grassy place, $2000 \mathrm{~m}$, 10.05.2005, Uzun 587 (KATO: 18119); Ormanüstü plateau, degraded spruce forest, 1991 m, 02.08.2008, Uzun 1777 (KATO: 18120). Hyr.-Eux. (mt.) el.

Boraginales Juss. ex Bercht. \& J.Presl

F80 [52]. Boraginaceae Juss.

\section{Lappula Fabr.}

431. Lappula barbata (M.Bieb.) Gürke

Sukenar1 district, open place, $498 \mathrm{~m}$, 11.07.2008, Uzun 1427 (KATO: 18135). Ir.Tur. el.

\section{Myosotis L. - Unutmabeni çiçeği}

432. Myosotis arvensis (L.) Hill subsp. arvensis

Ormanüstü village, forest edge, $1745 \mathrm{~m}$, 21.07.2008, Uzun 1691 (KATO: 18136); Çamlıdüz plateau, damp places in forest, 1879 m, 20.07.2008, Uzun 1663 (KATO: 18137). Euro-Sib. el.

433. Myosotis lazica Popov

Ormanüstü plateau, grassy place, $2000 \mathrm{~m}$, 05.06.2006, Uzun 919 (KATO: 18138); Ormanüstü village, forest edge wet hills, $1200 \mathrm{~m}, 10.05 .2005$, Uzun 588 (KATO: 18139). Eux. el., Rare, IUCN: VU

434. Myosotis sylvatica Hoffm. subsp. rivularis Vestergr.

Ormanüstü village, damp hill, $1550 \mathrm{~m}$, 10.05.2005, Uzun 589 (KATO: 18140); Ormanüstü village, roadside, $1380 \mathrm{~m}$, 10.05.2005, Uzun 590 (KATO: 18141). Hyr.Eux. (mt.) el.

435. Myosotis alpestris F.W.Schmidt subsp. alpestris

Ormanüstü plateau, rocky and grassy places, 2000 m, 08.07.2005, Uzun 690 (KATO: 18142).

436. Myosotis olympica Boiss.

Ormanüstü plateau, damp meadows, 1945 m, 08.07.2005, Uzun 691 (KATO: 18143); Çamlıdüz plateau, afforestation site, $1728 \mathrm{~m}$, 20.07.2008, Uzun 1664 (KATO: 18144). Eux. (mt.) el. 
437. Myosotis lithospermifolia (Willd.) Hornem

Ormanüstü plateau, damp meadows, 1945 m, 08.07.2005, Uzun 692 (KATO: 18145).

438. Myosotis sparsiflora Pohl

Çeşmeler district, damp hills, $600 \mathrm{~m}$, 10.05.2005, Uzun 591 (KATO: 18146).

Euro-Sib. el.

\section{Cynoglossum L. - Kedi pençesi}

439. Cynoglossum officinale L. subsp. officinale

Köprüyan1, roadside, $700 \mathrm{~m}, 23.05 .2006$, Uzun 881 (KATO: 18147). Euro-Sib. el.

440. Cynoglossum creticum Mill.

Köprüyan1 district, open place, $900 \mathrm{~m}$, 23.05.2006, Uzun 882 (KATO: 18148); Çeşmeler district, roadside, $750 \mathrm{~m}$, 11.07.2007, Uzun 996 (KATO: 18149).

\section{Lithospermum $\mathbf{L}$.}

441. Lithospermum officinale $\mathrm{L}$.

Çeşmeler district, grassy place, $700 \mathrm{~m}$, 01.06.2008, Uzun 1072 (KATO: 18150); Çeşmeler district, roadside, $890 \mathrm{~m}$, 18.06.2008, Uzun 1105 (KATO: 18151). Euro-Sib. el.

\section{Echium L. - Engerek otu}

442. Echium italicum L. subsp. italicum

Çeşmeler district, roadside, $415 \mathrm{~m}$, 05.08.2005, Uzun 790 (KATO: 18152);

Çeşmeler district, roadside, $1050 \mathrm{~m}$, 11.07.2007, Uzun 997 (KATO: 18153);

Kiremitli district, roadside slopes, 760-900 m, 13.07.2007, Uzun 1039 (KATO: 18154). Medit. el.

443. Echium vulgare L. subsp. vulgare Ormanüstü village, roadside, $1000 \mathrm{~m}$, 10.05.2005, Uzun 592 (KATO: 18155). Euro-Sib. el.

\section{Onosma L. - Emzik otu}

444. Onosma bourgaei Boiss.

Çeşmeler district, meadow ve stony place, $700 \mathrm{~m}, 01.06 .2008$, Uzun 1073 (KATO: 18156). Ir.-Tur. el.

*445. Onosma bornmuelleri Hausskn.

Çeşmeler district, stony hill, $700 \mathrm{~m}$, 01.06.2008, Uzun 1074 (KATO: 18157); Çeşmeler district, stony hill, $583 \mathrm{~m}$, 07.07.2008, Uzun 1198 (KATO: 18158); Yukarıköy village, open place, $931 \mathrm{~m}$,
14.07.2008, Uzun 1487 (KATO: 18159). Ir.Tur. el., Endemic, IUCN: LC

238. Cerinthe L.

446. Cerinthe minor L. subsp. auriculata (Ten.) Domac

Yukarıöy village, open place, $950 \mathrm{~m}$, 14.07.2008, Uzun 1488 (KATO: 18160).

\section{Symphytum L. - Karakafes otu}

447. Symphytum sylvaticum Boiss. subsp. sepulcrale (Boiss. \& Balansa) Greuter \& Burdet

Ormanüstü village, forest edge, $1000 \mathrm{~m}$, 10.05.2005, Uzun 593 (KATO: 18161); Ormanüstü village, forest edge, $1245 \mathrm{~m}$, 13.08.2005, Uzun 857 (KATO: 18162). Eux. el., Endemic, IUCN: NT

\section{Trachystemon D.Don. - Galdirik, Hodan \\ 448. Trachystemon orientalis (L.) G.Don - Tomara \\ Ormanüstü village, damp creekside, $1245 \mathrm{~m}$, 05.06.2006, Uzun 920 (KATO: 18163). Eux. el.}

Solanales Juss. ex Bercht. \& J.Presl

F81 [53]. Convolvulaceae Juss.

241. Convolvulus L.

449. Convolvulus cantabrica L.

Çeşmeler district, grassy place, $1050 \mathrm{~m}$, 11.07.2007, Uzun 995 (KATO: 18129); ibid., 700 m, 01.06.2008, Uzun 1071 (KATO: 18130); Çeşmeler district, stony hill, $490 \mathrm{~m}$, 07.07.2008, Uzun 1197 (KATO: 18131). Medit. el.

450. Convolvulus arvensis L. - Tarla sarmaşı

Çeşmeler district, roadside, $670 \mathrm{~m}$, 05.06.2006, Uzun 918 (KATO: 18132). Cosmopolitan

242. Calystegia R.Br.

451. Calystegia silvatica (Kit.) Griseb. - Çit sarmaşığı

Çeşmeler district, in forest, $529 \mathrm{~m}$, 14.07.2008, Uzun 1486 (KATO: 18133).

243. Cuscuta L.

*452. Cuscuta europaea L. - Bostanbozan 
Vicinity of Vazelon monastry, as climber on Sambucus ebulus, 1200 m, 24.07.2008, Uzun 1741 (KATO: 18134).

F82 [54]. Solanaceae Juss.

244. Solanum L. - İt üzümü

453. Solanum nigrum L. - Siyah meyveli it üzümü

Ceşmeler district, roadside, $650 \mathrm{~m}$, 07.07.2008, Uzun 1195 (KATO: 18121).

Cosmopolitan

454. Solanum dulcamara L.

Ormanüstü village, wet streamside, $900 \mathrm{~m}$, 07.07.2008, Uzun 1196 (KATO: 18122).

Euro-Sib. el.

\section{Alkekengi Mill.}

455. Alkekengi officinarum Moench var. officinarum (bas.: Physalis alkekengi L. var. alkekengi) - Güvey feneri

Köprüyan1 district, roadside slope, $600 \mathrm{~m}$, 23.05.2006, Uzun 880 (KATO: 18123); Ormanüstü village, shrubby place, $1091 \mathrm{~m}$, 05.08.2005, Uzun 789 (KATO: 18124).

\section{Atropa L.}

456. Atropa bella-donna L. - Güzel avrat otu Ormanüstü village, forest edge, $1577 \mathrm{~m}$, 08.07.2005, Uzun 688 (KATO: 18125); Ormanüstü village, roadside, $1450 \mathrm{~m}$, 08.07.2005, Uzun 689 (KATO: 18126). Euro-Sib. el.

247. Datura L.

457. Datura stramonium L. - Boru çiçeği Ormanüstü village, roadside, $780 \mathrm{~m}$, 05.06.2006, Uzun 917 (KATO: 18127). Cosmopolitan

\section{Hyoscyamus $\mathrm{L}$.}

458. Hyoscyamus niger L. - Ban otu

Sukenarı district, roadside slope, $700 \mathrm{~m}$, 01.06.2008, Uzun 1070 (KATO: 18128).

Lamiales Bromhead

F83 [55]. Oleaceae Hoffmanns. \& Link

249. Fraxinus L. - Dışbudak

459. Fraxinus angustifolia Vahl. subsp. oxycarpa (M.Bieb. ex Willd.) Franco \& Rocha Afonso - Sivri meyveli dişbudak
Catak, roadside, $650 \mathrm{~m}, 14.07 .2008$, Uzun 1493 (KATO: 18248). Euro-Sib. el.

250. Ligustrum L. - Kurtbağrı

460. Ligustrum vulgare $\mathrm{L}$.

Çatak, afforestation site, $873 \mathrm{~m}, 06.08 .2008$,

Uzun 1831 (KATO: 18249). Euro-Sib. el.

251. Osmanthus Lour.

461. Osmanthus decorus (Boiss. \& Balansa) Kasapligil

Köprüyan1 district, in forest, $977 \mathrm{~m}$, 11.07.2008, Uzun 1429 (KATO: 18250); Ormanüstü village, in forest, $1205 \mathrm{~m}$, 24.07.2008, Uzun 1744 (KATO: 18251). Eux. el., Rare, IUCN: VU

252. Phillyrea L.

462. Phillyrea latifolia L. - Akçakesme

Çeşmeler, shrubby place, 437 m, 07.07.2008, Uzun 1223 (KATO: 18252); Çatak, open forest, $458 \mathrm{~m}, 14.07 .2008$, Uzun 1494 (KATO: 18253). Medit. el.

F84 [56]. Plantaginaceae Juss.

\section{Plantago L. - Sinir otu}

463. Plantago major L. subsp. major

Köprüyan1, open place, 1200 m, 11.07.2007, Uzun 1003 (KATO: 18246).

464. Plantago lanceolata L.

Ormanüstü village, roadside slope, $1100 \mathrm{~m}$, 05.08.2005, Uzun 803 (KATO: 18247).

254. Linaria Mill.

465. Linaria genistifolia (L.) Mill. subsp. genistifolia

Sindiran village, stony and slopy hill, $800 \mathrm{~m}$, 26.07.2008, Uzun 1751 (KATO: 18266.Euro-Sib. el.

255. Digitalis L. - Yüksük otu

466. Digitalis ferruginea L. subsp. schischkinii (Ivanina) K.Werner - Ar1 kovanı Ormanüstü plateau, open forest, $1700 \mathrm{~m}$, 10.05.2005, Uzun 597 (KATO: 18267); ibid., $1800 \mathrm{~m}, 27.07 .2008$, Uzun 1753 (KATO: 18268). Eux. el.

256. Veronica L. - Yavşan otu

467. Veronica gentianoides Vahl subsp. gentianoides 
Ormanüstü plateau, grassy place, $2000 \mathrm{~m}$, 08.07.2005, Uzun 702 (KATO: 18269); ibid., 2000 m, 05.06.2006, Uzun 924 (KATO: 18270). Hyr.-Eux. (mt.) el.

468. Veronica persica Poiret

Ormanüstü village, open place, $1680 \mathrm{~m}$, 08.07.2005, Uzun 703 (KATO: 18271).

469. Veronica anagallis-aquatica L. subsp. anagallis-aquatica

Ormanüstü village, damp place, $1680 \mathrm{~m}$, 08.07.2005, Uzun 704 (KATO: 18272). Cosmopolitan

470. Veronica beccabunga L. subsp. beccabunga

Ormanüstü village, streamside, $1680 \mathrm{~m}$, 08.07.2005, Uzun 705 (KATO: 18273).

471. Veronica chamaedrys L. subsp. chamaedrys

Çamlıdüz plateau, in forest, $1589 \mathrm{~m}$, 17.07.2008, Uzun 1610 (KATO: 18274); Ormanüstü plateau, afforestation site, 1664 m, 21.07.2008, Uzun 1692 (KATO: 18275). Euro-Sib. el.

472. Veronica multifida L.

Çeşmeler district, grassy place, $700 \mathrm{~m}$, 01.06.2008, Uzun 1077 (KATO: 18276);

Çeşmeler district, stony hill, $583 \mathrm{~m}$, 07.07.2008, Uzun 1224 (KATO: 18277). Ir.-

Tur. el., Endemic, IUCN: LC

473. Veronica peduncularis M.Bieb.

Ormanüstü village, damp hill, $1100 \mathrm{~m}$, 10.05.2005, Uzun 598 (KATO: 18278; Ormanüstü village, open place, $1680 \mathrm{~m}$, 08.07.2005, Uzun 706 (KATO: 18279); Köprüyan1, roadside slope, $1000 \mathrm{~m}$, 23.05.2006, Uzun 885 (KATO: 18280); Ormanüstü plateau, afforestation site, 1862 m, 02.08.2008, Uzun 1782 (KATO: 18281). Eux. el.

474. Veronica officinalis L.

Ormanüstü village, in forest, $1280 \mathrm{~m}$, 09.07.2008, Uzun 1348 (KATO: 18282); ibid., $1710 \mathrm{~m}, 21.07 .2008$, Uzun 1693 (KATO: 18283). Euro-Sib. el.

257. Globularia L.

475. Globularia trichosantha Fisc. \& C.A.Mey. subsp. trichosantha

Çeşmeler district, grassy place, $700 \mathrm{~m}$, 01.06.2008, Uzun 1078 (KATO: 18302).
F85 [57]. Scrophulariaceae Juss.

\section{Scrophularia L. - Siraca otu}

476. Scrophularia scopolii Hoppe ex Pers. var. adenocalyx Sommier \& Levier

Ormanüstü village, in forest, $1140 \mathrm{~m}$, 10.07.2008, Uzun 1381 (KATO: 18264); Çamlıdüz plateau, in forest, $1786 \mathrm{~m}$, 20.07.2008, Uzun 1669 (KATO: 18265). Eux. el.

\section{Verbascum L. - Sığır kuyruğu}

477. Verbascum spectabile M.Bieb. var. spectabile

Ormanüstü plateau, open place, $1750 \mathrm{~m}$, 08.07.2005, Uzun 701 (KATO: 18254); Yukarıköy village, afforestation site, $836 \mathrm{~m}$, 18.07.2008, Uzun 1629 (KATO: 18255); Çamlıdüz plateau, afforestation site, $1728 \mathrm{~m}$, 20.07.2008, Uzun 1667 (KATO: 18256); Ormanüstü plateau, afforestation site, 1862 m, 02.08.2008, Uzun 1781 (KATO: 18257). Eux. el.

478. Verbascum pyramidatum M.Bieb.

Çeşmeler district, stony hill, $890 \mathrm{~m}$, 18.06.2008, Uzun 1109 (KATO: 18258); Ormanüstü village, afforestation site, 1683 m, 10.07.2008, Uzun 1380 (KATO: 18259); Yukarıköy village, open place, $931 \mathrm{~m}$, 14.07.2008, Uzun 1495 (KATO: 18260); Çamlıdüz plateau, afforestation site, $1728 \mathrm{~m}$, 20.07.2008, Uzun 1668 (KATO: 18261). Hyr.-Eux. el.

479. Verbascum thapsus L. subsp. thapsus Köprüyan1 district, pathside in open place, $1862 \mathrm{~m}, 24.07 .2008$, Uzun 1745 (KATO: 18262).Euro-Sib. el.

480. Verbascum gnaphalodes M.Bieb. Çeşmeler district, stony-grassy place, $890 \mathrm{~m}$, 18.06.2008, Uzun 1110 (KATO: 18263). Eux. el.

\section{F86 [58]. Verbenaceae J.St.Hil.}

\section{Verbena L.}

481. Verbena officinalis $\mathrm{L}$.

Sukenar1 district, roadside, $640 \mathrm{~m}$, 07.07.2008, Uzun 1199 (KATO: 18164); Çeşmeler district, roadside, $650 \mathrm{~m}$, 07.07.2008, Uzun 1200 (KATO: 18165). Cosmopolitan 
F87 [59]. Lamiaceae Martinov (= Labiatae Juss.)

\section{Ajuga L. - Mayasıl otu}

482. Ajuga orientalis L.

Ormanüstü plateau, grassy place, $1800 \mathrm{~m}$, 10.05.2005, Uzun 594 (KATO: 18166); ibid., 2000 m, 05.06.2006, Uzun 921 (KATO: 18167).

483. Ajuga reptans L.

Ormanüstü plateau, damp creekside, $1800 \mathrm{~m}$, 05.06.2006, Uzun 922 (KATO: 18168).

Euro-Sib. el.

484. Ajuga chamaepitys (L.) Schreb. subsp. chia (Schreb.) Arcang.

Yukarıköy village, stony hill, $800 \mathrm{~m}$, 14.07.2008, Uzun 1489 (KATO: 18169).

\section{Teucrium L.}

485. Teucrium chamaedrys L. subsp. trapezunticum Rech.f. - Dalak otu

Çeşmeler district, stony hill, $595 \mathrm{~m}$, 08.07.2005, Uzun 693 (KATO: 18170); Karşıyaka village, open hill, $900 \mathrm{~m}$, 13.07.2007, Uzun 1040 (KATO: 18171); Çeşmeler district, stony hill, $437 \mathrm{~m}$, 07.07.2008, Uzun 1201 (KATO: 18172); Çeşmeler district, stony hill, $583 \mathrm{~m}$, 07.07.2008, Uzun 1202 (KATO: 18173); Sukenar1 district, stony hill, $663 \mathrm{~m}$, 07.07.2008, Uzun 1203 (KATO: 18174); Çatak, afforestation site, $477 \mathrm{~m}, 14.07 .2008$, Uzun 1490 (KATO: 18175). Eux. el.

486. Teucrium polium L. subsp. polium Çeşmeler district, stony-grassy place, $890 \mathrm{~m}$, 18.06.2008, Uzun 1106 (KATO: 18176); Köprüyanı, stony place, 900 m, 11.07.2007, Uzun 998 (KATO: 18177); Kiremitli district, grassy place, $717 \mathrm{~m}, 13.07 .2007$, Uzun 1041 (KATO: 18178); Ceşmeler district, stony hill, 437 m, 07.07.2008, Uzun 1204 (KATO: 18179); Çeşmeler district, stony hill, $583 \mathrm{~m}$, 07.07.2008, Uzun 1205 (KATO: 18180); Çeşmeler district, stony hill, $634 \mathrm{~m}$, 07.07.2008, Uzun 1206 (KATO: 18181).

\section{Phlomis L. - Şalba}

487. Phlomis russeliana (Sims) Lag. ex Benth.

Çeşmeler district, forest edge, $717 \mathrm{~m}$, 27.07.2008, Uzun 1725 (KATO: 18182). Eux. (mt.) el., Endemic, IUCN: LC
264. Lamium L. - Ballıbaba

488. Lamium galeobdolon (L.) L. subsp. galeobdolon (syn.: Galeobdolon luteum Hudson subsp. luteum)

Vicinity of Vazelon monastry, in forest, 1069 m, 24.07.2008, Uzun 1743 (KATO: 18187). Euro-Sib. el.

489. Lamium maculatum $\mathrm{L}$.

Ormanüstü village, open forest, $1350 \mathrm{~m}$, 10.05.2005, Uzun 595 (KATO: 18183); Köprüyanı district, forest edge, $1130 \mathrm{~m}$, 23.05.2006, Uzun 883 (KATO: 18184); Vicinity of Vazelon monastry, open forest, 1205 m, 24.07.2008, Uzun 1742 (KATO: 18185). Euro-Sib. el.

490. Lamium moschatum Mill. subsp. micranthum (Boiss.) Mennema (syn.: Lamium ponticum Boiss. \& Balansa ex Boiss.)

Köprüyanı district, rocky roadside slope, 700 m, 23.05.2006, Uzun 884 (KATO: 18186).

Ir.-Tur. el.

\section{Stachys L. - Karabaș otu}

491. Stachys byzantina K.Koch

Çeşmeler district, stony hill, $1050 \mathrm{~m}$, 11.07.2007, Uzun 999 (KATO: 18188); Çeşmeler district, stony place, $700 \mathrm{~m}$, 01.06.2008, Uzun 1075 (KATO: 18189). Euro-Sib. el.

492. Stachys sylvatica $\mathrm{L}$.

Ormanüstü village, damp places in forest, $1100 \mathrm{~m}$, 08.07.2005, Uzun 694 (KATO: 18190). Euro-Sib. el.

493. Stachys iberica M.Bieb. subsp. iberica var. iberica

Çeşmeler district, shrubby place, $750 \mathrm{~m}$, 01.06.2008, Uzun 1076 (KATO: 18191); Çeşmeler district, stony place, $880 \mathrm{~m}$, 18.06.2008, Uzun 1107 (KATO: 18192); Çeşmeler district, stony hill, $490 \mathrm{~m}$, 07.07.2008, Uzun 1207 (KATO: 18193); Çeşmeler district, stony hill, $634 \mathrm{~m}$, 07.07.2008, Uzun 1208 (KATO: 18194). Ir.Tur. el.

494. Stachys аппиа (L.) L. subsp. аппиа (syn.: Stachys annua (L.) L. var. lycaonica R.Bhattacharjee)

Ormanüstü village, roadside slope, $1100 \mathrm{~m}$, 05.08.2005, Uzun 791 (KATO: 18195); Çeşmeler, stony hill, $437 \mathrm{~m}$, 07.07.2008, Uzun 1209 (KATO: 18196). Ir.-Tur. el. 
495. Stachys macrantha (C.Koch) Stearn Ormanüstü plateau, wet meadows, $1815 \mathrm{~m}$, 08.07.2005, Uzun 695 (KATO: 18197); Ormanüstü plateau, grassy place, $1900 \mathrm{~m}$, 05.08.2005, Uzun 792 (KATO: 18198). Eux. el.

266. Prunella L.

496. Prunella vulgaris L. subsp. vulgaris Ormanüstü village, grassy place, $1000 \mathrm{~m}$, 10.05.2005, Uzun 596 (KATO: 18199). Euro-Sib. el.

497. Prunella laciniata (L.) L.

Çeșmeler district, roadside, $910 \mathrm{~m}$, 18.06.2008, Uzun 1108 (KATO: 18200); Çeşmeler district, stony hills, $634 \mathrm{~m}$, 07.07.2008, Uzun 1210 (KATO: 18201). Euro-Sib. el.

\section{Origanum L. - Mercanköşk}

498. Origanum vulgare L. subsp. gracile (K.Koch) Ietsw.

Ormanüstü village, forest edge, $1620 \mathrm{~m}$, 05.08.2005, Uzun 793 (KATO: 18202); Çeşmeler district, stony hill, $490 \mathrm{~m}$, 07.07.2008, Uzun 1211 (KATO: 18203); Sukenar1 district, stony hill, $663 \mathrm{~m}$, 07.07.2008, Uzun 1212 (KATO: 18204); Sukenar1 district, in forest, $498 \mathrm{~m}$, 11.07.2008, Uzun 1428 (KATO: 18205). Ir.Tur. el.

499. Origanum vulgare L. subsp. viridulum (Martrin-Donos) Nyman (syn.: Origanum vulgare L. subsp. viride (Boiss.) Hayek)

Çeşmeler district, stony hill, $440 \mathrm{~m}$, 08.07.2004, Uzun 550, (KATO: 18206); Çeşmeler district, stony hill, $1050 \mathrm{~m}$, 11.07.2007, Uzun 1000 (KATO: 18207).

\section{Satureja L.}

500. Satureja spicigera (K.Koch) Boiss. Köprüyanı district, roadside hill, $780 \mathrm{~m}$, 08.07.2008, Uzun 1305 (KATO: 18208). Eux. el.

\section{Clinopodium $\mathbf{L}$.}

501. Clinopodium grandiflorum (L.) Kuntze (syn.: Calamintha grandiflora (L.) Moench) Ormanüstü plateau, shrubby place, $1814 \mathrm{~m}$, 08.07.2005, Uzun 696 (KATO: 18209); Ormanüstü village, in forest, $1350 \mathrm{~m}$, 26.07.2006, Uzun 951 (KATO: 18210); Çamlıdüz plateau, in forest, $1589 \mathrm{~m}$,
17.07.2008, Uzun 1609 (KATO: 18211). Euro-Sib. el.

502. Clinopodium menthifolium (Host) Merino subsp. menthifolium (syn.: Calamintha sylvatica Bromf. subsp. sylvatica)

Çeşmeler district, stony hills, $437 \mathrm{~m}$, 07.07.2008, Uzun 1213 (KATO: 18212).

Euro-Sib. el.

503. Clinopodium nepeta (L.) Kuntze subsp. nepeta (syn.: Calamintha nepeta (L.) Savi subsp. nepeta)

Çeşmeler, stony hill, 437 m, 07.07.2008, Uzun 1214 (KATO: 18213).

Medit. el.

504. Clinopodium nepeta (L.) Kuntze subsp. spruneri (Boiss.) Bartolucci \& F.Conti (syn.: Calamintha nepeta (L.) Savi subsp. glandulosa (Req.) P.W. Ball)

Çeşmeler district, stony hill, $583 \mathrm{~m}$, 07.07.2008, Uzun 1215 (KATO: 18214); Sukenar1 district, roadside slope, $640 \mathrm{~m}$, 07.07.2008, Uzun 1216 (KATO: 18215).

505. Clinopodium umbrosum (M.Bieb.) K.Koch

Ormanüstü village, forest edge, $1610 \mathrm{~m}$, 05.08.2005, Uzun 796 (KATO: 18224). Hyr.Eux. el.

506. Clinopodium vulgare L. subsp. vulgare Ormanüstü village, forest edge, $1370 \mathrm{~m}$, 08.07.2005, Uzun 697 (KATO: 18216); Çeşmeler district, forest edge, $470 \mathrm{~m}$, 05.08.2005, Uzun 794 (KATO: 18217); Ormanüstü village, forest edge, $1575 \mathrm{~m}$, 05.08.2005, Uzun 795 (KATO: 18218); Sukenar1 district, stony hill, $663 \mathrm{~m}$, 07.07.2008, Uzun 1217 (KATO: 18219); Köprüyanı district, open forest, $975 \mathrm{~m}$, 08.07.2008, Uzun 1306 (KATO: 18220); Catak, afforestation site, $477 \mathrm{~m}, 14.07 .2008$, Uzun 1491 (KATO: 18221); Çamlıdüz plateau, afforestation site, $1728 \mathrm{~m}$, 20.07.2008, Uzun 1665 (KATO: 18222); Ormanüstü plateau, afforestation site, 1862 m, 02.08.2008, Uzun 1778 (KATO: 18223). Euro-Sib. el.

\section{Thymus L. - Kekik}

507. Thymus praecox Opiz subsp. jankae (Čelak.) Jalas (heterotypic syn.: Thymus praecox Opiz subsp. scorpilii (Velen.) Jalas) Ormanüstü village, on rocks, $1680 \mathrm{~m}$, 08.07.2005, Uzun 698 (KATO: 18225); 
Ormanüstü plateau, rocky hills, $1945 \mathrm{~m}$, 08.07.2005, Uzun 699 (KATO: 18226); ibid., 2020 m, 05.08.2005, Uzun 797 (KATO: 18227); ibid., 2000 m, 05.06.2006, Uzun 923 (KATO: 18228); Ormanüstü plateau, degraded spruce forest, $1991 \mathrm{~m}, 02.08 .2008$, Uzun 1779 (KATO: 18229).

508. Thymus nummularius M. Bieb. (syn.: Thymus pseudopulegioides Klokov \& Des.Shost.)

Ormanüstü plateau, grassy place, $2000 \mathrm{~m}$, 05.08.2005, Uzun 798 (KATO: 18230); Çamlıdüz plateau, afforestation site, $1728 \mathrm{~m}$, 20.07.2008, Uzun 1666 (KATO: 18231); Ormanüstü plateau, degraded spruce forest, 1991 m, 02.08.2008, Uzun 1780 (KATO: 18232).

\section{Mentha L. - Nane}

509. Mentha longifolia (L.) L. subsp. longifolia

Ormanüstü village, damp place, $1750 \mathrm{~m}$, 05.08.2005, Uzun 799 (KATO: 18233); Sukenar1 district, wet open place, $640 \mathrm{~m}$, 07.07.2008, Uzun 1218 (KATO: 18234). Eux. el.

\section{Salvia L. - Adaçayı}

510. Salvia forskohlei L.

Ormanüstü village, in forest, $974 \mathrm{~m}$, 05.08.2005, Uzun 800 (KATO: 18235). Eux. el.

511. Salvia glutinosa $\mathrm{L}$.

Ormanüstü village, in forest, $974 \mathrm{~m}$, 05.08.2005, Uzun 801 (KATO: 18236).

Hyr.-Eux. el.

512. Salvia virgata Jacq.

Çeşmeler district, grassy place, $458 \mathrm{~m}$, 05.08.2005, Uzun 802 (KATO: 18237); Catak district, roadside slope, $600 \mathrm{~m}$, 11.07.2007, Uzun 1001 (KATO: 18238); Sukenar1 district, open place, $640 \mathrm{~m}$, 07.07.2008, Uzun 1219 (KATO: 18239). Ir.Tur. el.

513. Salvia verticillata $\mathrm{L}$. subsp. verticillata Çeşmeler district, grassy place, $440 \mathrm{~m}$, 08.07.2005, Uzun 700 (KATO: 18240). Euro-Sib. el.

514. Salvia verticillata L. subsp. amasiaca (Freyn \& Bornm.) Bornm.

Köprüyan1 district, forest edge, $1200 \mathrm{~m}$, 11.07.2007, Uzun 1002 (KATO: 18241); Çeşmeler district, stony hills, $490 \mathrm{~m}$,
07.07.2008, Uzun 1220 (KATO: 18242); Çeşmeler district, open place, $583 \mathrm{~m}$, 07.07.2008, Uzun 1221 (KATO: 18243); ibid., 634 m, 07.07.2008, Uzun 1492 (KATO: 18244); Yukarıköy village, open place, 931 m, 14.07.2008, Uzun 1003 (KATO: 18245). Ir.-Tur. el.

\section{F88 [60]. Orobanchaceae Vent.}

\section{Orobanche L. - Canavar out}

515. Orobanche alba Stephan ex Willd.

Ormanüstü village, forest edge, $929 \mathrm{~m}$, 08.07.2005, Uzun 710 (KATO: 18304); Çamlıdüz plateau, afforestation site, $1728 \mathrm{~m}$, 20.07.2008, Uzun 1670 (KATO: 18305). Parasite

516. Orobanche caryophyllacea $\mathrm{Sm}$.

Ormanüstü village, roadside slope, $1600 \mathrm{~m}$, 16.07.2008, Uzun 1582 (KATO: 18306). Parasite

\section{Phelipanche Pomel}

517. Phelipanche ramosa (L.) Pomel (syn.: Orobanche ramosa L.)

Çeşmeler district, stony hill, $583 \mathrm{~m}$, 07.07.2008, Uzun 1226 (KATO: 18303). Parasite

\section{Melampyrum L.}

518. Melampyrum arvense L. var. elatius Boiss.

Çeşmeler district, stony hill, $850 \mathrm{~m}$, 11.07.2007, Uzun 1004 (KATO: 18284); Çeşmeler district, stony hill, $583 \mathrm{~m}$, 07.07.2008, Uzun 1225 (KATO: 18285); Köprüyanı district, rocky place, $1017 \mathrm{~m}$, 08.07.2008, Uzun 1307 (KATO: 18286); Köprüyan1 district, in forest, $964 \mathrm{~m}$, 08.07.2008, Uzun 1308 (KATO: 18287); Çeşmeler district, degraded spruce forest, 541 m, 06.08.2008, Uzun 1832 (KATO: 18288). Eux. el., Endemic, IUCN: NT

\section{Euphrasia L. - Gözlük otu, Göz otu}

519. Euphrasia officinalis L. subsp. rostkoviana (Hayne) Towns (syn.: Euphrasia rostkoviana Hayne subsp. rostkoviana)

Ormanüstü plateau, grassy place, $1850 \mathrm{~m}$, 05.05.2005, Uzun 556 (KATO: 18289); Ormanüstü plateau, grassy place, $1900 \mathrm{~m}$, 08.07.2005, Uzun 707 (KATO: 18290). Euro-Sib. el. 
520. Euphrasia stricta J.P.Wolff ex J.F.Lehm. var. pectinata (Ten.) O.Bolòs \& Vigo (bas.: Euphrasia pectinata Ten.)

Ormanüstü village, forest edge, $929 \mathrm{~m}$, 08.07.2005, Uzun 708 (KATO: 18291); Ormanüstü plateau, grassy place, $1850 \mathrm{~m}$, 05.08.2005, Uzun 804 (KATO: 18292); Ormanüstü village, grassy place, $1470 \mathrm{~m}$, 12.07.2008, Uzun 1450 (KATO: 18293); Ormanüstü plateau, afforestation site, 1862 m, 02.08.2008, Uzun 1783 (KATO: 18294). Euro-Sib. el.

521. Euphrasia petiolaris Wettst.

Ormanüstü plateau, grassy place, $1850 \mathrm{~m}$, 05.08.2005, Uzun 805 (KATO: 18295); ibid., $1900 \mathrm{~m}$, 05.08.2005, Uzun 806 (KATO: 18296). Eux. (mt.) el.

\section{Pedicularis L.}

522. Pedicularis condensata M.Bieb.

Ormanüstü plateau, afforestation site, 1862 m, 02.08.2008, Uzun 1784 (KATO: 18297).

Eux. (mt.) el.

\section{Rhinanthus L.}

523. Rhinanthus angustifolius C.C.Gmel. subsp. grandiflorus (Wallr.) D.A.Webb Ormanüstü plateau, grassy place, $2000 \mathrm{~m}$, 08.07.2005, Uzun 709 (KATO: 18298); Ormanüstü plateau, wet meadows, $2020 \mathrm{~m}$, 05.08.2005, Uzun 807 (KATO: 18299).

\section{Rhynchocorys Griseb.}

524. Rhynchocorys elephas (L.) Griseb. subsp. elephas

Ormanüstü village, in forest, $1085 \mathrm{~m}$, 10.07.2008, Uzun 1382 (KATO: 18300). Euro-Sib. el.

525. Rhynchocorys stricta (K.Koch) Albov Ormanüstü village, forest edge, $1670 \mathrm{~m}$, 05.08.2005, Uzun 808 (KATO: 18301). Eux. el.

\section{Aquifoliales Senft}

\section{F89 [61]. Aquifoliaceae Bercht. \& J.Presl}

\section{Ilex L. - Çoban püskülü}

526. Ilex colchica Pojark. subsp. colchica Işığan, Işı1lgan

Ormanüstü village, in forest, $1046 \mathrm{~m}$, 20.07.2008, Uzun 1652 (KATO: 17984). Eux. el.
Asterales Link

F90 [62]. Campanulaceae Juss.

\section{Campanula L. - Çan çiçeği}

527. Campanula latifolia $\mathrm{L}$.

Ormanüstü village, open forest, $929 \mathrm{~m}$, 08.07.2005, Uzun 711 (KATO: 18307); ibid., 1200 m, 05.08.2005, Uzun 809 (KATO: 18308); Ormanüstü village, forest edge, 1670 m, 05.08.2005, Uzun 810 (KATO: 18309); Köprüyanı district, in forest, $1001 \mathrm{~m}$, 08.07.2008, Uzun 1309 (KATO: 18310); Around Vazelon monastery, open forest, 1205 m, 24.07.2008, Uzun 1746 (KATO: 18311); Ormanüstü village, forest edge, 1350 m, 26.07.2006, Uzun 952 (KATO: 18312). Euro-Sib. el.

528. Campanula rapunculoides L. subsp. rapunculoides

Ormanüstü village, in forest, $1680 \mathrm{~m}$, 08.07.2005, Uzun 712 (KATO: 18313); ibid., open forest, $1570 \mathrm{~m}, 05.08 .2005$, Uzun 811 (KATO: 18314); Çeşmeler district, stony hill, 583 m, 07.07.2008, Uzun 1227 (KATO: 18315); Sukenar1 district, stony hill, $663 \mathrm{~m}$, 07.07.2008, Uzun 1228 (KATO: 18316); Çatak, in forest, $483 \mathrm{~m}, 14.07 .2008$, Uzun 1496 (KATO: 18317); Çatak, in forest, 1010 m, 14.07.2008, Uzun 1497 (KATO: 18318). Euro-Sib. el.

529. Campanula rapunculoides L. subsp. cordifolia (K.Koch) Damboldt

Çeşmeler district, forest edge, $700 \mathrm{~m}$, 26.07.2006, Uzun 953 (KATO: 18319); Ormanüstü plateau, afforestation site, 1664 m, 21.07.2008, Uzun 1694 (KATO: 18320).

530. Campanula glomerata L. (syn.: Campanula glomerata L. subsp. hispida (Witasek) Hayek)

Ormanüstü village, forest edge, $1350 \mathrm{~m}$, 08.07.2005, Uzun 713 (KATO: 18321). Euro-Sib. el.

531. Campanula alliariifolia Willd. Ormanüstü village, forest edge, $1300 \mathrm{~m}$, 08.07.2005, Uzun 714 (KATO: 18322); Çeşmeler district, forest edge, $458 \mathrm{~m}$, 05.08.2005, Uzun 812 (KATO: 18323); Köprüyan1, forest edge, 900 m, 11.07.2007, Uzun 1005 (KATO: 18324). Eux. el.

532. Campanula collina Sims subsp. collina Ormanüstü village, afforestation site, 1683 m, 10.07.2008, Uzun 1383 (KATO: 18325); Çamlıdüz plateau, in forest, $1769 \mathrm{~m}$, 
22.07.2008, Uzun 1717 (KATO: 18326); Ormanüstü plateau, degraded spruce forest, 1991 m, 02.08.2008, Uzun 1785 (KATO: 18327); ibid., $2000 \mathrm{~m}, 02.08 .2008$, Uzun 1786 (KATO: 18328); ibid., $2010 \mathrm{~m}$, 02.08.2008, Uzun 1787 (KATO: 18329). Eux. el.

533. Campanula betulifolia K.Koch var. betulifolia

Catak, rock cracks, 400 m, 11.07.2008, Uzun 1430 (KATO: 18330). Eux. el., Endemic, IUCN: LC

534. Campanula tridentata Schreb.

Ormanüstü plateau, on rocks, $2000 \mathrm{~m}$, 08.07.2005, Uzun 715 (KATO: 18331). Eux. (mt.) el.

535. Campanula aucheri A.DC.

Ormanüstü plateau, on rocks, $2000 \mathrm{~m}$, 08.07.2005, Uzun 716 (KATO: 18332); Ormanüstü plateau, stony place, $2000 \mathrm{~m}$, 05.06.2006, Uzun 925 (KATO: 18333). Eux. (mt.) el.

536. Campanula lactiflora M.Bieb.

Yenimahalle, forest edge, $780 \mathrm{~m}, 26.07 .2006$, Uzun 954 (KATO: 18334). Eux. el.

537. Campanula stevenii M.Bieb. subsp. stevenii

Ormanüstü plateau, stony-grassy place, 2000 m, 05.06.2006, Uzun 926 (KATO: 18335). Hyr.-Eux. el.

538. Campanula stevenii M.Bieb. subsp. beauverdiana (Fomin) Rech.f. \& SchimanCzeika

Ormanüstü plateau, grassy place, $1850 \mathrm{~m}$, 05.08.2005, Uzun 813 (KATO: 18336); Ormanüstü plateau, open forest, $1800 \mathrm{~m}$, 27.07.2008, Uzun 814 (KATO: 18337); Ormanüstü plateau, degraded spruce forest, $1991 \mathrm{~m}, 02.08 .2008$, Uzun 815 (KATO: 18338). Ir.-Tur. el.

539. Campanula olympica Boiss.

Ormanüstü village, open forest, $929 \mathrm{~m}$, 08.07.2005, Uzun 717 (KATO: 18339); Ormanüstü plateau, grassy place, $1815 \mathrm{~m}$, 08.07.2005, Uzun 718 (KATO: 18340); Ormanüstü village, grassy place, $1220 \mathrm{~m}$, 05.08.2005, Uzun 816 (KATO: 18341); ibid., $1162 \mathrm{~m}$, 09.07.2008, Uzun 1349 (KATO: 18342); Ormanüstü village, afforestation site, 1683 m, 10.07.2008, Uzun 1384 (KATO: 18343); Çamlıdüz plateau, afforestation site, 1728 m, 20.07.2008, Uzun 1671 (KATO: 18344); Ormanüstü plateau, afforestation site, $1862 \mathrm{~m}, \quad 02.08 .2008$, Uzun 1788 (KATO: 18345). Eux. el.

540. Campanula rapunculus $\mathrm{L}$. var. lambertiana (A.DC.) Rech.f.

Yenimahalle, roadside slope, $740 \mathrm{~m}$, 26.07.2006, Uzun 955 (KATO: 18346); Çamlıdüz plateau, grassy place, $1740 \mathrm{~m}$, 22.07.2008, Uzun 1718 (KATO: 18347); Çamlıdüz plateau, open forest, $1892 \mathrm{~m}$, 20.07.2008, Uzun 1672 (KATO: 18348). Euro-Sib. el.

F91[63]. Asteraceae Bercht. \& J.Presl (= Compositae Giseke)

\section{Telekia Baumg.}

541. Telekia speciosa (Schreb.) Baumg.

Ormanüstü village, in forest, $1091 \mathrm{~m}$, 05.08.2005, Uzun 823 (KATO: 18418).

Euro-Sib. el.

\section{Pentanema Cass. - Andiz otu}

542. Pentanema squarrosum (L.) D. Gut. Larr., Santos-Vicente, Anderb., E.Rico \& M.M.Mart.Ort (syn.: Inula conyzae (Griess.) Meikle)

Ormanüstü village, stony open hills, $1050 \mathrm{~m}$, 05.08.2005, Uzun 824 (KATO: 18419); Çatak, in forest, $483 \mathrm{~m}, 14.07 .2008$, Uzun 1502 (KATO: 18420); Çeşmeler district, in forest, $529 \mathrm{~m}$, 14.07.2008, Uzun 1503 (KATO: 18421). Euro-Sib. el.

\section{Pulicaria Gaertner}

543. Pulicaria dysenterica (L.) Bernh. subsp. dysenterica

Cami district, roadside, $600 \mathrm{~m}, 28.07 .2008$, Uzun 1755 (KATO: 18422).

\section{Antennaria Gaertner}

544. Antennaria dioica (L.) Gaertn.

Ormanüstü plateau, stony grassy place, 1900 m, 08.07.2005, Uzun 729 (KATO: 18423); Ormanüstü plateau, stony grassy place, 2014 m, 08.07.2005, Uzun 730 (KATO: 18424); ibid., 2015 m, 05.08.2005, Uzun 825 (KATO: 18425). Euro-Sib. el.

\section{Omalotheca Cass.}

545. Omalotheca sylvatica (L.) Sch. Bip. \& F.W.Schultz (syn.: Gnaphalium sylvaticum L.) 
Ormanüstü plateau, afforestation site, 1862 m, 02.08.2008, Uzun 1791 (KATO: 18426). Euro-Sib. el.

\section{Bombycilaena (DC.) Smolj}

*546. Bombycilaena erecta (L.) Smoljan.

Çeşmeler district, stony-grassy places, 700 m, 17.07.2008, Uzun 1612 (KATO: 18427).

\section{Solidago L. - Altın başak}

547. Solidago virgaurea L. subsp. minuta (L.) Arcang. (syn.: Solidago virgaurea L. subsp. alpestris (Waldst. \& Kit.) Gaudin) Ormanüstü village, open place, $1577 \mathrm{~m}$, 08.07.2005, Uzun 731 (KATO: 18428). Euro-Sib. el.

289. Kemulariella Tamamsch.

548. Kemulariella caucasica (Willd.) Tamamsch. (syn.: Aster caucasicus Willd.) Çamlıdüz plateau, forest edge, $1769 \mathrm{~m}$, 22.07.2008, Uzun 1719 (KATO: 18429). Eux. el.

\section{Erigeron L.}

549. Erigeron annuus (L.) Desf.

Çatak, roadside, $500 \mathrm{~m}, 08.07 .2005$, Uzun 732 (KATO: 18430). Exotic

550. Erigeron canadensis L. (syn.: Conyza canadensis (L.) Cronquist)

Çatak, roadside, $500 \mathrm{~m}$, 08.07.2005, Uzun 733 (KATO: 18431). Exotic

\section{Bellis L.}

551. Bellis perennis L. - Koyungözü papatya Ormanüstü village, open place, $1577 \mathrm{~m}$, 08.07.2005, Uzun 734 (KATO: 18432).

Euro-Sib. el.

\section{Senecio L. - Kanarya otu}

552. Senecio nemorensis L. subsp. nemorensis

Ormanüstü village, inside damp forest, 1091 m, 05.08.2005, Uzun 826 (KATO: 18433).

553. Senecio vulgaris L. subsp. vulgaris Çatak, roadside, $400 \mathrm{~m}, 05.08 .2005$, Uzun 827 (KATO: 18434); Ormanüstü village, wet roadside, $850 \mathrm{~m}$, 26.07.2006, Uzun 960 (KATO: 18435); Sukenarı district, wet open place, $640 \mathrm{~m}, 07.07 .2008$, Uzun 1236 (KATO: 18436).

\section{Tussilago L.}

554. Tussilago farfara L. - Öksürük otu Ormanüstü village, roadside, $1550 \mathrm{~m}$, 10.05.2005, Uzun 599 (KATO: 18437).EuroSib. el.

\section{Petasites Mill.}

555. Petasites albus (L.) P.Gaertn. - Kabalak Ormanüstü village, roadside, $1250 \mathrm{~m}$, 11.07.2007, Uzun 1010 (KATO: 18438). Euro-Sib. el.

\section{Eupatorium L.}

556. Eupatorium cannabinum L. subsp. cannabinum - Sitma otu

Çeşmeler district, damp hill, $700 \mathrm{~m}$, 05.08.2005, Uzun 828 (KATO: 18439); Çeşmeler district, roadside, $470 \mathrm{~m}$, 05.08.2005, Uzun 829 (KATO: 18440). Euro-Sib. el.

\section{Cota J.Gay - Papatya}

557. Cota tinctoria (L.) J.Gay var. pallida (DC.) Özbek \& Vural (syn.: Anthemis tinctoria $\mathrm{L}$. var. pallida DC.)

Çeşmeler district, stony slope, $1050 \mathrm{~m}$, 11.07.2007, Uzun 1011 (KATO: 18441); Köprüyanı district, rocky place, $860 \mathrm{~m}$, 08.07.2008, Uzun 1318 (KATO: 18442); ibid., 1017 m, 08.07.2008, Uzun 1319 (KATO: 18443); Ormanüstü village, afforestation site, $1683 \mathrm{~m}, 10.07 .2008$, Uzun 1387 (KATO: 18444); Çatak, roadside, 873 m, 18.07.2008, Uzun 1630 (KATO: 18445). 558. Cota tinctoria (L.) J.Gay var. tinctoria (syn.: Anthemis tinctoria L. var. tinctoria) Çeşmeler district, stony slope, $1050 \mathrm{~m}$, 11.07.2007, Uzun 1012 (KATO: 18446); Çeşmeler, stony roadside, $437 \mathrm{~m}$, 07.07.2008, Uzun 1237 (KATO: 18447); Çatak, afforestation site, $477 \mathrm{~m}, 14.07 .2008$, Uzun 1504 (KATO: 18448); Çatak, roadside, 873 m, 18.07.2008, Uzun 1631 (KATO: 18449).

\section{Achillea L. - Civanperçemi}

559. Achillea biserrata M.Bieb.

Köprüyan1 district, open forest, $975 \mathrm{~m}$, 08.07.2008, Uzun 1320 (KATO: 18450). Eux. el. 
*560. Achillea pannonica Scheele (syn.: Achillea millefolium L. subsp. pannonica (Scheele) Hayek)

Kiremitli district, grassy place, 760-900 m, 13.07.2007, Uzun 1044 (KATO: 18451); Çatak, afforestation site, $873 \mathrm{~m}, 06.08 .2008$, Uzun 1834 (KATO: 18452). Euro-Sib. el.

561. Achillea setacea Waldst. \& Kit.

Çeşmeler district, stony grassy place, 1050 m, 11.07.2007, Uzun 1013 (KATO: 18453). Euro-Sib. el.

\section{Leucanthemum Mill}

562. Leucanthemum vulgare (Vaill.) Lam. subsp. vulgare

Ormanüstü village, grassy place, $929 \mathrm{~m}$, 08.07.2005, Uzun 735 (KATO: 18454); ibid., $1680 \mathrm{~m}, 08.07 .2005$, Uzun 736 (KATO: 18455); Çeşmeler district, stony slope, 1050 m, 11.07.2007, Uzun 1014 (KATO: 18456); Ormanüstü village, grassy place, $1162 \mathrm{~m}$, 09.07.2008, Uzun 1352 (KATO: 18457); Ormanüstü village, open forest, $1086 \mathrm{~m}$, 10.07.2008, Uzun 1388 (KATO: 18458); Ormanüstü plateau, grassy place, $1800 \mathrm{~m}$, 18.07.2008, Uzun 1632 (KATO: 18459). Euro-Sib. el.

\section{Tanacetum L.}

563. Tanacetum parthenium (L.) Sch.Bip. Gümüş düğme

Ormanüstü village, roadside, $1680 \mathrm{~m}$, 08.07.2005, Uzun 737 (KATO: 18460); Çeşmeler district, roadside, $1050 \mathrm{~m}$, 11.07.2007, Uzun 1015 (KATO: 18461); Ormanüstü village, in forest, $1475 \mathrm{~m}$, 21.07.2008, Uzun 1695 (KATO: 18462). Cosmopolitan

\section{Tripleurospermum Schultz Bip.}

564. Tripleurospermum oreades (Boiss.) Rech.f. var. oreades

Köprüyan1, grassy place, 600 m, 23.05.2006, Uzun 888 (KATO: 18463).

565. Tripleurospermum elongatum (DC.) Bornm.

Köprüyanı, open place, 437 m, 11.07.2007, Uzun 1016 (KATO: 18464).

\section{Arctium L. - Galabak}

566. Arctium minus (Hill) Bernh. - Galabak Çeşmeler district, roadside, $750 \mathrm{~m}$, 26.07.2006, Uzun 961 (KATO: 18465).
302. Cirsium Mill. - Köygöçüren

567. Cirsium trachylepis Boiss.

Ormanüstü plateau, afforestation site, 1664 m, 21.07.2008, Uzun 1696 (KATO: 18466).

Eux. el., Endemic, IUCN: NT

568. Cirsium sommieri Petrak

Çatak, roadside, hill, $550 \mathrm{~m}, 01.06 .2008$, Uzun 1079 (KATO: 18467). Ir.-Tur. el., Endemic, IUCN: LC

569. Cirsium vulgare (Savi) Ten

Çeşmeler district, roadside, $850 \mathrm{~m}$, 26.07.2006, Uzun 962 (KATO: 18468).

570. Cirsium hypoleucum DC.

Çeşmeler district, forest edge, shady place, $550 \mathrm{~m}, 01.06 .2008$, Uzun 1080 (KATO: 18469). Eux. el.

571. Cirsium arvense (L.) Scop. (syn.: Cirsium arvense (L.) Scop. subsp. vestitum (Wimmer \& Grab) Petrak)

Kiremitli, roadside, 760-900 m, 13.07.2007, Uzun 1045 (KATO: 18470).

\section{Picnomon Adans.}

572. Picnomon acarna (L.) Cass.

Çeşmeler district, stony roadside, $437 \mathrm{~m}$, 07.07.2008, Uzun 1238 (KATO: 18471). Medit. el.

\section{Carduus L.}

573. Carduus nutans L. subsp. nutans - Deve dikeni

Çeşmeler district, stony-grassy place, $700 \mathrm{~m}$, 01.06.2008, Uzun 1081 (KATO: 18472).

574. Carduus adpressus C.A.Mey.

Ormanüstü village, roadside, $1445 \mathrm{~m}$, 17.07.2008, Uzun 1613 (KATO: 18473). Eux. el.

575. Carduus pycnocephalus L. subsp. albidus (M.Bieb.) Kazmi

Çeşmeler district, stony place, $700 \mathrm{~m}$, 01.06.2008, Uzun 1082 (KATO: 18474).

\section{Jurinea Cass.}

576. Jurinea consanguinea DC.

Çeşmeler district, stony shrubby place, 700 m, 01.06.2008, Uzun 1083 (KATO: 18475); Çeşmeler district, stony place, $880 \mathrm{~m}$, 18.06.2008, Uzun 1115 (KATO: 18476); Çeşmeler district, stony hill, $490 \mathrm{~m}$, 07.07.2008, Uzun 1239 (KATO: 18477); Çeşmeler district, stony hill, $583 \mathrm{~m}$, 07.07.2008, Uzun 1240 (KATO: 18478); 
Çeşmeler district, stony hill, $634 \mathrm{~m}$, 07.07.2008, Uzun 1241 (KATO: 18479).

577. Jurinea mollis (L.) Rchb.

Çeşmeler district, stony hill, $560 \mathrm{~m}$, 07.07.2008, Uzun 1242 (KATO: 18480).

\section{Centaurea L. - Peygamber çiçeği}

578. Centaurea jacea L. subsp. jacea

Ormanüstü village, grassy place, $1100 \mathrm{~m}$, 05.08.2005, Uzun 830 (KATO: 18481); Çeşmeler district, grassy place, $750 \mathrm{~m}$, 12.07.2007, Uzun 1026 (KATO: 18482).

Euro-Sib. el.

579. Centaurea helenioides Boiss.

Ormanüstü village, forest edge, $1445 \mathrm{~m}$, 17.07.2008, Uzun 1614 (KATO: 18483).

Eux. el., Endemic, IUCN: NT

580. Centaurea iberica Trevir. ex Spreng.

Maçka-Çatak, Hamsiköy aspect, roadside, $601 \mathrm{~m}, 29.07 .2008$, Uzun 1757 (KATO): 18484.

581. Centaurea urvillei DC. subsp. stepposa Wagenitz

Çeşmeler district, stony hill, 1200, 13.07.2007, Uzun 1046 (KATO: 18485); Çeşmeler district, stony place, $700 \mathrm{~m}$, 01.06.2008, Uzun 1084 (KATO: 18486). Ir.-

Tur. el., Endemic, IUCN: LC

307. Psephellus Cass. - Peygamber çiçeği

582. Psephellus hypoleucus (DC.) Boiss. (syn.: Centaurea hypoleuca DC.)

Köprüyanı district, slope, 600 m, 23.05.2006, Uzun 889 (KATO: 18487); Çeşmeler district, stony hill, $700 \mathrm{~m}, 01.06 .2008$, Uzun 1085 (KATO: 18488). Eux. el.

308. Carthamus L. - Aspir

583. Carthamus lanatus $\mathrm{L}$.

Köprüyan1, open place, 980 m, 11.07.2007, Uzun 1017 (KATO: 18489).

309. Carlina $\mathbf{L}$.

584. Carlina vulgaris L. subsp. vulgaris

Çeşmeler district, roadside, $780 \mathrm{~m}$, 11.07.2007, Uzun 1018 (KATO: 18490).

\section{Echinops L. - Topuz}

585. Echinops ossicus K.Koch (syn.: Echinops galaticus Freyn.)

Çeşmeler district, roadside, $1050 \mathrm{~m}$, 11.07.2007, Uzun 1019 (KATO: 18491). Eux. el.
311. Cichorium L.

586. Cichorium intybus L. - Hindiba

Çeşmeler district, stony hill, $437 \mathrm{~m}$, 07.07.2008, Uzun 1243 (KATO: 18492).

Cosmopolitan

\section{Tragopogon $\mathrm{L}$.}

587. Tragopogon porrifolius L. subsp. longirostris (Sch.Bip.) Greuter (bas.: Tragopogon longirostris Bischoff ex Sch. Bip.)

Çeşmeler district, Carpinus orientalis thicket, $750 \mathrm{~m}, 01.06 .2008$, Uzun 1086 (KATO: 18493).

\section{Leontodon L. - Aslandişi}

588. Leontodon hispidus L. subsp. hispidus Ormanüstü plateau, open place, $1800 \mathrm{~m}$, 05.08.2005, Uzun 831 (KATO: 18494); Ormanüstü plateau, afforestation site, 1862 m, 02.08.2008, Uzun 1792 (KATO: 18495).

589. Leontodon hispidus L. subsp. hastilis (L.) Corb. (des. inval.: Leontodon hispidus L. subsp. glabratus (W.Koch) Bisch.)

Ormanüstü plateau, grassy place, $1945 \mathrm{~m}$, 08.07.2005, Uzun 738 (KATO: 18496); Ormanüstü village, open place, $1300 \mathrm{~m}$, 05.08.2005, Uzun 832 (KATO: 18497); Ormanüstü plateau, grassy place, $2020 \mathrm{~m}$, 05.06.2006, Uzun 928 (KATO: 18498); Çeşmeler district, degraded spruce forest, $541 \mathrm{~m}, 06.08 .2008$, Uzun 1835 (KATO: 18499). Euro-Sib. el.

*590. Leontodon oxylepis Boiss. \& Heldr.

Çeşmeler district, stony hills, $634 \mathrm{~m}$, 07.07.2008, Uzun 1244 (KATO: 18500); Çeşmeler district, stony hill, $942 \mathrm{~m}$, 08.07.2008, Uzun 1321 (KATO: 18501); Çeşmeler district, stony hill, $632 \mathrm{~m}$, 11.07.2008, Uzun 1433 (KATO: 18502); Çatak, open forest, $458 \mathrm{~m}, 14.07 .2008$, Uzun 1505 (KATO: 18503). Medit. (mt.) el.

\section{Picris L.}

591. Picris hieracioides L. subsp. hieracioides

Çeşmeler district, stony roadside, $1050 \mathrm{~m}$, 11.07.2007, Uzun 1020 (KATO: 18504); Çatak, in forest, $483 \mathrm{~m}, 14.07 .2008$, Uzun 1506 (KATO: 18505). Euro-Sib. el. 
315. Sonchus L.

592. Sonchus oleraceus L.

Ormanüstü village, open place, $1200 \mathrm{~m}$, 08.07.2005, Uzun 739 (KATO: 18506).

\section{Reichardia Roth.}

593. Reichardia dichotoma (DC.) Freyn (homotypic syn.: Reichardia glauca V.A.Matthews)

Köprüyan1, open place, 700 m, 11.07.2007, Uzun 1021 (KATO: 18507); Çeşmeler district, stony hill, $490 \mathrm{~m}, 07.07 .2008$, Uzun 1245 (KATO: 18508). Ir.-Tur. el.

\section{Hieracium L. - Şahin otu \\ 594. Hieracium subsilvularum (Zahn) P.D.Sell \& C.West \\ Ormanüstü village, in forest, $1710 \mathrm{~m}$, 21.07.2008, Uzun 1697 (KATO: 18509).} Eux. el., Endemic, IUCN: DD

595. Hieracium gentiliforme (Zahn) P.D.Sell $\&$ C.West

Ormanüstü plateau, pure Picea orientalis stand, $1614 \mathrm{~m}$, 10.07.2008, Uzun 1389 (KATO: 18510); Yukarıöy village, in forest, $931 \mathrm{~m}$, 14.07.2008, Uzun 1507 (KATO: 18511); Çamlıdüz plateau, pure Picea orientalis stand, $1892 \mathrm{~m}, 20.07 .2008$, Uzun 1673 (KATO: 18512). Eux. el., Endemic, IUCN: VU

596. Hieracium medianiforme (Litv. \& Zahn) Üksip

Ormanüstü village, in forest, $1647 \mathrm{~m}$, 15.07.2008, Uzun 1558 (KATO: 18513); Çamlıdüz plateau, in forest, $1740 \mathrm{~m}$, 22.07.2008, Uzun 1720 (KATO: 18514). Eux. el.

597. Hieracium transiens (Freyn) Freyn subsp. karagoellense (Zahn) Greuter (syn.: Hieracium karagoellense (Zahn) P.D.Sell \& C.West)

Ormanüstü plateau, afforestation site, 1862 m, 02.08.2008, Uzun 1793 (KATO: 18515). Eux. el., Endemic, IUCN: LC

598. Hieracium transiens (Freyn) Freyn subsp. erythrocarpum (Peter) Greuter (syn.: Hieracium erythrocarpum Peter)

Ormanüstü village, in forest, $1615 \mathrm{~m}$, 26.07.2006, Uzun 963 (KATO: 18516); Ormanüstü village, afforestation site, 1683 m, 10.07.2008, Uzun 1390 (KATO: 18517); Çamlıdüz plateau, in forest, $1668 \mathrm{~m}$, 17.07.2008, Uzun 1615 (KATO: 18518);
Çamlıdüz plateau, in forest, $1720 \mathrm{~m}$, 17.07.2008, Uzun 1616 (KATO: 18519); Ormanüstü plateau, afforestation site, 1862 m, 02.08.2008, Uzun 1794 (KATO: 18520). Euro-Sib. el.

\section{Pilosella Hill.}

599. Pilosella hoppeana (Schult.) F.W. Schultz \& Sch.Bip. subsp. troica (Zahn) P.D.Sell \& C.West

Ormanüstü plateau, grassy place, $2020 \mathrm{~m}$, 05.08.2005, Uzun 833 (KATO: 18521); Çeşmeler district, stony hills, $634 \mathrm{~m}$, 07.07.2008, Uzun 1246 (KATO: 18522); Köprüyanı district, open forest, $701 \mathrm{~m}$, 08.07.2008, Uzun 1322 (KATO: 18523); ibid., $1001 \mathrm{~m}$, 08.07.2008, Uzun 1508 (KATO: 18524).

600. Pilosella hoppeana (Schult.) F.W. Schultz \& Sch.Bip. subsp. testimonialis (Nägeli ex Peter) P.D.Sell \& C.West Çatak, in forest, $1010 \mathrm{~m}, 14.07 .2008$, Uzun 1508 (KATO: 18525). Euro-Sib. el.

601. Pilosella ruprechtii (Boiss.) P.D.Sell \& C.West

Ormanüstü village, open forest, $1086 \mathrm{~m}$, 10.07.2008, Uzun 1391 (KATO: 18526).

602. Pilosella piloselloides (Vill.) Soják subsp. piloselloides

Çeşmeler district, stony hill, $583 \mathrm{~m}$, 07.07.2008, Uzun 1247 (KATO: 18528); Çeşmeler district, open place, $663 \mathrm{~m}$, 07.08.2008, Uzun 1840 (KATO: 18527).

603. Pilosella bauhini (Schult.) Arv.-Touv. subsp. magyarica (Peter) S.Bräut. (syn.: Pilosella piloselloides (Vill.) Soják subsp. magyarica (Peter) S.Bräut. \& Greuter, Pilosella piloselloides (Vill.) Soják subsp. megalomastix (NP.) P.D.Sell \& C.West) Ormanüstü village, open place, $950 \mathrm{~m}$, 08.07.2005, Uzun 740 (KATO: 18529); Çeşmeler district, stony hill, $490 \mathrm{~m}$, 07.07.2008, Uzun 1248 (KATO: 18531); Çeşmeler district, stony hill, $634 \mathrm{~m}$, 07.07.2008, Uzun 1249 (KATO: 18532); Sukenar1 district, in forest, $498 \mathrm{~m}$, 11.07.2008, Uzun 1434 (KATO: 18533).

604. Pilosella cymosa (L.) F.W.Schultz \& Sch.Bip. subsp. cymosa

Çeşmeler district, stony hill, $583 \mathrm{~m}$, 07.07.2008, Uzun 1250 (KATO: 18534); Çatak, in forest, $483 \mathrm{~m}, 14.07 .2008$, Uzun 1509 (KATO: 18535). Euro-Sib. el. 
605. Pilosella onegensis Norrl. (syn.: Pilosella caespitosa (Dumort.) P.D.Sell \& C.West subsp. brevipila (Nägeli \& Peter) P.D.Sell \& C.West)

Ormanüstü village, afforestation site, 1683 m, 10.07.2008, Uzun 1392 (KATO: 18536); Ormanüstü village, open place, $1879 \mathrm{~m}$, 20.07.2008, Uzun 1674 (KATO: 18537).

\section{Mycelis L.}

606. Mycelis muralis (L.) Dumort. Ormanüstü village, in forest, $1050 \mathrm{~m}$, 05.08.2005, Uzun 835 (KATO: 18542); Ormanüstü village, place filled with soil, 850 m, 26.07.2006, Uzun 964 (KATO: 18543). Euro-Sib. el.

\section{Lactuca L.}

607. Lactuca macrophylla (Willd.) A.Gray (syn.: Prenanthes cacaliifolia (M.Bieb.) Beauverd)

Ormanüstü village, in forest, $1530 \mathrm{~m}$, 05.08.2005, Uzun 834 (KATO: 18538). Eux. el.

608. Lactuca sativa L. subsp. serriola (L.) Galasso, Banfi, Bartolucci \& Ardenghi (syn.: Lactuca serriola L.) - Ac1 marul, Eşek marulu

Yukarıköy village, roadside, $931 \mathrm{~m}$, 14.07.2008, Uzun 1510 (KATO: 18540).

609. Lactuca tatarica (L.) CA.Mey. (syn.: Mulgedium tataricum (L.) DC.)

Ormanüstü village, in forest, $1680 \mathrm{~m}$, 08.07.2005, Uzun 741 (KATO: 18539).

610. Lactuca viminea (L.) J.Presl \& C.Presl subsp. viminea (syn.: Scariola viminea (L.) F.W.Schmidt)

Çeşmeler district, stony roadside, $437 \mathrm{~m}$, 07.07.2008, Uzun 1251 (KATO: 18541).

\section{Lapsana L.}

611. Lapsana grandiflora M.Bieb. (syn.: Lapsana communis L. subsp. grandiflora (M.Bieb.) P.D.Sell.)

Köprüyan1 district, in forest, $1001 \mathrm{~m}$, 08.07.2008, Uzun 1324 (KATO: 18544); Çatak, in forest, $483 \mathrm{~m}, 14.07 .2008$, Uzun 1511 (KATO: 18545); Ormanüstü plateau, afforestation site, $1862 \mathrm{~m}, 02.08 .2008$, Uzun 1795 (KATO: 18546); Çamlıdüz plateau, afforestation site, $1728 \mathrm{~m}, 20.07 .2008$, Uzun 1675 (KATO: 18547); ibid., $1786 \mathrm{~m}$, 20.07.2008, Uzun 1676 (KATO: 18548);
Ormanüstü plateau, Milia Tepesi, in forest, 1948 m, 22.07.2008, Uzun 1721 (KATO: 18549); Çamlıdüz plateau, in forest, $1740 \mathrm{~m}$, 22.07.2008, Uzun 1722 (KATO: 185509). Eux. (mt.) el.

\section{Taraxacum Wiggers - Karahindiba}

612. Taraxacum stevenii (Spreng.) DC. (syn.: Taraxacum crepidiforme DC.)

Ormanüstü plateau, grassy place, $1750 \mathrm{~m}$, 10.05.2008, Uzun 1050 (KATO: 18551). Ir.Tur. el.

613. Taraxacum scaturiginosum G.E.Haglund

Ormanüstü plateau, damp creekside, 1870, 05.06.2006, Uzun 929 (KATO: 18552).

614. Taraxacum buttleri Soest

Köprüyanı district, roadside slope, $580 \mathrm{~m}$, 10.05.2005, Uzun 600 (KATO: 18553).

\section{Crepis $\mathrm{L}$.}

615. Crepis setosa Haller $\mathrm{f}$.

Çeşmeler district, stony hill, $490 \mathrm{~m}$, 07.07.2008, Uzun 1252 (KATO: 18554).

Euro-Sib. el.

Dipsacales Juss. ex Bercht. \& J.Presl

F92 [64]. Adoxaceae E.Mey. (= Viburnaceae Raf.)

\section{Viburnum L. - Gilaburu, Kartopu}

616. Viburnum orientale Pall.

Köprüyanı district, forest edge, $1000 \mathrm{~m}$, 23.05.2006, Uzun 887 (KATO: 18396); Yenimahalle, in forest, $760 \mathrm{~m}, 26.07 .2006$, Uzun 957 (KATO: 18397). Eux. el. 617. Viburnum opulus L. - Kartopu Ormanüstü village, forest edge, $950 \mathrm{~m}$, 08.07.2005, Uzun 727 (KATO: 18398). Euro-Sib. el.

\section{Sambucus L. - Mürver}

618. Sambucus ebulus L. - Otsu mürver Ormanüstü village, forest edge, $1110 \mathrm{~m}$, 08.07.2005, Uzun 725 (KATO: 18394). Euro-Sib. el.

619. Sambucus nigra L. - Ağaç mürver Ormanüstü village, forest edge, $1680 \mathrm{~m}$, 08.07.2005, Uzun 726 (KATO: 18395). Euro-Sib. el. 
F93 [65]. Caprifoliaceae Juss. (including Dipsacaceae and Valerianaceae)

\section{Lonicera L. - Hanımeli \\ 620. Lonicera caucasica Pall. subsp. orientalis (Lam.) Chamb. \& Long - Kafkas hanımelisi \\ Ormanüstü village, forest edge, $950 \mathrm{~m}$, 08.07.2005, Uzun 723 (KATO: 18385); ibid., $1577 \mathrm{~m}$, 08.07.2005, Uzun 724 (KATO: 18386); Sukenar1 district, in forest, $663 \mathrm{~m}$, 07.07.2008, Uzun 1234 (KATO: 18387); Sukenarı district, in forest, $498 \mathrm{~m}$, 11.07.2008, Uzun 1431 (KATO: 18388); Çatak, in forest, $486 \mathrm{~m}, 14.07 .2008$, Uzun 1500 (KATO: 18389); Ormanüstü village, in forest, $1133 \mathrm{~m}$, 15.07.2008, Uzun 1557 (KATO: 18390). Endemic, IUCN: LC 621. Lonicera xylosteum $\mathrm{L}$. \\ Ormanüstü village, forest edge, $1200 \mathrm{~m}$, 05.08.2005, Uzun 818 (KATO: 18391); ibid., $1280 \mathrm{~m}, 09.07 .2008$, Uzun 1350 (KATO: 18392); ibid., $1086 \mathrm{~m}, 10.07 .2008$, Uzun 1386 (KATO: 18393).}

\section{Dipsacus L. -Tarak otu, Furça otu}

622. Dipsacus laciniatus L.

Yukarıköy village, road and field edges, 600 m, 29.07.2008, Uzun 1756 (KATO: 18404); Çatak, road and field edges, $870 \mathrm{~m}$, 30.07.2008, Uzun 1758 (KATO: 18405).

623. Dipsacus pilosus L.

Ormanüstü village, damp roadside, $1560 \mathrm{~m}$, 26.07.2006, Uzun 958 (KATO: 18406). Euro-Sib. el.

\section{Cephalaria Schrader ex Roemer \& Schultes - Acımık}

624. Cephalaria gigantea (Ledeb.) Bobrov Ormanüstü village, grassy place, $1400 \mathrm{~m}$, 12.07.2007, Uzun 1025 (KATO: 18407); ibid., 1280 m, 09.07.2008, Uzun 1351 (KATO: 18408). Eux. (mt.) el.

\section{Knautia L.}

625. Knautia involucrata Sommier \& Levier Ormanüstü village, grassy place, $1190 \mathrm{~m}$, 05.08.2005, Uzun 820 (KATO: 18409); Ormanüstü village, open place, $1620 \mathrm{~m}$, 05.08.2005, Uzun 821 (KATO: 18410); Yenimahalle, forest edge, $780 \mathrm{~m}, 26.07 .2006$, Uzun 959 (KATO: 18411); Ormanüstü plateau, roadside, $1750 \mathrm{~m}, 02.08 .2008$, Uzun 1790 (KATO: 18412). Eux. (mt.) el.

330. Scabiosa L. - Uyuz otu

626. Scabiosa columbaria L. subsp. columbaria

Çeşmeler district, stony hill, $583 \mathrm{~m}$, 07.07.2008, Uzun 1235 (KATO: 18413); Çatak, afforestation site, $477 \mathrm{~m}, 14.07 .2008$, Uzun 1501 (KATO: 18414).

627. Scabiosa intermedia (Post) Bornm. (bas.: Scabiosa columbaria L. subsp. columbaria var. intermedia (Post) Matthews) Çeşmeler district, roadside, $415 \mathrm{~m}$, 05.08.2005, Uzun 822 (KATO: 18415); Köprüyan1, open place, 700 m, 11.07.2007, Uzun 1009 (KATO: 18416); Kiremitli, roadside, 760-900 m, 13.07.2007, Uzun 1043 (KATO: 18417

\section{Valeriana L. - Kedi otu}

628. Valeriana alliariifolia Adams.

Ormanüstü village, forest edge, $950 \mathrm{~m}$, 08.07.2005, Uzun 728 (KATO: 18399); Ormanüstü village, damp hills, $1550 \mathrm{~m}$, 05.08.2005, Uzun 819 (KATO: 18400).

629. Valeriana leucophaea DC.

Ormanüstü plateau, stony place, $2000 \mathrm{~m}$, 05.06.2006, Uzun 927 (KATO: 18401). Hyr.Eux. (mt.) el.

332. Centranthus DC.

630. Centranthus longiflorus Steven subsp. longiflorus

Çatak, rocky slope, $400 \mathrm{~m}, 11.07 .2008$, Uzun 1432 (KATO: 18402). Ir.-Tur. el.

\section{Valerianella Mill.}

631. Valerianella dentata (L.) Pollich Çeşmeler district, stony hill, $880 \mathrm{~m}$, 18.06.2008, Uzun 1114 (KATO: 18403).

Apiales Nakai

F94 [66]. Araliaceae Juss.

\section{Hedera L. - Duvar sarmaşığı}

632. Hedera helix L. subsp. helix

Köprüyanı district, in forest, $1017 \mathrm{~m}$, 08.07.2008, Uzun 1303 (KATO: 18066). 633. Hedera colchica (K.Koch) K.Koch Ormanüstü village, in forest, $1104 \mathrm{~m}$, 09.07.2008, Uzun 1347 (KATO: 18067). Eux. el. 
F95 [67]. Apiaceae Lindl. (= Umbelliferae Juss.)

\section{Pastinaca L.}

634. Pastinaca sativa L. subsp. urens (Req. ex Godr.) Čelak.

Ormanüstü plateau, damp places in forest, 1596 m, 17.07.2008, Uzun 1604 (KATO: 18068).

\section{Sanicula $\mathbf{L}$.}

635. Sanicula europaea L.

Ormanüstü village, in forest, $950 \mathrm{~m}$, 08.07.2005, Uzun 685 (KATO: 18069).

Euro-Sib. el.

\section{Astrantia L.}

636. Astrantia maxima Pall. subsp. maxima Ormanüstü village, grassy place, $1000 \mathrm{~m}$, 10.05.2005, Uzun 584 (KATO: 18070); Ormanüstü village, forest edge, $929 \mathrm{~m}$, 08.07.2005, Uzun 686 (KATO: 18071). Eux. el.

637. Astrantia maxima Pall. subsp. haradjianii (I.Grinț.) Rech.f.

Ormanüstü village, damp places in forest, $1780 \mathrm{~m}$, 17.07.2008, Uzun 1605 (KATO: 18072); ibid., 1579 m, 20.07.2008, Uzun 1656 (KATO: 18073); Çamlıdüz plateau, afforestation site, $1728 \mathrm{~m}, 20.07 .2008$, Uzun 1657 (KATO: 18074). Endemic, IUCN: LC

\section{Eryngium L. - At dikeni}

638. Eryngium giganteum M.Bieb.

Çeşmeler district, open place, $440 \mathrm{~m}$, 08.07.2005, Uzun 687 (KATO: 18075); Çatak, open forest, $458 \mathrm{~m}, 14.07 .2008$, Uzun 1482 (KATO: 18076). Eux. el.

\section{Chaerophyllum L.}

639. Chaerophyllum aureum $\mathrm{L}$.

Ormanüstü village, forest edge, $1670 \mathrm{~m}$, 17.07.2008, Uzun 1606 (KATO: 18077).

\section{Anthriscus Pers.}

640. Anthriscus nemorosa (M.Bieb.) Spreng. Ormanüstü village, forest edge, $1670 \mathrm{~m}$, 17.07.2008, Uzun 1607 (KATO: 18078).

\section{Scaligeria DC.}

641. Scaligeria tripartita (Kalen.) Tamamsch.
Ormanüstü plateau, grassy place, $1850 \mathrm{~m}$, 05.06.2006, Uzun 915 (KATO: 18079). Eux. el.

342. Carum L.

642. Carum meifolium (M.Bieb.) Boiss.

Ormanüstü plateau, grassy place, $2000 \mathrm{~m}$, 05.06.2006, Uzun 916 (KATO: 18080); ibid., afforestation site, $1728 \mathrm{~m}, 20.07 .2008$, Uzun 1658 (KATO: 18081).

\section{Pimpinella L.}

643. Pimpinella rhodantha Boiss.

Ormanüstü village, grassy place, $950 \mathrm{~m}$, 26.07.2006, Uzun 947 (KATO: 18082); ibid., forest edge, $1445 \mathrm{~m}, 17.07 .2008$, Uzun 1608 (KATO: 18083); Çamlıdüz plateau, afforestation site, $1728 \mathrm{~m}, 20.07 .2008$, Uzun 1659 (KATO: 18084).

644. Pimpinella tragium Vill. subsp. polyclada (Boiss. \& Heldr.) Tutin

Çeşmeler district, stony hills, $634 \mathrm{~m}$, 07.07.2008, Uzun 1189 (KATO: 18085); Çeşmeler district, stony hill, $632 \mathrm{~m}$, 11.07.2008, Uzun 1425 (KATO: 18086); Sukenar1 district, in forest, $498 \mathrm{~m}$, 11.07.2008, Uzun 1426 (KATO: 18087); Çatak, open forest, $458 \mathrm{~m}, 14.07 .2008$, Uzun 1483 (KATO: 18088).

\section{Seseli L.}

645. Seseli petraeum M.Bieb.

Çeşmeler district, rocky place, $890 \mathrm{~m}$, 18.06.2008, Uzun 1104 (KATO: 18089); Çeşmeler district, stony hills, $490 \mathrm{~m}$, 07.07.2008, Uzun 1190 (KATO: 18090); Kiremitli district, on rocks, $760 \mathrm{~m}$, 13.07.2007, Uzun 1038 (KATO: 18091). Eux. (mt.) el., Rare, IUCN: VU

\section{Aethusa L.}

646. Aethusa cynapium L. subsp. cynapium Köprüyan1 district, open forest, $975 \mathrm{~m}$, 08.07.2008, Uzun 1304 (KATO: 18092). Euro-Sib. el.

\section{Foeniculum Mill.}

647. Foeniculum vulgare Mill. subsp. vulgare - Rezene

Çatak district, roadside slopes, $490 \mathrm{~m}$, 07.07.2008, Uzun 1191 (KATO: 18093). 


\section{Physospermum Cusson}

648. Physospermum cornubiense (L.) DC. Çeşmeler district, stony hills, $634 \mathrm{~m}$, 07.07.2008, Uzun 1192 (KATO: 18094); Çeşmeler district, in forest, $529 \mathrm{~m}$, 14.07.2008, Uzun 1484 (KATO: 18095); Çamlıdüz plateau, afforestation site, $1728 \mathrm{~m}$, 20.07.2008, Uzun 1660 (KATO: 18096); Çamlıdüz plateau, in forest, $1786 \mathrm{~m}$, 20.07.2008, Uzun 1661 (KATO: 18097); Çamlıdüz plateau, open forest, $1892 \mathrm{~m}$, 20.07.2008, Uzun 1662 (KATO: 18098); Çamlıdüz plateau, in forest, $1740 \mathrm{~m}$, 22.07.2008, Uzun 1715 (KATO: 18099).

\section{Bupleurum L.}

649. Bupleurum falcatum L. subsp. persicum (Boiss.) Koso-Pol.

Çatak, afforestation site, $873 \mathrm{~m}, 06.08 .2008$, Uzun 1828 (KATO: 18100).

\section{Peucedanum L.}

650. Peucedanum longifolium Waldst. \& Kit Çeşmeler district, edge of rocks, $1050 \mathrm{~m}$, 11.07.2007, Uzun 993 (KATO: 18101). Euro-Sib. el.

\section{Heracleum $\mathbf{L}$.}

651. Heracleum platytaenium Boiss. Tavşancil otu

Çeşmeler district, grassy place, $1050 \mathrm{~m}$, 11.07.2007, Uzun 994 (KATO: 18102); Vicinity of Vazelon monastry, open place, 1200 m, 24.07.2008, Uzun 1740 (KATO: 18103). Eux. el., Endemic, IUCN: LC

\section{Laser Borkh.}

652. Laser trilobum (L.) Borkh. - Kefe kimyonu

Çeşmeler district, forest edge, rocky place, $550 \mathrm{~m}$, 01.06.2008, Uzun 1069 (KATO: 18104).

*653. Laser affine (Ledeb.) Wojew. \& Spalik (bas.: Laserpitium affine Ledeb.)

Çamlıdüz plateau, in forest, $1769 \mathrm{~m}$, 22.07.2008, Uzun 1716 (KATO: 18105). Eux. (mt.) el., Rare, IUCN: DD

\section{Torilis Adans.}

654. Torilis arvensis (Huds.) Link subsp. arvensis

Çeşmeler district, stony place, $490 \mathrm{~m}$, 07.07.2008, Uzun 1193 (KATO: 18106).

\section{Caucalis $\mathbf{L}$.}

655. Caucalis platycarpos $\mathrm{L}$.

Çeşmeler district, stony hill, $490 \mathrm{~m}$, 07.07.2008, Uzun 1194 (KATO: 18107).

\section{Daucus L.}

656. Daucus carota L. subsp. carota Yabani havuç

Yukarıköy village, grassy place, $931 \mathrm{~m}$, 14.07.2008, Uzun 1485 (KATO: 18108). Cultivated 